\title{
Characteristic initial data and smoothness of Scri. II. Asymptotic expansions and construction of conformally smooth data sets*
}

\author{
Tim-Torben Paetz ${ }^{\dagger}$ \\ Gravitational Physics, University of Vienna \\ Boltzmanngasse 5, 1090 Vienna, Austria
}

June 26, 2018

\begin{abstract}
We derive necessary-and-sufficient conditions on characteristic initial data for Friedrich's conformal field equations in $3+1$ dimensions to have no logarithmic terms in an asymptotic expansion at null infinity.
\end{abstract}

PACS: 02.30.Hq, 02.30.Mv, 04.20.Ex, 04.20.Ha

\section{Contents}

1 Introduction 2

2 Preliminaries 3

2.1 Notation . . . . . . . . . . . . . . . . . . . . 3

2.2 Setting . . . . . . . . . . . . . . . .

2.3 A priori restrictions . . . . . . . . . . . . . 6

3 Asymptotic solutions of Einstein's characteristic vacuum constraint equations

3.1 Asymptotic solutions of the constraint equations . . . . . . . . .

3.1.1 Expansion of $\varphi \ldots \ldots \ldots \ldots \ldots \ldots$

3.1 .2 Expansion of $\nu^{0} \ldots \ldots \ldots \ldots \ldots \ldots$

3.1 .3 Expansion of $\xi_{A} \ldots \ldots \ldots \ldots$. . . . . . . . . . . 11

3.1.4 Expansion of $\nu_{A} \ldots \ldots \ldots \ldots \ldots \ldots$

3.1.5 Expansion of $\zeta \ldots \ldots \ldots \ldots \ldots \ldots \ldots$

3.1 .6 Expansion of $\bar{g}^{r r} \ldots \ldots \ldots \ldots \ldots \ldots$

3.2 The no-logs-condition . . . . . . . . . . . . . . . 15

3.3 Summary and discussion . . . . . . . . . . . . . . . . 16

*Preprint UWThPh-2014-9.

${ }^{\dagger}$ Email Tim-Torben.Paetz@univie.ac.at 
4 Metric gauge $\mathbf{1 8}$

4.1 Introduction . . . . . . . . . . . . . . . . . 18

4.2 Gauge scheme . . . . . . . . . . . . . . . . . 19

4.3 Metric gauge and some conventions . . . . . . . . . . . . . 24

4.4 Solution of the constraint equations in the metric gauge . . . . . 24

4.5 Overview of the metric gauge I . . . . . . . . . . . . . 26

4.6 Transverse derivatives of the metric on $\mathscr{N} \ldots \ldots . . . . . . .27$

4.7 Overview of the metric gauge II . . . . . . . . . . . . . . 33

5 Asymptotic Expansions of the unknowns of the CFE on $\mathscr{N}$

5.1 Conformal field equations $(\mathrm{CFE}) \ldots \ldots \ldots 34$

5.1.1 Metric conformal field equations (MCFE) . . . . . . . . . 34

5.1.2 General conformal field equations (GCFE) . . . . . . . . 35

5.2 Asymptotic behavior of the fields appearing in the CFE . . . . . 35

5.2.1 Asymptotic behavior of the metric tensor . . . . . . . . . 36

5.2.2 Asymptotic behavior of the Weyl tensor . . . . . . . . . . 36

5.2.3 Asymptotic behavior of $\overline{\partial_{0} \Theta}$ and $\overline{\partial_{00}^{2} \Theta} \ldots$. . . . . . . . . 36

5.2.4 Asymptotic behavior of the Christoffel symbols . . . . . . 37

5.2.5 Asymptotic behavior of the Schouten tensor . . . . . . . . 38

5.2.6 Asymptotic behavior of the function $\tilde{s}$. . . . . . . . . 38

5.3 Main result ...................... 39

A Asymptotic solutions of Fuchsian ODEs 40

A.1 Formal solutions . . . . . . . . . . . . . . . . . . . 40

A.1.1 Scalar equation $x \partial_{x} f+h f=g \ldots \ldots . \ldots . \ldots 40$

A.1.2 ODE-system $x \partial_{x} f+h f=g \ldots \ldots . \ldots . \ldots 42$

A.2 Borel summation . . . . . . . . . . . . . . . . . . . . 45

A.3 Approximation of the exact solution ........... 45

B Relation between $\kappa=0$ - and $\kappa=\frac{r}{2}|\sigma|^{2}$-gauge

\section{Introduction}

In this work we continue the work initiated in 7] to analyze the occurrence of logarithmic terms in the asymptotic expansion of the metric tensor and some other fields at null infinity. In part I of this work, where we also described the setting, it has been shown that the harmonic coordinate condition is not compatible with a smooth asymptotic structure at the conformal boundary at infinity, but has to be replaced by a wave-map gauge condition with non-vanishing gauge-source functions.

However, it is expected 5, 12] (compare also [1]) that even for smooth initial data the asymptotic expansion of the space-time metric at null infinity will generically be polyhomogeneous and involve logarithmic terms which do not have their origin in an inconvenient choice of coordinates. One main object of this note, treated in Section 3, is to study thoroughly the asymptotic behavior of solutions of the Einstein's vacuum constraint equations and analyze under which conditions a smooth conformal completion of the restriction of the spacetime metric to the characteristic initial surface across null infinity is possible. As announced in [7, Theorem 5.1] we intend to provide necessary-and-sufficient 
conditions on the initial data and the gauge source functions which permit such extensions. In doing so it will become manifest that many, though not all, of the logarithmic terms which arise at infinity are gauge artifacts. The remaining non-gauge logarithmic terms can be eliminated by imposing restrictions on the asymptotic behavior of the initial data, captured by what we call no-logscondition.

In Section [5, we will show that solutions of the characteristic vacuum constraint equations satisfying the no-logs-condition lead to smooth initial data for Friedrich's conformal field equations. The data will be computed in a new gauge scheme developed in Section 4 and will provide the basis to solve the evolution problem and construct space-times with a "piece of smooth $\mathscr{I}^{+}$".

In Section 2 we give a summary of 7] where we briefly describe the framework and recall the most important definitions and results of part I. Finally, in Appendix A our proceeding in Section 3/5 to solve the constraint equations in terms of polyhomogeneous expansions will be rigorously justified, while in Appendix B we compare the peculiarities of different gauge schemes.

\section{Preliminaries}

We use all the notation, terminology and conventions introduced in part I 7]. For the convenience of the reader, though, let us briefly recall the most essential ingredients and definitions of our framework.

\section{$2.1 \quad$ Notation}

Consider a smooth function

$$
f:(0, \infty) \times S^{2} \longrightarrow \mathbb{R}, \quad\left(r, x^{A}\right) \longmapsto f\left(r, x^{A}\right) .
$$

If this function permits an asymptotic expansion as a power series in $r$, we denote by $f_{n}$, or $(f)_{n}$, the coefficient of $r^{-n}$ in the corresponding expansion. Be aware that sometimes a lower index might denote both the component of a vector, and the $n$-th order term in an expansion of the corresponding object. If both indices need to appear simultaneously we use brackets and place the index corresponding to the $n$-th order expansion term outside the brackets. We write

$$
f\left(r, x^{A}\right) \sim \sum_{k=-N}^{\infty} f_{k}\left(x^{A}\right) r^{-k}
$$

if the right-hand side is the polyhomogeneous expansion at $x=0$ of the function $\left.x \mapsto r^{-N} f(r, \cdot)\right|_{r=1 / x}$. Moreover, we write $f=\mathcal{O}\left(r^{N}\right)\left(\right.$ or $f=\mathcal{O}\left(x^{-N}\right), x \equiv$ $1 / r), N \in \mathbb{Z}$, if the function $\left.x \mapsto r^{-N} f(r, \cdot)\right|_{r=1 / x}$ is smooth at $x=0$.

\subsection{Setting}

We consider a $3+1$-dimensional $C^{\infty}$-manifold $\mathscr{M}$. For definiteness we take as initial surface either a globally smooth light-cone $C_{O} \subset \mathscr{M}$ or two null hypersurfaces $\mathscr{N}_{1}, \mathscr{N}_{2} \subset \mathscr{M}$ intersecting transversally along a smooth submanifold $S \cong S^{2}$. Suppose that the closure (in the completed space-time) $\overline{\mathscr{N}}$ of $\mathscr{N} \in$ $\left\{C_{O} \backslash\{O\}, \mathscr{N}_{1}\right\}$ meets $\mathscr{I}^{+}$transversally in a smooth spherical cross-section. 
We introduce adapted null coordinates $\left(u \equiv x^{0}, r \equiv x^{1}, x^{A}\right)$ on $\mathscr{N}$ (i.e. $\mathscr{N}=\{u=0\}$, where $r$ parameterizes the null rays generating $\mathscr{N}$, and $\left(x^{A}\right)$ are local coordinates on $\Sigma_{r} \equiv\{r=$ const, $\left.u=0\} \cong S^{2}\right)$. Then the trace $\bar{g}$ of the metric on $\mathscr{N}$ becomes

$$
\bar{g}=\bar{g}_{00} \mathrm{~d} u^{2}+2 \nu_{0} \mathrm{~d} u \mathrm{~d} r+2 \nu_{A} \mathrm{~d} u \mathrm{~d} x^{A}+\check{g},
$$

where

$$
\check{g}=\check{g}_{A B} \mathrm{~d} x^{A} \mathrm{~d} x^{B}:=\bar{g}_{A B} \mathrm{~d} x^{A} \mathrm{~d} x^{B}
$$

is a degenerate quadratic form induced by $g$ on $\mathscr{N}$ which induces on each slice $\Sigma_{r}$ an $r$-dependent Riemannian metric $\check{g}_{\Sigma_{r}}$ (coinciding with $\check{g}(r, \cdot)$ in the coordinates above). While the components $\bar{g}_{00}, \nu_{0}$ and $\nu_{A}$ depend upon the choice of coordinates off $\mathscr{N}$, the quadratic form $\check{g}$ is intrinsically defined on $\mathscr{N}$.

In fact, we will be interested merely in the asymptotic behavior of the restriction of the space-time metric to $\mathscr{N}$. A regular light-cone or two transversally intersecting null hypersurfaces with appropriately specified initial data, though, guarantee that the vacuum constraint equations have unique solutions.

Throughout this work we use an overline to denote a space-time object restricted to $\mathscr{N}$. The symbol " $"$ " will be used to denote objects associated with the Riemannian metric $\check{g}_{\Sigma_{r}}$.

Definition 2.1 (cf. 7]) We say that a smooth metric tensor $\bar{g}_{\mu \nu}$ defined on a null hypersurface $\mathscr{N}$ given in adapted null coordinates has a smooth conformal completion at infinity if the unphysical metric tensor $\overline{\tilde{g}}_{\mu \nu}$ obtained via the coordinate transformation $r \mapsto 1 / r=: x$ and the conformal rescaling $\bar{g} \mapsto \overline{\tilde{g}} \equiv x^{2} \bar{g}$ is, as a Lorentzian metric, smoothly extendable at $\{x=0\}$.

The components of a smooth tensor field on $\mathscr{N}$ will be said to be smooth at infinity whenever they admit a smooth expansion in the conformally rescaled space-time at $\{x=0\}$ and expressed in the $\left(x, x^{A}\right)$-coordinates.

As remarked in [7], Definition 2.1 concerns only fields on $\mathscr{N}$ and is not tied to the existence of an associated space-time. Moreover, it concerns both conditions on the metric and on the coordinate system.

The Einstein equations split into a set of evolution equations and a set of constraint equations which need to be satisfied on the initial surface. In the characteristic case the data for the evolution equations are provided by the trace of the metric on the initial surface. Due to the constraints not all of its components can be prescribed freely. There are various ways of choosing free data [6, 7]. Here we focus on Rendall's scheme [10], where the free data are formed by the conformal class $[\gamma]$ of the tensor $\check{g}_{\Sigma_{r}}$ (and the function $\kappa$ introduced below) 11 By choosing a representative they can be viewed as a oneparameter family, parameterized by $r$, of Riemannian metrics $\gamma(r, \cdot)$ on $S^{2}$. A major advantage of this scheme in particular in view of Section 4 is that it permits a separation of physical and gauge degrees of freedom. Some comments on how things change for other approaches to prescribe characteristic initial data are given in [7], cf. Remark 3.4

Einstein's vacuum constraint equations in a $\hat{g}$-generalized wave-map gauge are obtained from Einstein's vacuum equations assuming that the wave-gauge vector

$$
H^{\lambda}:=\Gamma^{\lambda}-\hat{\Gamma}^{\lambda}-W^{\lambda}=0, \quad \Gamma^{\lambda}:=g^{\alpha \beta} \Gamma_{\alpha \beta}^{\lambda}, \quad \hat{\Gamma}^{\lambda}:=g^{\alpha \beta} \hat{\Gamma}_{\alpha \beta}^{\lambda} .
$$

\footnotetext{
${ }^{1}$ In the case of two transversally intersecting null hypersurfaces these data need to be supplemented by corresponding data on $\mathscr{N}_{2}$ and certain data on the intersection manifold $S$.
} 
vanishes. We use the hat-symbol "^" to indicate objects associated with some target metric $\hat{g}$, which we assume for convenience to be of the form

$$
\begin{gathered}
\overline{\hat{g}}_{1 i}=0, \quad \overline{\hat{g}}_{A B}=r^{2} s_{A B}+\mathcal{O}(1), \quad \hat{\nu}_{0}=1+\mathcal{O}\left(r^{-3}\right), \quad \hat{\nu}_{A}=\mathcal{O}\left(r^{-2}\right) \\
\overline{\hat{g}}_{00}=-1+\mathcal{O}\left(r^{-2}\right), \quad \overline{\partial_{0} \hat{g}_{1 i}}=\mathcal{O}\left(r^{-3}\right), \quad \bar{\partial}_{0} \hat{g}_{A B}=\mathcal{O}\left(r^{-1}\right)
\end{gathered}
$$

on $\mathscr{N}$, where $s=s_{A B} \mathrm{~d} x^{A} \mathrm{~d} x^{B}$ is the unit round metric on the sphere $S^{2}$. By $W^{\lambda}=W^{\lambda}\left(x^{\mu}, g_{\mu \nu}\right)$ we denote the components of a vector field, the gauge source functions, which can be arbitrarily prescribed. They reflect the freedom to choose coordinates off the initial surface, and thus allow us to analyze smoothness of the metric tensor at infinity in arbitrary coordinates.

For given initial data $\gamma=\gamma_{A B} \mathrm{~d} x^{A} \mathrm{~d} x^{B}$ the wave-map gauge constraints form a hierarchical system of ODEs along the null generators of the cone (cf. [2]):

$$
\begin{aligned}
\partial_{r r}^{2} \varphi-\kappa \partial_{r} \varphi+\frac{1}{2}|\sigma|^{2} \varphi & =0 \\
\left(\partial_{r}+\frac{1}{2} \tau+\kappa\right) \nu^{0}+\frac{1}{2}\left(\bar{W}^{0}+\overline{\hat{\Gamma}}^{0}\right) & =0 \\
\left(\partial_{r}+\tau\right) \xi_{A}-2 \check{\nabla}_{B} \sigma_{A}^{B}+\partial_{A} \tau+2 \partial_{A} \kappa & =0 \\
2 \nu^{0}\left(\partial_{r} \nu_{A}-2 \nu_{B} \chi_{A}{ }^{B}\right)-\nu_{A}\left(\bar{W}^{0}+\overline{\hat{\Gamma}}^{0}\right)-\bar{g}_{A B}\left(\bar{W}^{B}+\overline{\hat{\Gamma}}^{B}\right) & \\
+\gamma_{A B} \gamma^{C D} \check{\Gamma}_{C D}^{B}+\xi_{A} & =0 \\
\left(\partial_{r}+\tau+\kappa\right) \zeta+\check{R}-\frac{1}{2} \xi_{A} \xi^{A}+\check{\nabla}_{A} \xi^{A} & =0 \\
\left(2 \partial_{r}+\tau+2 \kappa\right) \bar{g}^{r r}+2 \bar{W}^{r}+2 \overline{\hat{\Gamma}}^{r}-\zeta & =0
\end{aligned}
$$

where $\tau$ and $\sigma_{A}{ }^{B}$ denote the expansion and the shear of $\mathscr{N}$, respectively,

$$
\kappa=\bar{\Gamma}_{r r}^{r}, \quad \xi_{A}=-2 \bar{\Gamma}_{r A}^{r}, \quad \zeta=2 \bar{g}^{A B} \bar{\Gamma}_{A B}^{r}+\tau \bar{g}^{r r} .
$$

Apart from $W^{\lambda}$ the function $\kappa$ turns out to be another gauge degree of freedom, reflecting the freedom to choose $r$, which can be prescribed conveniently.

Integrating these equations successively one determines all the components of $\bar{g}$ (note that $\nu_{0}=\left(\nu^{0}\right)^{-1}$ and $\bar{g}_{00}=\bar{g}^{A B} \nu_{A} \nu_{B}-\left(\nu_{0}\right)^{2} \bar{g}^{r r}$ ). The relevant boundary conditions follow either from regularity conditions at the vertex [2] when $\mathscr{N}$ is a cone, or from the remaining data specified on $\mathscr{N}_{2}$ and $S$ (cf. e.g. [6, 10]) in the case of two characteristic surfaces intersecting transversally.

Our ultimate goal is to find necessary-and-sufficient conditions on the initial data given on $\mathscr{N}$, such that the resulting space-time has a smooth conformal completion at infinity à la Penrose. This requires the following ingredients:

1. To exclude the appearance of conjugate points or coordinate singularities on $\mathscr{N}$, the constraint equations need to admit a non-degenerated global solution $\bar{g}$ on $\mathscr{N}$. This will be the case if and only if the functions $\varphi$ and $\nu^{0}$ are of constant sign,

$$
\varphi \neq 0, \quad \nu^{0} \neq 0 \quad \text { on } \mathscr{N} .
$$

2. The metric $\bar{g}$ needs to be smoothly extendable as a Lorentzian metric, which means that the functions $\varphi_{-1}$ and $\left(\nu^{0}\right)_{0}$ need to have a sign

$$
\varphi_{-1} \neq 0, \quad\left(\nu^{0}\right)_{0} \neq 0 \quad \text { on } S^{2} .
$$

This assumption excludes conjugate points at the intersection of $\mathscr{N}$ with $\mathscr{I}^{+}$. 
3. The components of $\bar{g}$ need to be smooth at $\mathscr{I}^{+}$. For this one has to make sure that their asymptotic expansions contain no logarithmic terms and have the correct order in terms of powers of $r$.

4. All the fields which appear in Friedrich's conformal field equations (which provide an evolution system which, in contrast to Einstein's field equations, is well-behaved at $\mathscr{I}^{+}$) need to be smooth at $\mathscr{I}^{+}$.

5. Finally, an appropriate well-posedness result for the conformal field equations is needed.

Point 1 and 2 have been addressed in [7], cf. Proposition 2.2 below. Point 3 will be the subject of Section 3 , while point 4 will be investigated in Section 5 . Point 5 will be addressed elsewhere.

From now on we shall consider exclusively conformal data $\gamma_{A B}(r, \cdot) d x^{A} d x^{B}$ and gauge functions $\kappa$ and $\bar{W}^{\lambda}$ for which (2.12) and (2.13) hold. Let us summarize some of the results established in [7] (adapted to the smooth setting on which we focus here) which provide sufficient conditions such that 2.12) and (2.13) hold in the case where $\mathscr{N}$ represents a regular light-cone $C_{O} \sqrt{2}$

Proposition 2.2 1. Solutions of the Raychaudhuri equation (2.5) with prescribed $\kappa=\mathcal{O}\left(r^{-3}\right)$ and $\sigma_{A}{ }^{B}=\mathcal{O}\left(r^{-2}\right)$ lead to a globally positive $\varphi$ on $C_{O} \backslash\{O\}$ with $\varphi_{-1}>0$ on $S^{2}$ if

$\int_{0}^{\infty}\left(\int_{0}^{r} e^{H(\hat{r})} \mathrm{d} \hat{r}\right) e^{-H(r)}|\sigma|^{2}(r) \mathrm{d} r<2$, where $H\left(r, x^{A}\right):=\int_{0}^{r} \kappa\left(\tilde{r}, x^{A}\right) d \tilde{r}$.

2. Assuming a Minkowski target and

$\bar{W}^{0}=\mathcal{O}\left(r^{-1}\right)$ with $\bar{W}^{0}<r \varphi^{-2} \sqrt{\frac{\operatorname{det} \gamma}{\operatorname{det} s}} \gamma^{A B} s_{A B}$ and $\left(\bar{W}^{0}\right)_{1}<2\left(\varphi_{-1}\right)^{-2}$,

any positive solution $\varphi$ of (2.5) leads to a globally defined positive function $\nu_{0}$ on $C_{O}$ with $0<\left(\nu_{0}\right)_{0}<\infty$.

\section{$2.3 \quad$ A priori restrictions}

Before we analyze thoroughly the asymptotic behavior of the vacuum Einstein constraint equations and derive necessary-and-sufficient conditions concerning smoothness of the solutions at infinity it is convenient to have some a priori knowledge regarding the lowest admissible orders of the gauge functions, and to exclude the appearance of log terms in the expansion of "auxiliary" fields such as $\xi_{A}=\bar{\Gamma}_{r A}^{r}$. In 7] we have shown that the following equations need to be necessarily fulfilled in some adapted null coordinate system to end up with a trace of a metric on $\mathscr{N}$ which admits a smooth conformal completion and infinity, and connection coefficients which are smooth at $\mathscr{I}^{+}$:

$$
\kappa=\mathcal{O}\left(r^{-3}\right), \quad \bar{W}^{0}=\mathcal{O}\left(r^{-1}\right), \quad \xi_{A}=\mathcal{O}\left(r^{-1}\right), \quad \bar{W}^{A}=\mathcal{O}\left(r^{-1}\right) .
$$

Moreover, we may assume the initial data to be of the asymptotic form

$$
\gamma_{A B} \sim r^{2}\left(s_{A B}+\sum_{n=1}^{\infty} h_{A B}^{(n)} r^{-n}\right)
$$

\footnotetext{
${ }^{2}$ In the conventions of 7 ] the functions $\varphi, \nu^{0}, \varphi_{-1}$ and $\left(\nu^{0}\right)_{0}$ need to be positive.
} 
for some smooth tensor fields $h_{A B}^{(n)}$ on $S^{2}$. If $\gamma$ is not of the form (2.15), it can either be brought to it via a conformal rescaling and an suitable choice of $r$, or it leads to a metric $\bar{g}$ which does not have a smooth conformal completion at $\mathscr{I}^{+}$.

At this stage we do not know whether a space-time which admits a conformal completion at infinity is compatible with polyhomogeneous rather than smooth expansions of the functions $\zeta$ and $\bar{W}^{r}$. It will turn out that this is not the case. However, we note that it follows from the constraint equations and the above a priori restrictions that

$$
\text { if } \zeta=\mathcal{O}\left(r^{-1}\right) \text { then } \bar{W}^{r}=\mathcal{O}(r) \text {. }
$$

\section{Asymptotic solutions of Einstein's character- istic vacuum constraint equations}

It is useful to introduce some notation first: Let $w_{A B}$ be a rank-2-tensor on the initial surface $\mathscr{N}$. We denote by $\breve{w}_{A B}$, or $\left(w_{A B}\right)^{\zeta}$, its trace-free part w.r.t. the metric $\bar{g}_{A B} \mathrm{~d} x^{A} \mathrm{~d} x^{B}$. Consider now the expansion coefficients $\left(w_{A B}\right)_{n}$ at infinity, which are tensors on $S^{2}$. We denote by $\left(\breve{w}_{A B}\right)_{n}$, or $\left(w_{A B}\right)_{n}$, the trace-free part w.r.t. the unit round metric $s=s_{A B} \mathrm{~d} x^{A} \mathrm{~d} x^{B}$. Finally, we set

$$
|w|^{2}:=\bar{g}^{A C} \bar{g}^{B D} w_{A B} w_{C D}, \quad \text { and } \quad\left|w_{n}\right|^{2}:=s^{A C} s^{B D}\left(w_{A B}\right)_{n}\left(w_{C D}\right)_{n} .
$$

Let us make the convention to raise indices of the expansion coefficients $h_{A B}^{(n)}$ with the standard metric. Moreover, we set

$$
h^{(n)}:=s^{A B} h_{A B}^{(n)} .
$$

A ring ${ }^{\circ}$ on a covariant derivative operator or a connection coefficient indicates that the corresponding object is associated to $s=s_{A B} \mathrm{~d} x^{A} \mathrm{~d} x^{B}$.

\subsection{Asymptotic solutions of the constraint equations}

The object of this section is twofold: First of all we will show that the Einstein wave-map gauge constraints (2.5)-(2.10) can be solved asymptotically in terms of polyhomogeneous expansions at infinity of the solution. This is done by rewriting the equations in a form to which Appendix $\mathrm{A}$ applies. The second aim is to make some general considerations concerning the appearance of logarithmic terms in the asymptotic solutions of (2.5)-(2.10) for initial data of the form (2.15). We want to extract necessary-and-sufficient conditions leading to the trace $\bar{g}$ of a metric on $\mathscr{N}$ which admits a smooth conformal completion at infinity in the sense of Definition 2.1.

Our starting point are initial data $\gamma$ with an asymptotic behavior of the form (2.15) and gauge functions

$$
\kappa=\mathcal{O}\left(r^{-3}\right), \quad \bar{W}^{0}=\mathcal{O}\left(r^{-1}\right), \quad \bar{W}^{A}=\mathcal{O}\left(r^{-1}\right), \quad \bar{W}^{r}=\mathcal{O}(r),
$$

for which $\varphi, \nu^{0}, \varphi_{-1}$ and $\left(\nu^{0}\right)_{0}$ have a sign on $\mathscr{N}$ and $S^{2}$, respectively. We further require that the asymptotic expansion of $\xi_{A}$ contains no logarithmic terms, i.e. $\xi_{A}=\mathcal{O}\left(r^{-1}\right)$. A violation of one of these assumptions would not be compatible with a space-time which admits a smooth conformal completion at infinity 
as follows from the a priori restriction and the following fact: The considerations below reveal that (2.15),$\kappa=\mathcal{O}\left(r^{-3}\right)$ and $\xi_{A}=\mathcal{O}\left(r^{-1}\right)$ imply $\zeta=\mathcal{O}\left(r^{-1}\right)$, and that (2.16) applies. So we are imposing no restrictions when assuming $\bar{W}^{r}=\mathcal{O}(r)$.

Consider the shear tensor,

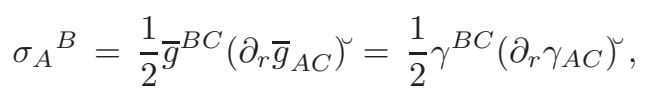

whose asymptotic expansion we express in terms of the expansion coefficients of the initial data $\gamma$

$$
\begin{aligned}
\sigma_{A}{ }^{B} & =-\frac{1}{2} \breve{h}_{A}^{(1) B} r^{-2}+\left(\frac{1}{2} h^{(1)} \breve{h}_{A}^{(1) B}-\breve{h}_{A}^{(2) B}\right) r^{-3}+\mathcal{O}\left(r^{-4}\right), \\
|\sigma|^{2} & =\frac{1}{4}\left|\breve{h}^{(1)}\right|^{2} r^{-4}+\left(\breve{h}_{A}^{(1) B} \breve{h}_{B}^{(2) A}-\frac{1}{2} h^{(1)}\left|\breve{h}^{(1)}\right|^{2}\right) r^{-5}+\mathcal{O}\left(r^{-6}\right) .
\end{aligned}
$$

A global solution, and thereby also the value of the "asymptotic integration functions", to each of the constraint ODEs is determined by regularity conditions at the vertex $O$ of a light-cone, or by the data on the intersection manifold $S$ for two intersecting characteristic surfaces. However, the integration functions, which depend on the initial data $\gamma$, the gauge functions and the boundary conditions at the vertex or the intersection manifold, appear difficult to control.

\subsubsection{Expansion of $\varphi$}

We start with the constraint equation (2.5) for the function $\varphi$,

$$
\partial_{r r}^{2} \varphi-\kappa \partial_{r} \varphi+\frac{1}{2}|\sigma|^{2} \varphi=0 .
$$

In order to enable an easier comparison to Appendix $\AA$ we make the transformation $r \mapsto 1 / r \equiv x$, with $\varphi, \kappa$ and $|\sigma|^{2}$ treated as scalars, and rewrite the ODE as a first-order system. The equation then reads with $\varphi^{(1)}:=\varphi$ and $\varphi^{(2)}:=x \partial_{x} \varphi$,

$$
\left[x \partial_{x}+\left(\begin{array}{cc}
0 & -1 \\
\frac{|\sigma|^{2}}{2 x} & 1+\frac{\kappa}{x}
\end{array}\right)\right]\left(\begin{array}{l}
\varphi^{(1)} \\
\varphi^{(2)}
\end{array}\right)=0,
$$

or, when the leading order term is diagonalized,

$$
[x \partial_{x}+\left(\begin{array}{ll}
1 & 0 \\
0 & 0
\end{array}\right)+\underbrace{\left.\left(\begin{array}{cc}
\frac{\kappa}{x}-\frac{|\sigma|^{2}}{2 x} & -\frac{|\sigma|^{2}}{\sqrt{2} x} \\
\frac{|\sigma|^{2}}{2^{3 / 2} x}-\frac{\kappa}{\sqrt{2} x} & \frac{|\sigma|^{2}}{2 x}
\end{array}\right)\right]}_{=\mathcal{O}(x)}]\left(\begin{array}{l}
\tilde{\varphi}^{(1)} \\
\tilde{\varphi}^{(2)}
\end{array}\right)=0,
$$

with

$$
\left(\begin{array}{l}
\tilde{\varphi}^{(1)} \\
\tilde{\varphi}^{(2)}
\end{array}\right):=\left(\begin{array}{cc}
0 & -\sqrt{2} \\
1 & 1
\end{array}\right)\left(\begin{array}{l}
\varphi^{(1)} \\
\varphi^{(2)}
\end{array}\right) .
$$

The results in Appendix $\mathrm{A}$ (we have, in the notation used there, $\lambda_{1}=-1$, $\lambda_{2}=0$, and, since there is no source, $\hat{\ell}=-1$ ) shows that this ODE can be 
solved asymptotically via a polyhomogeneous expansion with $\tilde{\varphi}^{(n)}=O\left(x^{-1}\right)$. It also reveals that the coefficients $\left(\tilde{\varphi}^{(n)}\right)_{\lambda_{n}}, n=1,2$, i.e. $\left(\tilde{\varphi}^{(1)}\right)_{-1}$ and $\left(\tilde{\varphi}^{(2)}\right)_{0}$, can be regarded as integrations functions, and that logarithmic terms do not appear if and only if (cf. condition (A.19)

$$
\left[\left(\frac{|\sigma|^{2}}{2^{3 / 2} x}-\frac{\kappa}{\sqrt{2} x}\right) \tilde{\varphi}^{(1)}-\frac{|\sigma|^{2}}{2 x} \tilde{\varphi}^{(2)}\right]_{0}=0 .
$$

Recall that $\cdot{ }_{n}$ denotes the $r^{-n}$-term $\left(x^{n}\right.$-term $)$ in the asymptotic expansion of the corresponding field. Using $\kappa=\mathcal{O}\left(r^{-3}\right)$ and $|\sigma|^{2}=\mathcal{O}\left(r^{-4}\right)$ we observe that (3.6) holds automatically. In particular we have $\varphi=\mathcal{O}(r)$. Furthermore, since

$$
\begin{aligned}
\left(\tilde{\varphi}^{(1)}\right)_{-1} & =-\sqrt{2}\left(\varphi^{(2)}\right)_{-1}=\sqrt{2} \varphi_{-1}, \\
\left(\tilde{\varphi}^{(2)}\right)_{0} & =\left(\varphi^{(1)}\right)_{0}+\left(\varphi^{(2)}\right)_{0}=\varphi_{0},
\end{aligned}
$$

the coefficients $\varphi_{-1}$ and $\varphi_{0}$ can be identified as the integration functions. As indicated above, though being left undetermined by the equation itself, they have global character.

In the following we shall set for convenience

$$
\sigma_{n}:=\left(|\sigma|^{2}\right)_{n} .
$$

Inserting the expansion $\varphi \sim \sum_{n=\hat{\ell}}^{\infty} \varphi_{n} r^{-n}$ into (3.5) and equating coefficients gives the expansion coefficients $\varphi_{n}$ by a hierarchy of equations,

$$
\begin{aligned}
\varphi_{1} & =\left(\frac{1}{2} \kappa_{3}-\frac{1}{4} \sigma_{4}\right) \varphi_{-1}, \\
\varphi_{2} & =\left(\frac{1}{6} \kappa_{4}-\frac{1}{12} \sigma_{5}\right) \varphi_{-1}-\frac{1}{12} \sigma_{4} \varphi_{0},
\end{aligned}
$$

while

$$
\begin{aligned}
\tau \equiv 2 \partial_{r} \log \varphi= & 2 r^{-1}-2 \varphi_{0}\left(\varphi_{-1}\right)^{-1} r^{-2} \\
& +\left[2\left(\varphi_{0}\right)^{2}\left(\varphi_{-1}\right)^{-2}+\sigma_{4}-2 \kappa_{3}\right] r^{-3}+\mathcal{O}\left(r^{-4}\right) .
\end{aligned}
$$

Consider the conformal factor relating the $r$-dependent Riemannian metric $\check{g}_{\Sigma_{r}}$ and $\gamma, \bar{g}_{A B} \mathrm{~d} x^{A} \mathrm{~d} x^{B}=\Omega^{2} \gamma_{A B} \mathrm{~d} x^{A} \mathrm{~d} x^{B}$. We find

$$
\begin{aligned}
\operatorname{det} \gamma= & \operatorname{det} s\left[r^{4}+h^{(1)} r^{3}+\left(h^{(2)}+\frac{1}{2}\left(h^{(1)}\right)^{2}-\frac{1}{2}\left|h^{(1)}\right|^{2}\right) r^{2}\right]+\mathcal{O}(r), \\
\Omega \equiv & \varphi\left(\frac{\operatorname{det} s}{\operatorname{det} \gamma}\right)^{1 / 4}=\varphi_{-1}-\frac{1}{4} \varphi_{-1}\left(2 \tau_{2}+h^{(1)}\right) r^{-1} \\
& +\frac{1}{4} \varphi_{-1}\left[2 \kappa_{3}+\frac{1}{4}\left(h^{(1)}\right)^{2}+\frac{1}{4}\left|h^{(1)}\right|^{2}-h^{(2)}+\frac{1}{2} \tau_{2} h^{(1)}\right] r^{-2}+\mathcal{O}\left(r^{-3}\right) .
\end{aligned}
$$

We conclude that

$$
\begin{aligned}
\bar{g}_{A B} & =\left(\varphi_{-1}\right)^{2}\left[s_{A B} r^{2}+\left(\breve{h}_{A B}^{(1)}-\tau_{2} s_{A B}\right) r\right. \\
& \left.+\breve{h}_{A B}^{(2)}-\left(\tau_{2}+\frac{1}{2} h^{(1)}\right) \breve{h}_{A B}^{(1)}+\left[\frac{1}{4}\left(\tau_{2}\right)^{2}+\kappa_{3}+\frac{1}{2} \sigma_{4}\right] s_{A B}\right]+\mathcal{O}\left(r^{-1}\right) .
\end{aligned}
$$

To sum it up, (2.15) and $\kappa=\mathcal{O}\left(r^{-3}\right)$ imply that no logarithmic terms appear in the conformal factor relating $\gamma_{A B}$ and $\bar{g}_{A B}$, the latter one thus being smoothly extendable at $\mathscr{I}^{+}$as a Riemannian metric on $S^{2}$ whenever a global solution of the Raychaudhuri equation exists on $\mathscr{N}$ with $\varphi_{-1} \neq 0$. 


\subsubsection{Expansion of $\nu^{0}$}

We consider the constraint equation (2.6) which determines $\nu^{0}$,

$$
\partial_{r} \nu^{0}+\nu^{0}\left(\frac{1}{2} \tau+\kappa\right)+\frac{1}{2}\left(\bar{W}^{0}+\overline{\hat{\Gamma}}^{0}\right)=0,
$$

where $\bar{W}^{0}=\mathcal{O}\left(r^{-1}\right)$ and, using (2.4),

$$
\begin{aligned}
\overline{\hat{\Gamma}}^{0} & =2 \nu^{0}\left(\hat{\nu}^{0} \partial_{r} \hat{\nu}_{0}-\hat{\kappa}\right)-\hat{\nu}^{0} \Omega^{-2} \gamma^{A B} \hat{\chi}_{A B} \\
& =\mathcal{O}\left(r^{-3}\right) \nu^{0}-2\left(\varphi_{-1}\right)^{-2} r^{-1}-2\left(\varphi_{-1}\right)^{-2} \tau_{2} r^{-2}+\mathcal{O}\left(r^{-3}\right) .
\end{aligned}
$$

Again, we express the ODE by $x \equiv 1 / r$. Its asymptotic form reads

$$
\begin{aligned}
{\left[x \partial_{x}\right.} & \left.-1-\frac{\tau_{2}}{2} x+\mathcal{O}\left(x^{2}\right)\right] \nu^{0} \\
& =\underbrace{\frac{1}{2}\left(\bar{W}^{0}\right)_{1}-\left(\varphi_{-1}\right)^{-2}}_{=:-\Phi^{-2}}+\left[\frac{1}{2}\left(\bar{W}^{0}\right)_{2}-\left(\varphi_{-1}\right)^{-2} \tau_{2}\right] x+\mathcal{O}\left(x^{2}\right) .
\end{aligned}
$$

We want to make sure that the asymptotic solution of $\nu^{0}$ can be written as power series. In the notation of Appendix $\mathrm{A}$ we have $\lambda=1$ and $\hat{\ell}=0$, and condition (A.10) which characterizes the absence of logarithmic terms reads

$$
\left(\bar{W}^{0}\right)_{2}=\left[\frac{1}{2}\left(\bar{W}^{0}\right)_{1}+\left(\varphi_{-1}\right)^{-2}\right] \tau_{2} .
$$

Assuming (3.17) we insert the expansion $\nu^{0} \sim \sum_{n=\hat{\ell}}^{\infty}\left(\nu^{0}\right)_{n} r^{-n}$ into (3.14) to obtain the expansion coefficients $\left(\nu^{0}\right)_{n}$ in terms of $\kappa, \bar{W}^{0}, \varphi$ and $\gamma$,

$$
\nu^{0}=\Phi^{-2}-D^{\left(\nu_{0}\right)} \Phi^{-4} r^{-1}+\mathcal{O}\left(r^{-2}\right),
$$

where $D^{\left(\nu_{0}\right)}$ denotes the globally defined integration function.

As mentioned above, for $\bar{g}_{\mu \nu}$ to have a smooth conformal completion at infinity the function $\left(\nu^{0}\right)_{0}$ needs to be of constant sign which is the case if and only if the gauge source function is chosen so that (compare Proposition 2.2)

$$
\left(\bar{W}^{0}\right)_{1} \neq 2\left(\varphi_{-1}\right)^{-2} \forall x^{A}
$$

For the inverse of $\nu^{0}$ we then find

$$
\nu_{0}=\Phi^{2}+D^{\left(\nu_{0}\right)} r^{-1}+\mathcal{O}\left(r^{-2}\right) .
$$

Note that in the special case where $\left(\bar{W}^{0}\right)_{1}=0$ we have $\Phi=\varphi_{-1}$ and the positivity of $\left(\nu^{0}\right)_{0}$ follows from that of $\varphi_{-1}$.

Since $\left(\bar{W}^{0}\right)_{1}$ can be prescribed arbitrarily and the value of $\varphi_{-1}$ does not depend on that choice, (3.19) is not a geometric restriction. Similarly, (3.17) can be fulfilled by an appropriate choice of $\left(\bar{W}^{0}\right)_{2}$. The gauge freedom associated with the choice of $\bar{W}^{0}$ can be used to control the behavior of $\nu^{0}$ and to get rid of the log terms in its asymptotic expansion (only the two leading order terms in the expansion of $\bar{W}^{0}$ are affected, compare the discussion in Section 3.3). 


\subsubsection{Expansion of $\xi_{A}$}

The connection coefficients $\xi_{A}=-2 \bar{\Gamma}_{r A}^{r}$ are determined by (2.7),

$$
\left(\partial_{r}+\tau\right) \xi_{A}-2 \check{\nabla}_{B} \sigma_{A}^{B}+\partial_{A} \tau+2 \partial_{A} \kappa=0 .
$$

To compute the covariant derivative of $\sigma_{A}{ }^{B}$ associated to $\bar{g}_{A B} \mathrm{~d} x^{A} \mathrm{~d} x^{B}$, we first determine the asymptotic form of the Christoffel symbols,

$\check{\Gamma}_{A B}^{C}=\frac{1}{2} \gamma^{C D}\left(2 \partial_{(A} \gamma_{B) D}-\partial_{D} \gamma_{A B}\right)+2 \delta_{(B}{ }^{C} \partial_{A)} \log \Omega-\gamma^{C D} \gamma_{A B} \partial_{D} \log \Omega=\mathcal{O}(1)$,

with

$$
\begin{aligned}
\left(\check{\Gamma}_{A B}^{C}\right)_{0}= & \stackrel{\circ}{\Gamma}_{A B}^{C}+2 \delta_{(B}{ }^{C} \stackrel{\circ}{\nabla}_{A)} \log \varphi_{-1}-s_{A B} \stackrel{\circ}{\nabla}^{C} \log \varphi_{-1}, \\
\left(\check{\Gamma}_{A B}^{C}\right)_{1}= & \stackrel{\circ}{\nabla}_{(A} \breve{h}_{B)}^{(1) C}-\frac{1}{2} \stackrel{\circ}{\nabla}^{C} \breve{h}_{A B}^{(1)}-\delta_{(A}{ }^{C} \stackrel{\circ}{\nabla}_{B)} \tau_{2}+\frac{1}{2} s_{A B} \stackrel{\circ}{\nabla}^{C} \tau_{2} \\
& -\left(s^{C D} h_{A B}^{(1)}-h^{(1) C D} s_{A B}\right) \stackrel{\circ}{\nabla}_{D} \log \varphi_{-1} .
\end{aligned}
$$

Invoking (3.3) that yields

$$
\check{\nabla}_{B} \sigma_{A}^{B}=\Xi_{A}^{(2)} r^{-2}+\Xi_{A}^{(3)} r^{-3}+\mathcal{O}\left(r^{-4}\right),
$$

where

$$
\begin{aligned}
& \Xi_{A}^{(2)}=-\frac{1}{2} \stackrel{\circ}{\nabla}_{B} \breve{h}_{A}^{(1) B}-\breve{h}_{A}^{(1) B} \stackrel{\circ}{\nabla}_{B} \log \varphi_{-1}=\stackrel{\circ}{\nabla}_{B}\left(\sigma_{A}^{B}\right)_{2}+2\left(\sigma_{A}^{B}\right)_{2} \stackrel{\circ}{\nabla}_{B} \log \varphi_{-1} \\
& \Xi_{A}^{(3)}=\stackrel{\circ}{\nabla}_{B}\left(\sigma_{A}{ }^{B}\right)_{3}+2\left(\sigma_{A}{ }^{B}\right)_{3} \stackrel{\circ}{\nabla}_{B} \log \varphi_{-1}-\left(\sigma_{A}^{B}\right)_{2} \stackrel{\circ}{\nabla}_{B} \tau_{2}+\frac{1}{2} \stackrel{\circ}{\nabla}_{A} \sigma_{4} .
\end{aligned}
$$

Substituting now the coefficients by their asymptotic expansions we observe that (3.21) has the asymptotic structure,

$$
\begin{aligned}
& \left(\partial_{r}+2 r^{-1}+\tau_{2} r^{-2}+\mathcal{O}\left(r^{-3}\right)\right) \xi_{A} \\
& \quad=\left(2 \Xi_{A}^{(2)}-\partial_{A} \tau_{2}\right) r^{-2}+\left[2 \Xi_{A}^{(3)}-\partial_{A}\left(\tau_{3}+2 \kappa_{3}\right)\right] r^{-3}+\mathcal{O}\left(r^{-4}\right) .
\end{aligned}
$$

Nicely enough, the ODEs for $\xi_{A}, A=2,3$, are decoupled. For comparison with the formulae in Appendix $₫$ we rewrite them in terms of $x \equiv 1 / r$,

$$
\begin{aligned}
\left(x \partial_{x}\right. & \left.-2-\tau_{2} x+\mathcal{O}\left(x^{2}\right)\right) \xi_{A} \\
\quad & =\left(\partial_{A} \tau_{2}-2 \Xi_{A}^{(2)}\right) x-\left[2 \Xi_{A}^{(3)}-\partial_{A}\left(\tau_{3}+2 \kappa_{3}\right)\right] x^{2}+\mathcal{O}\left(x^{3}\right) .
\end{aligned}
$$

Appendix $₫$ tells us (with $\lambda=2$ and $\hat{\ell}=1$ ) that there are no logarithmic terms in the expansion of $\xi_{A}$ if and only if A.10 holds,

$$
\tau_{2}\left(2 \Xi_{A}^{(2)}-\partial_{A} \tau_{2}\right)=2 \Xi_{A}^{(3)}-\stackrel{\circ}{\nabla}_{A}\left(\tau_{3}+2 \kappa_{3}\right) .
$$

The asymptotic expansion (3.10) of $\tau$ implies

$$
\tau_{3}=\frac{1}{2}\left(\tau_{2}\right)^{2}+\sigma_{4}-2 \kappa_{3},
$$

such that (3.27) can be written as

$$
2 \tau_{2} \Xi_{A}^{(2)}=2 \Xi_{A}^{(3)}-\stackrel{\circ}{\nabla}_{A} \sigma_{4}
$$


Note that $\kappa_{3}$, on which we have not imposed conditions yet, drops out, so there is no gauge freedom left which could be appropriately adjusted to fulfill this equation. The impact of (3.29) will be analyzed in Section 3.2 where it becomes manifest that it does impose geometric restrictions on the initial data. We refer to (3.29) as no-logs-condition.

Whenever the no-logs-condition holds, which we assume henceforth, the covector field $\xi_{A}$ can be expanded as a power series,

$$
\xi_{A}=\left(2 \Xi_{A}^{(2)}-\stackrel{\circ}{\nabla}_{A} \tau_{2}\right) r^{-1}+C_{A}^{\left(\xi_{B}\right)} r^{-2}+\mathcal{O}\left(r^{-3}\right) .
$$

The coefficients $C_{A}^{\left(\xi_{B}\right)}=C_{A}^{\left(\xi_{B}\right)}\left(x^{C}\right), A=2,3$, represent globally defined integration functions.

\subsubsection{Expansion of $\nu_{A}$}

We analyze (2.8) to compute the asymptotic behavior of $\nu_{A}$,

$$
\left[2 \partial_{r}-\nu_{0}\left(\bar{W}^{0}+\overline{\hat{\Gamma}}^{0}\right)\right] \nu_{A}-4 \nu_{B} \chi_{A}^{B}+\nu_{0}\left[\xi_{A}-\bar{g}_{A B}\left(\bar{W}^{B}+\overline{\hat{\Gamma}}^{B}\right)+\gamma_{A B} \gamma^{C D} \check{\Gamma}_{C D}^{B}\right]=0
$$

Here

$$
\begin{aligned}
\nu_{0} \bar{g}_{A B} \overline{\hat{\Gamma}}^{B} & =2 \bar{g}_{A B} \overline{\hat{\Gamma}}_{01}^{B}-2 \bar{g}_{A B} \nu^{C} \hat{\chi}_{C}{ }^{B}+\nu_{0} \gamma_{A B} \gamma^{C D}\left(\hat{\tilde{\Gamma}}_{C D}^{B}-\overline{\hat{g}}^{1 B} \hat{\chi}_{C D}\right) \\
& =-\hat{\tau} \nu_{A}+v_{A}{ }^{B} \nu_{B}+\nu_{0} \gamma_{A B} \gamma^{C D}{ }^{\circ}{ }_{C D}^{B}+\mathcal{O}\left(r^{-2}\right)
\end{aligned}
$$

with $v_{C}{ }^{B}=\mathcal{O}\left(r^{-2}\right) \in \operatorname{Mat}(2,2)$, as follows from 2.4 . Recall that $\bar{W}^{0}=$ $\mathcal{O}\left(r^{-1}\right)$ and $\bar{W}^{A}=\mathcal{O}\left(r^{-1}\right)$, or, by (3.13), $\bar{W}_{A}:=\bar{g}_{A B} \bar{W}^{B}=\mathcal{O}(r)$. We determine the asymptotic expansions of the coefficients involved,

$$
\begin{aligned}
\nu_{B} \chi_{A}{ }^{B} & \equiv \frac{1}{2} \nu_{B} \bar{g}^{B C} \partial_{r} \bar{g}_{A C}=\nu_{A} \partial_{r} \log \Omega+\frac{1}{2} \nu_{B} \gamma^{B C} \partial_{r} \gamma_{A C} \\
& =\nu_{A} r^{-1}+\frac{1}{2}\left(\tau_{2} \nu_{A}-\breve{h}_{A}^{(1) B} \nu_{B}\right) r^{-2}+\mathcal{O}\left(r^{-3}\right) .
\end{aligned}
$$

For the source term involving Christoffel symbols of the metrics $s_{A B}$ and $\bar{g}_{A B}=$ $\Omega^{2} \gamma_{A B}$ we obtain,

$$
\gamma_{A B} \gamma^{C D}\left(\stackrel{\circ}{\Gamma}_{C D}^{B}-\check{\Gamma}_{C D}^{B}\right)=-\stackrel{\circ}{\nabla}_{B} \breve{h}_{A}^{(1) B} r^{-1}+\mathcal{O}\left(r^{-2}\right)
$$

Combining this with what we found for $\nu_{0}, \overline{\hat{\Gamma}}^{0}$ and $\xi_{A}$ (assuming that the nologs-condition holds) the ODE for $\nu_{A}$ takes the asymptotic form

$$
\begin{array}{r}
\partial_{r} \nu_{A}+w_{A}{ }^{B} \nu_{B}=\frac{\Phi^{2}}{2}\left(\bar{W}_{A}\right)_{-1} r+\frac{1}{2}\left[\Phi^{2}\left(\bar{W}_{A}\right)_{0}+D^{\left(\nu_{0}\right)}\left(\bar{W}_{A}\right)_{-1}\right]+\frac{1}{2}\left[\left(\nu_{0}\right)_{2}\left(\bar{W}_{A}\right)_{-1}\right. \\
\left.+D^{\left(\nu_{0}\right)}\left(\bar{W}_{A}\right)_{0}+\Phi^{2}\left(\stackrel{\circ}{\nabla}_{A} \tau_{2}+2 \breve{h}_{A}^{(1) B} \stackrel{\circ}{B}_{B} \log \varphi_{-1}+\left(\bar{W}_{A}\right)_{1}\right)\right] r^{-1}+\mathcal{O}\left(r^{-2}\right)
\end{array}
$$

where

$$
\begin{aligned}
w_{A}^{B} & =\left[\frac{\hat{\tau}}{2}-\frac{1}{2} \nu_{0}\left(\bar{W}^{0}+\overline{\hat{\Gamma}}^{0}\right)\right] \delta_{A}^{B}-2 \chi_{A}{ }^{B} \\
& =\left[\left[\Phi^{-2} D^{\left(\nu_{0}\right)}-\frac{1}{2} \tau_{2}\right] \delta_{A}^{B}-2\left(\sigma_{A}^{B}\right)_{2}\right] r^{-2}+\mathcal{O}\left(r^{-3}\right) \in \operatorname{Mat}(2,2) .
\end{aligned}
$$


Note that this first-order system is not decoupled. In terms of $x \equiv 1 / r$ the equation becomes $\left(\operatorname{set} \tilde{w}_{A}^{B}\left(x, x^{A}\right)=-x^{-1} w_{A}{ }^{B}\left(x^{-1}, x^{A}\right)=\mathcal{O}(x)\right)$

$$
\begin{aligned}
& x \partial_{x} \nu_{A}+\tilde{w}_{A}{ }^{B} \nu_{B}=-\frac{1}{2} \Phi^{2}\left(\bar{W}_{A}\right)_{-1} x^{-2}-\frac{1}{2}\left[\Phi^{2}\left(\bar{W}_{A}\right)_{0}+D^{\left(\nu_{0}\right)}\left(\bar{W}_{A}\right)_{-1}\right] x^{-1} \\
& -\frac{1}{2}\left[\left(\nu_{0}\right)_{2}\left(\bar{W}_{A}\right)_{-1}+D^{\left(\nu_{0}\right)}\left(\bar{W}_{A}\right)_{0}+\Phi^{2}\left(\stackrel{\circ}{\nabla}_{A} \tau_{2}+2 \breve{h}_{A}^{(1) B} \stackrel{\circ}{\nabla}_{B} \log \varphi_{-1}+\left(\bar{W}_{A}\right)_{1}\right)\right]+\mathcal{O}(x) .
\end{aligned}
$$

Again, we consult Appendix $\AA$ inspection of A.19) tells us (with $\lambda_{1}=\lambda_{2}=0$, $\hat{\ell}=-2$ ) that no logarithmic terms appear whenever the source satisfies,

$$
\begin{aligned}
\left(\bar{W}_{A}\right)_{1}= & 4\left(\sigma_{A}^{B}\right)_{2} \stackrel{\circ}{B}_{B} \log \varphi_{-1}-\stackrel{\circ}{\nabla}_{A} \tau_{2}-\Phi^{-2}\left(\nu_{0}\right)_{2}\left(\bar{W}_{A}\right)_{-1} \\
& -\Phi^{-2} D^{\left(\nu_{0}\right)}\left(\bar{W}_{A}\right)_{0}-\frac{1}{2}\left[\left(\tilde{w}_{A}^{B}\right)_{1}\left(\tilde{w}_{B}^{C}\right)_{1}+\left(\tilde{w}_{A}^{C}\right)_{2}\right]\left(\bar{W}_{C}\right)_{-1} \\
& -\left(\tilde{w}_{A}^{B}\right)_{1}\left[\left(\bar{W}_{B}\right)_{0}+\Phi^{-2} D^{\left(\nu_{0}\right)}\left(\bar{W}_{B}\right)_{-1}\right] .
\end{aligned}
$$

We express (3.34) in terms of $\bar{W}^{A}=\bar{g}^{A B} \bar{W}_{B}$. It can be solved for $\left(\bar{W}^{A}\right)_{1}$, and thus provides a condition on $\left(\bar{W}^{A}\right)_{1}$ once $\left(\bar{W}^{A}\right)_{-1}$ and $\left(\bar{W}^{A}\right)_{0}$ have been chosen.

Assuming that the gauge functions $\bar{W}^{A}$ have an asymptotic behavior which fulfills (3.34), any solution of (3.31) has the asymptotic form $\nu_{A} \sim \sum_{n=\hat{\ell}}^{\infty}\left(\nu_{A}\right)_{n} r^{-n}$. For suitable, globally defined integration functions $D_{A}^{\left(\nu_{A}\right)}$ we find,

$$
\begin{aligned}
\nu_{A}= & \frac{\Phi^{2}}{4}\left(\bar{W}_{A}\right)_{-1} r^{2}+\left[\frac{\Phi^{2}}{2}\left[\left(\bar{W}_{A}\right)_{0}+\left(\sigma_{A}^{B}\right)_{2}\left(\bar{W}_{B}\right)_{-1}+\frac{\tau_{2}}{4}\left(\bar{W}_{A}\right)_{-1}\right]\right. \\
& \left.+\frac{1}{4} D^{\left(\nu_{0}\right)}\left(\bar{W}_{A}\right)_{-1}\right] r+D_{A}^{\left(\nu_{A}\right)}+\mathcal{O}\left(r^{-1}\right) .
\end{aligned}
$$

\subsubsection{Expansion of $\zeta$}

We consider the ODE (2.9) which determines the auxiliary function $\zeta$,

$$
\left(\partial_{r}+\tau+\kappa\right) \zeta+\check{R}-\frac{1}{2} \xi_{A} \xi^{A}+\check{\nabla}_{A} \xi^{A}=0 .
$$

It remains to compute the source terms. From (3.13) and (3.30) we find

$$
\begin{aligned}
\frac{1}{2} \bar{g}^{A B} \xi_{A} \xi_{B} & =\mathcal{O}\left(r^{-4}\right) \\
\bar{g}^{A B} \check{\nabla}_{A} \xi_{B} & =\left(\varphi_{-1}\right)^{-2} \stackrel{\circ}{\nabla}^{A}\left(\xi_{A}\right)_{1} r^{-3}+\mathcal{O}\left(r^{-4}\right),
\end{aligned}
$$

with

$$
\left(\xi_{A}\right)_{1}=2\left(\check{\nabla}_{B} \sigma_{A}^{B}\right)_{2}-\stackrel{\circ}{\nabla}_{A} \tau_{2} .
$$

LEMMA 3.1 The curvature scalar $\check{R}$ satisfies

$$
\check{R}=\check{R}_{2} r^{-2}+\left[\tau_{2} \check{R}_{2}-\left(\varphi_{-1}\right)^{-2} \stackrel{\circ}{\nabla}^{A}\left(\xi_{A}\right)_{1}\right] r^{-3}+\mathcal{O}\left(r^{-4}\right),
$$

with $\check{R}_{2}=2\left(\varphi_{-1}\right)^{-2}\left(1-\Delta_{s} \log \varphi_{-1}\right)$. 
Since the computation of the curvature scalar is elementary though somewhat lengthy we leave the proof of Lemma 3.1 to the reader.

Putting everything together, we observe that the $\zeta$-constraint is of the form

$$
\left[\partial_{r}+\frac{2}{r}+\tau_{2} r^{-2}+\mathcal{O}\left(r^{-3}\right)\right] \zeta=-\check{R}_{2} r^{-2}-\tau_{2} \check{R}_{2} r^{-3}+\mathcal{O}\left(r^{-4}\right) .
$$

We express the ODE in terms of $x \equiv 1 / r$,

$$
\left[x \partial_{x}-2-\tau_{2} x+\mathcal{O}\left(x^{2}\right)\right] \zeta=\check{R}_{2} x+\tau_{2} \check{R}_{2} x^{2}+\mathcal{O}\left(x^{3}\right) .
$$

Comparison with Appendix $\mathrm{A}$ tells us (with $\lambda=2$ and $\hat{\ell}=1$ ) that, due to the specific form of the source, condition (A.10), which excludes the appearance of logarithmic terms, is automatically satisfied, and the asymptotic expansion of $\zeta$ reads

$$
\zeta=-\check{R}_{2} r^{-1}+C^{(\zeta)} r^{-2}+\mathcal{O}\left(r^{-3}\right),
$$

where $C^{(\zeta)}=C^{(\zeta)}\left(x^{A}\right)$ can be identified with the integration function. It thus follows that $\zeta=\mathcal{O}\left(r^{-1}\right)$, supposing that $\kappa=\mathcal{O}\left(r^{-3}\right)$, that $\gamma$ has the asymptotic form (2.15) with $\varphi_{-1} \neq 0$, and that the no-logs-condition holds.

\subsubsection{Expansion of $\bar{g}^{r r}$}

Let us compute $\bar{g}^{r r}$ which satisfies (2.10),

$$
\left(\partial_{r}+\frac{1}{2} \tau+\kappa\right) \bar{g}^{r r}=\frac{1}{2} \zeta-\bar{W}^{r}-\overline{\hat{\Gamma}}^{r} .
$$

Recall that now where we have established (3.40), it follows from the a priori restrictions that $\bar{W}^{r}=\mathcal{O}(r)$. We further have,

$$
\begin{aligned}
\overline{\hat{\Gamma}}^{r} & =2 \nu^{0} \hat{\Gamma}_{0 r}^{r}+\bar{g}^{r r} \hat{\kappa}-\bar{g}^{r A} \hat{\xi}_{A}+\bar{g}^{A B} \hat{\Gamma}_{A B}^{r} \\
& =\hat{\kappa} \bar{g}^{r r}-2\left(\varphi_{-1}\right)^{-2} r^{-1}-2\left(\varphi_{-1}\right)^{-2} \tau_{2} r^{-2}+\mathcal{O}\left(r^{-3}\right),
\end{aligned}
$$

so the ODE for $\bar{g}^{r r}$ is of the form

$$
\left(\partial_{r}+r^{-1}+\mathcal{O}\left(r^{-2}\right)\right) \bar{g}^{r r}=\mathcal{O}(r),
$$

or, expressed in terms of the $x \equiv 1 / r$-coordinate,

$$
\left(x \partial_{x}-1+f^{*}\right) \bar{g}^{r r}=f^{* *},
$$

where

$$
\begin{aligned}
f^{*}= & -\frac{1}{2} \tau_{2} x-\left(\frac{1}{2} \tau_{3}+\kappa_{3}+\hat{\kappa}_{3}\right) x^{2}-\left(\frac{1}{2} \tau_{4}+\kappa_{4}+\hat{\kappa}_{4}\right) x^{3}+\mathcal{O}\left(x^{4}\right) \\
f^{* *}= & \left(\bar{W}^{r}\right)_{-1} x^{-2}+\left(\bar{W}^{r}\right)_{0} x^{-1}+\left(\bar{W}^{r}\right)_{1}+\frac{1}{2} \check{R}_{2}-2\left(\varphi_{-1}\right)^{-2} \\
& +\left[\left(\bar{W}^{r}\right)_{2}-\frac{1}{2} \zeta_{2}-2\left(\varphi_{-1}\right)^{-2} \tau_{2}\right] x+\mathcal{O}\left(x^{2}\right)
\end{aligned}
$$

We analyze the occurrence of logarithmic terms in the asymptotic solution of (3.42). In the notation of Appendix $\AA$ we have $\lambda=1$ and $\hat{\ell}=-2$. The 
considerations made there show that the asymptotic expansion of $\bar{g}^{r r}$ is $\mathcal{O}\left(r^{2}\right)$ if and only if the following condition is fulfilled (cf. (A.10) ),

$$
f_{1}^{*}\left(\bar{g}^{r r}\right)_{0}+f_{2}^{*}\left(\bar{g}^{r r}\right)_{-1}+f_{3}^{*}\left(\bar{g}^{r r}\right)_{-2}=f_{1}^{* *} .
$$

The expansion coefficients $\left(\bar{g}^{11}\right)_{i}$ can be derived from (A.9),

$$
\begin{aligned}
\left(\bar{g}^{r r}\right)_{-2} & =-\frac{1}{3} f_{-2}^{* *}, \\
\left(\bar{g}^{r r}\right)_{-1} & =-\frac{1}{2} f_{-1}^{* *}-\frac{1}{6} f_{1}^{*} f_{-2}^{* *}, \\
\left(\bar{g}^{r r}\right)_{0} & =-f_{0}^{* *}-\frac{1}{3} f_{2}^{*} f_{-2}^{* *}-\frac{1}{2} f_{1}^{*} f_{-1}^{* *}-\frac{1}{6}\left(f_{1}^{*}\right)^{2} f_{-2}^{* *} .
\end{aligned}
$$

A straightforward calculation reveals that (3.43) is equivalent to

$$
\begin{aligned}
\left(\bar{W}^{r}\right)_{2}= & \frac{\zeta_{2}}{2}+\left(\varphi_{-1}\right)^{-2} \tau_{2}+\frac{\tau_{2}}{4} \check{R}_{2}+\frac{\tau_{2}}{2}\left(\bar{W}^{r}\right)_{1}+\left[\frac{\tau_{3}}{4}+\frac{\kappa_{3}+\hat{\kappa}_{3}}{2}-\frac{\left(\tau_{2}\right)^{2}}{8}\right]\left(\bar{W}^{r}\right)_{0} \\
& {\left[\frac{1}{48}\left(\tau_{2}\right)^{3}-\frac{\tau_{2}}{4}\left(\frac{\tau_{3}}{2}+\kappa_{3}+\hat{\kappa}_{3}\right)+\frac{\tau_{4}}{6}+\frac{1}{3}\left(\kappa_{4}+\hat{\kappa}_{4}\right)\right]\left(\bar{W}^{r}\right)_{-1} }
\end{aligned}
$$

By an appropriate choice of the gauge source function $\bar{W}^{r}$, or merely the expansion coefficient $\left(\bar{W}^{r}\right)_{2}$, one can always arrange that (3.44) holds and thereby get rid of all logarithmic terms in the expansion of $\bar{g}^{r r}$. In that case

$$
\begin{aligned}
\bar{g}^{r r} & =-\frac{1}{3}\left(\bar{W}^{r}\right)_{-1} r^{2}+\mathcal{O}(r) \\
\Longrightarrow \quad \bar{g}_{00} & =\Phi^{4}\left[\frac{1}{16}\left(\varphi_{-1}\right)^{2} s_{A B}\left(\bar{W}^{A}\right)_{1}\left(\bar{W}^{B}\right)_{1}+\frac{1}{3}\left(\bar{W}^{r}\right)_{-1}\right] r^{2}+\mathcal{O}(r) .
\end{aligned}
$$

The integration function is represented by $\left(\bar{g}_{00}\right)_{1}$, and its explicit form is not relevant here.

\subsection{The no-logs-condition}

The no-logs-condition (3.29)

$$
2 \tau_{2}\left[\check{\nabla}_{B} \sigma_{A}^{B}\right]_{2}=2\left[\check{\nabla}_{B} \sigma_{A}^{B}\right]_{3}-\stackrel{\circ}{\nabla}_{A} \sigma_{4},
$$

needs to be imposed to exclude logarithmic terms in the asymptotic expansion of $\xi_{A}$. Let us rewrite and simplify it. In (3.25) and (3.26) we have computed $\left[\tilde{\nabla}_{B} \sigma_{A}{ }^{B}\right]_{2}$ and $\left[\tilde{\nabla}_{B} \sigma_{A}{ }^{B}\right]_{3}$. Plugging this in, we observe that the $\stackrel{\circ}{\nabla}_{A} \sigma_{4}$-terms cancel out in (3.45), which becomes

$$
\stackrel{\circ}{\nabla}_{B}\left[\tau_{2}\left(\sigma_{A}^{B}\right)_{2}-\left(\sigma_{A}^{B}\right)_{3}\right]+2\left[\tau_{2}\left(\sigma_{A}^{B}\right)_{2}-\left(\sigma_{A}^{B}\right)_{3} \stackrel{\circ}{\nabla}_{B}\right] \stackrel{\circ}{\nabla}_{B} \log \varphi_{-1}=0 .
$$

This can be written as a divergence,

$$
\stackrel{\circ}{\nabla}_{B}\left[\left(\varphi_{-1}\right)^{2}\left(\tau_{2}\left(\sigma_{A}^{B}\right)_{2}-\left(\sigma_{A}^{B}\right)_{3}\right)\right]=0 .
$$

One can view this equation as a linear first-order PDE-system on $S^{2}$. Note that by definition of $\sigma_{A}{ }^{B}$ the expansion coefficients $\left(\sigma_{A}{ }^{B}\right)_{n}$ are $s$-traceless tensors on $S^{2}$, whence the term in squared brackets in (3.47) is traceless. It is known (cf. e.g. 9]) that for any smooth source $v_{A}$ a PDE-system of the form $\stackrel{\circ}{\nabla}_{B} \zeta_{A}{ }^{B}=v_{A}$, 
with $\zeta_{A}{ }^{B}$ a traceless tensor on the unit sphere $S^{2}$, admits precisely one smooth solution. In our case the source of the PDE vanishes, so we conclude that the no-logs-condition (3.45) is equivalent to (recall our assumption $\varphi_{-1} \neq 0$ )

$$
\left(\sigma_{A}^{B}\right)_{3}=\tau_{2}\left(\sigma_{A}^{B}\right)_{2} \Longleftrightarrow \breve{h}_{A B}^{(2)}=\frac{1}{2}\left(h^{(1)}+\tau_{2}\right) \breve{h}_{A B}^{(1)}
$$

Since $\xi_{A}=-2 \bar{\Gamma}_{r A}^{r}$, determined by (2.7), has a geometric meaning one should expect the no-logs-condition to be gauge-invariant. In [7] it is shown that this is indeed the case. Although the $\left(\kappa=0, \bar{W}^{0}=0\right)$-wave-map gauge invariably produces logarithmic terms except for the flat case 7], one can decide whether they can be transformed away or not by checking (3.48).

In the current scheme, where conformal data $\gamma$ are prescribed on $\mathscr{N}$, together with the gauge functions $\kappa$ and $W^{\lambda}$, this requires to determine $\tau_{2}$ by solving the Raychaudhuri equation, which makes this scheme not practical for the purpose. In particular, it is not a priori clear within this scheme whether any initial data satisfying this condition exist unless both $\left(\sigma^{A}{ }_{B}\right)_{2}$ and $\left(\sigma^{A}{ }_{B}\right)_{3}$ vanish $(\Longleftrightarrow$ $\breve{h}_{A B}^{(1)}=0=\breve{h}_{A B}^{(2)}$. On the other hand, in gauge schemes where $\check{g}$ is prescribed, (3.48) is a straightforward condition on its asymptotic behavior (cf. Section 4 where a related scheme is used).

In 7] we also provide a geometric interpretation of the no-logs-condition (3.48) via the conformal Weyl tensor. Moreover, (3.48) can be related to Bondi's "outgoing wave condition", cf. Section 4.5, Remark 4.1.

\subsection{Summary and discussion}

The subsequent theorem summarizes our analysis of the asymptotic behavior of solutions to Einstein's vacuum constraint equations ((i) and (ii) follow from [7]):

THEOREM 3.2 Consider the characteristic initial value problem for Einstein's vacuum field equations with smooth conformal data $\gamma=\gamma_{A B} \mathrm{~d} x^{A} \mathrm{~d} x^{B}$ and gauge functions $\kappa$ and $\bar{W}^{\lambda}$, all defined on a smooth characteristic surface $\mathscr{N}$ meeting $\mathscr{I}^{+}$transversally in a smooth spherical cross-section (and supplemented by certain data on the intersection manifold $S$ if $\mathscr{N}$ is one of two transversally intersecting null hypersurfaces). The following conditions are necessary-andsufficient for the trace of the metric $g=g_{\mu \nu} \mathrm{d} x^{\mu} \mathrm{d} x^{\nu}$ on $\mathscr{N}$, obtained as solution to the characteristic wave-map vacuum constraint equations (2.5)-(2.10), to admit a smooth conformal completion at infinity, and for the connection coefficients $\bar{\Gamma}_{r A}^{r}$ to be smooth at $\mathscr{I}^{+}$(in the sense of Definition 2.1), when imposing a generalized wave-map gauge condition $H^{\lambda}=0$ :

(i) There exists a one-parameter family $\varkappa=\varkappa(r)$ of Riemannian metrics on $S^{2}$ such that $\varkappa$ satisfies 2.15 ) and is conformal to $\gamma$ (in particular we may assume $\gamma$ itself to be of the form (2.15)).

(ii) The functions $\varphi, \nu^{0}, \varphi_{-1}$ and $\left(\nu_{0}\right)_{0}$ have no zeros on $\mathscr{N}$ and $S^{2}$, respectively (the non-vanishing of $\left(\nu^{0}\right)_{0}$ is equivalent to (3.19)).

(iii) The gauge source functions are chosen in such a way that $\kappa=\mathcal{O}\left(r^{-3}\right)$, $\bar{W}^{0}=\mathcal{O}\left(r^{-1}\right), \bar{W}^{A}=\mathcal{O}\left(r^{-1}\right), \bar{W}^{r}=\mathcal{O}(r)$, and such that the conditions 3.17, 3.34) and 3.44) hold. 
(iv) The no-logs-condition is satisfied, i.e.

$$
\left(\sigma_{A}{ }^{B}\right)_{3}=\tau_{2}\left(\sigma_{A}^{B}\right)_{2} \quad \Longleftrightarrow \quad \breve{h}_{A B}^{(2)}=\frac{1}{2}\left(h^{(1)}+\tau_{2}\right) \breve{h}_{A B}^{(1)} .
$$

REMARK 3.3 For further reference let us explicitly list the conditions (3.17), (3.34) and (3.44) in the case where the gauge source functions satisfy $\bar{W}^{0}=$ $\mathcal{O}\left(r^{-2}\right), \bar{W}^{A}=\mathcal{O}\left(r^{-3}\right), \bar{W}^{r}=\mathcal{O}\left(r^{-2}\right)$ :

$$
\begin{aligned}
\left(\bar{W}^{0}\right)_{2} & =\tau_{2}\left(\varphi_{-1}\right)^{-2}, \\
\left(\bar{W}^{A}\right)_{3} & =-\left(\varphi_{-1}\right)^{-2} \stackrel{\circ}{ }^{A} \tau_{2}+4\left(\varphi_{-1}\right)^{-3}\left(\sigma_{B}{ }^{A}\right)_{2} \stackrel{\circ}{ }^{B} \varphi_{-1}, \\
\left(\bar{W}^{r}\right)_{2} & =\frac{1}{2} \zeta_{2}+\tau_{2}\left[\left(\varphi_{-1}\right)^{-2}+\frac{1}{4} \check{R}_{2}+\frac{1}{2}\left(\bar{W}^{r}\right)_{1}\right] .
\end{aligned}
$$

If, in addition, the no-logs-condition (3.49) is fulfilled then the leading order terms of the non-vanishing metric components restricted to the cone read

$$
\begin{aligned}
\bar{g}_{00}= & -\left(1+\Delta_{s} \varphi_{-1}\right)+D^{\left(\bar{g}_{00}\right)} r^{-1}+\mathcal{O}\left(r^{-2}\right) \\
\nu_{0}= & \left(\varphi_{-1}\right)^{2}+D^{\left(\nu_{0}\right)} r^{-1}+\mathcal{O}\left(r^{-2}\right) \\
\nu_{A}= & D_{A}^{\left(\nu_{A}\right)}+\mathcal{O}\left(r^{-1}\right) \\
\bar{g}_{A B}= & \left(\varphi_{-1}\right)^{2} s_{A B} r^{2}+\left(\varphi_{-1}\right)^{2}\left[\breve{h}_{A B}^{(1)}-\tau_{2} s_{A B}\right] r \\
& -\frac{1}{2} \tau_{2} \breve{h}_{A B}^{(1)}+\left(\frac{1}{4}\left(\tau_{2}\right)^{2}+\kappa_{3}+\frac{1}{2} \sigma_{4}\right) s_{A B}+\mathcal{O}\left(r^{-1}\right) .
\end{aligned}
$$

The coefficients denoted by $D$ have a global character in that they are globally defined by the initial data and the gauge functions.

REMARK 3.4 For definiteness we have restricted attention to the setting where the initial data are provided by the conformal data $\gamma=\gamma_{A B} \mathrm{~d} x^{A} \mathrm{~d} x^{B}$ together with the gauge functions $\kappa$ and $W^{\lambda}$. Theorem 3.2, though, is quite independent of the particular setting that has been chosen. This is discussed in [7].

It is shown in Appendix $\AA$ that when analyzing the asymptotic behavior of a linear first-order Fuchsian ODE-system whose indicial matrix has only integer eigenvalues one has to distinguish two different cases. If the indicial matrix cannot be diagonalized, or if it can be but the condition A.20 of Appendix $\AA$ is violated, then the appearance of logarithmic terms always depends on the boundary conditions and thereby on the globally defined integration functions. If, however, A.20 is fulfilled their appearance depends exclusively on the asymptotic behavior of the coefficients in the corresponding ODE. In fact, all the Einstein wave-map gauge constraints are of the latter type 3

Due to this property and since many of the constraint equations have a source term which involves a gauge freedom, many, though not all, logarithmic terms arising can be eliminated for given data $\gamma$ by a carefully adjusted leadingorder-term-behavior of the gauge functions. These are precisely the conditions of Theorem 3.2 which involve a gauge source function $\kappa$ or $\bar{W}^{\lambda}$. Logarithmic terms which appear if these conditions are violated are pure gauge artifacts.

This does not apply to the $\xi_{A^{-}}$and the $\zeta$-equation. Recall that to solve the equation for $\xi_{A}$ both $\kappa$ and $\varphi$ need to be known. This requires a choice of the

\footnotetext{
${ }^{3}$ Since the integration functions of some constraint equations appear as coefficients in other ones, the no-logs-condition does depend on the integration functions $\varphi_{-1}$ and $\varphi_{0}$.
} 
$\kappa$-gauge. Since the choice of $\bar{W}^{0}$ does not affect the $\xi_{A}$-equation, there is no gauge-freedom left. If the no-logs-condition (3.49), which is gauge-invariant 7], does not hold, there is no possibility to get rid of the log terms that arise there, whatever $\kappa$ has been chosen to be. Similarly, there is no gauge-freedom left when the equation for $\zeta$ is integrated but, due to the special asymptotic structure of its source term, no new log terms arise in the expansion of $\zeta$.

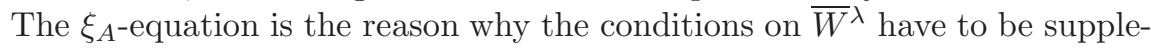
mented by the no-logs-condition (3.49) which involves two integration functions, $\varphi_{-1}$ and $\varphi_{0}$, globally determined by the initial data $\gamma$ and the gauge function $\kappa$. A decisive grievance is that, at least in our current setting, their dependence on $\gamma$ and $\kappa$ is very intricate. Thus the question arises for which class of initial data one finds a function $\kappa=\mathcal{O}\left(r^{-3}\right)$, such that the no-logs-condition holds, and accordingly what the geometric restrictions are for this to be possible. The only obvious fact is that (3.49) will be satisfied for sure whenever $\breve{h}_{A B}^{(1)}=\breve{h}_{A B}^{(2)}=0$.

For a "generic" choice of $\gamma$ and $\kappa$ one should expect that the expansion coefficient $\tau_{2}=-2 \varphi_{0}\left(\varphi_{-1}\right)^{-1}$ will not vanish. Equation (3.17) then shows that a $\bar{W}^{0}=0$-gauge (in particular the harmonic gauge) is not adequate for our purposes, since logarithmic terms can only be removed by a non-vanishing gauge source function $\bar{W}^{0} \neq 0$. This is illustrated best by the no-go result $[\underline{6}$, Theorem 3.1]. In order to fulfill (3.17) one needs a gauge choice for $\bar{W}^{0}$ which depends on the globally defined integration functions $\left(\varphi_{-1}, \varphi_{0}\right)$. This indicates the need of an initial data-dependent gauge choice to get rid of the logarithmic terms. (Note that the higher-order terms in the expansion of $\bar{W}^{0}$ do not affect the appearance of log terms.) We shall address this issue in the next sections.

\section{Metric gauge}

\subsection{Introduction}

Up to now we investigated the overall form of the asymptotic expansions of the trace of the metric on $\mathscr{N}$. It turned out that it is not possible to manifestly eliminate all logarithmic terms just by imposing restrictions on the asymptotic behavior of the gauge functions. Instead, an additional gauge-independent "nologs-condition" (3.49), which depends on some of the globally defined integration functions, needs to be fulfilled to ensure expansions at conformal infinity in terms of power series. Nonetheless, the current gauge scheme is unsatisfactory in that it seems hopeless to characterize those classes of initial data which satisfy (3.49).

In the following we will modify the scheme to allow for a better treatment of these problems at hand. We develop a gauge scheme which is adjusted to the initial data in such a way that we can solve at least some of the constraint equations analytically, so that the values of the troublesome functions $\varphi_{-1}$ and $\varphi_{0}$, which appeared hitherto as "integration functions" in the asymptotic solutions and which are related to the appearance of log terms via (3.49), can be computed explicitly. In view of the computation of all the fields appearing in the conformal field equations we will choose a gauge in which the components of the metric tensor take preferably simple values (at the expense of more complicated gauge functions $\kappa$ and $\left.W^{\lambda}\right)$. As before we shall adopt Rendall's point of view [10] and regard $[\gamma]$ (together with a choice of $\kappa$ ) as the free "physical" data. 


\subsection{Gauge scheme}

The trace of certain components of the vacuum Einstein equations on $\mathscr{N}$ together with the gauge condition $\bar{H}^{\lambda}=0$ can be used to determine the metric and some of its transverse derivatives on $\mathscr{N}$. Similarly, the remaining components of the Einstein equations on $\mathscr{N}$ as well as their transverse derivatives, $\overline{\partial_{0}^{n} R_{\mu \nu}}=0, n \in \mathbb{N}$, in combination with the gauge condition $H^{\lambda}=0$, provide a way to determine higher-order transverse derivatives of the metric on $\mathscr{N}$ when necessary. To compute all the fields which appear in the conformal field equations, such as e.g. the rescaled Weyl tensor, this is what needs to be done.

However, it is very convenient to exploit the gauge freedom contained in the vector field $W^{\lambda}$ to prescribe certain metric components and transverse derivatives thereof on $\mathscr{N}$, and treat the corresponding equations as equations for $W^{\lambda}$. Indeed, proceeding this way the computations below can be significantly simplified. It turns out that the most convenient way here is a mixture of schemes described in [6]: We still regard the conformal class $[\gamma]$ of $\check{g}_{\Sigma_{r}}$ as the free "nongauge"-initial data 4 Recall that up to this stage we have regarded $\kappa$ and $W^{\lambda}$ as gauge degrees of freedom. Now, instead of $\overline{W^{\lambda}}$ and $\overline{\partial_{0} W^{\lambda}}$ we shall show that it is possible to prescribe the functions

$$
\bar{g}_{0 \mu} \text { and } \overline{\partial_{0} g_{0 \mu}} \text { with } \nu_{0}=\bar{g}_{0 r} \neq 0 \text {. }
$$

Of course there are some restrictions coming from regularity when $\mathscr{N}$ is a lightcone, and from the requirement of the metric to be continuous at the intersection manifold when $\mathscr{N}$ is one of two intersecting null surfaces. Since we are mainly interested in prescribing these function for large values of $r$, this issue can be ignored for our purposes. Some comments are given in the course of this section.

Let us now explain how the above gauge scheme can be realized: First of all we solve the Raychaudhuri equation to compute $\bar{g}_{A B}$, where, as before, we assume that a global solution $\tau$ exists. Then we compute $\bar{W}^{\lambda}$ from the constraint equations (2.6), (2.8) and (2.10), a procedure which was introduced in [6] as an alternative scheme for integrating the null constraint equations. In order to make sure that we may prescribe $\overline{\partial_{0} g_{0 \mu}}$ rather than $\overline{\partial_{0} W^{\lambda}}$ we need to analyze the remaining Einstein equations on $\mathscr{N}$ (those components which have not already been used to derive the Einstein wave-map gauge constraints in [2]).

We impose a wave-map gauge condition $H^{\lambda}=0$ with arbitrarily prescribed gauge functions $\kappa$ and $W^{\lambda}$. From $\bar{H}^{\lambda}=0$ we obtain algebraic equations for $\overline{\partial_{0} g_{r r}}, \overline{\partial_{0} g_{r A}}$ and $\bar{g} A B \overline{\partial_{0} g_{A B}}$,

$$
\begin{aligned}
\overline{\partial_{0} g_{r r}}= & \tau \nu_{0}+\left(\nu_{0}\right)^{2}\left(\overline{\hat{\Gamma}}^{0}+\bar{W}^{0}\right) \\
\bar{g}^{A B} \overline{\partial_{0} g_{r B}}= & \check{\nabla}^{A} \nu_{0}-\partial_{r} \nu^{A}-\nu_{0} \bar{g}^{C D} \check{\Gamma}_{C D}^{A}-\bar{g}^{r A}\left(\overline{\partial_{0} g_{r r}}-\tau \nu_{0}\right) \\
& +\nu_{0}\left(\overline{\hat{\Gamma}^{A}}+\bar{W}^{A}\right) \\
\bar{g}^{A B} \overline{\partial_{0} g_{A B}}= & 2 \check{\nabla}_{A} \nu^{A}-\left(2 \partial_{r}+2 \tau-\nu^{0} \overline{\partial_{0} g_{r r}}\right)\left(\nu_{0} \bar{g}^{r r}\right)-2 \nu_{0}\left(\overline{\hat{\Gamma}}^{r}+\bar{W}^{r}\right)
\end{aligned}
$$

Recall that

$$
R_{\mu \nu}=\partial_{\alpha} \Gamma_{\mu \nu}^{\alpha}-\partial_{\mu} \Gamma_{\nu \alpha}^{\alpha}+\Gamma_{\mu \nu}^{\alpha} \Gamma_{\alpha \beta}^{\beta}-\Gamma_{\mu \beta}^{\alpha} \Gamma_{\nu \alpha}^{\beta}
$$

${ }^{4}$ We do not regard e.g. $\bar{g}_{\mu \nu}$ as the free data as in $[6]$ since we want to separate gauge degrees of freedom and physical initial data. 
Einstein equations $\breve{R}_{A B}=0$ : Using the formulae in [2, Appendix A] (which we shall make extensively use of for all the subsequent computations) we find

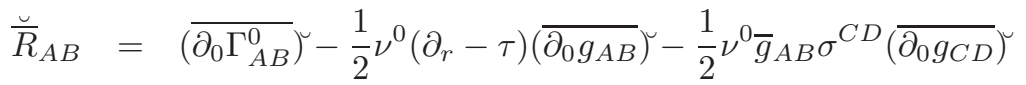

$$
\begin{aligned}
& +2 \nu^{0} \sigma_{(B}^{C}\left(\overline{\partial_{|0|} g_{A) C}}\right)-\left(\nu^{0}\right)^{2} \overline{\partial_{0} g_{0 r}} \sigma_{A B}+\text { known quantities } \\
& =-\nu^{0} \partial_{r}\left(\overline{\partial_{0} g_{A B}}\right)^{\top}+\frac{1}{2}\left(2 \tau \nu^{0}+\bar{W}^{0}+\overline{\hat{\Gamma}}^{0}\right)\left(\overline{\partial_{0} g_{A B}}\right)^{\top}+2 \nu^{0} \sigma_{(A}^{C}\left(\overline{\left.\partial_{|0|} g_{B}\right)}\right)^{\nu} \\
& + \text { known quantities , }
\end{aligned}
$$

where we used that

$$
\begin{aligned}
\overline{\partial_{0} \Gamma_{A B}^{0}}= & \frac{1}{2}\left(\nu^{0}\right)^{2} \overline{\partial_{0} g_{r r}} \overline{\partial_{0} g_{A B}}+\left(\nu^{0}\right)^{2} \overline{\partial_{0} g_{0 r}} \chi_{A B}-\frac{1}{2} \nu^{0} \partial_{r} \overline{\partial_{0} g_{A B}} \\
& + \text { known quantities . }
\end{aligned}
$$

Note that $\overline{\partial_{0} g_{0 r}}$, which appears in an intermediate step, cancels out at the end. We observe that $\breve{R}_{A B}=0$ provides a coupled linear ODE-system for $\left(\overline{\partial_{0} g_{A B}}\right)$.

The relevant boundary condition if $\mathscr{N}$ is a light-cone is [2, Section 4.5] $\lim _{r \rightarrow 0}\left(\overline{\partial_{0} g_{A B}}\right)^{u}=0$, in the case of two transversally intersecting characteristic surfaces the boundary condition is determined by the shear of $\mathscr{N}_{2}$.

Einstein equation $\bar{R}_{0 r}=0$ : We have

$$
\begin{aligned}
\bar{R}_{0 r} & =-\overline{\partial_{0} \Gamma_{r r}^{r}}-\overline{\partial_{0} \Gamma_{r A}^{A}}+\text { known quantities } \\
& =\frac{1}{2} \nu^{0} \overline{\partial_{00}^{2} g_{r r}}-\nu^{0}\left(\partial_{r}-\kappa\right) \overline{\partial_{0} g_{0 r}}+\text { known quantities },
\end{aligned}
$$

when taking into account that

$$
\begin{aligned}
\overline{\partial_{0} \Gamma_{r r}^{r}} & =\nu^{0}\left(\partial_{r} \overline{\partial_{0} g_{0 r}}-\frac{1}{2} \overline{\partial_{00}^{2} g_{r r}}\right)-\left(\nu^{0}\right)^{2}\left(\partial_{r} \nu_{0}-\frac{1}{2} \overline{\partial_{0} g_{r r}}\right) \overline{\partial_{0} g_{0 r}}+\text { known quantities }, \\
\overline{\partial_{0} \Gamma_{r B}^{A}} & =\text { known quantities. }
\end{aligned}
$$

Moreover, employing

$$
\begin{aligned}
& \overline{\partial_{0} \Gamma_{0 r}^{0}}=\frac{1}{2} \nu^{0} \overline{\partial_{00}^{2} g_{r r}}-\frac{1}{2}\left(\nu^{0}\right)^{2} \overline{\partial_{0} g_{r r}} \overline{\partial_{0} g_{0 r}}+\text { known quantities } \\
& \overline{\partial_{0} \Gamma_{i r}^{0}}=\text { known quantities }
\end{aligned}
$$

we find that

$$
\begin{aligned}
\overline{\partial_{0} H^{0}}= & \overline{\partial_{0} g^{\mu \nu}}\left(\bar{\Gamma}_{\mu \nu}^{0}-\overline{\hat{\Gamma}}_{\mu \nu}^{0}\right)+\bar{g}^{\mu \nu}\left(\overline{\partial_{0} \Gamma_{\mu \nu}^{0}}-\overline{\partial_{0} \hat{\Gamma}_{\mu \nu}^{0}}\right)-\overline{\partial_{0} W^{0}} \\
= & 2 \nu^{0} \overline{\partial_{0} \Gamma_{0 r}^{0}}+\bar{g}^{r r} \overline{\partial_{0} \Gamma_{r r}^{0}}+2 \bar{g}^{r A} \overline{\partial_{0} \Gamma_{r A}^{0}}+\bar{g}^{A B} \overline{\partial_{0} \Gamma_{A B}^{0}}-2\left(\nu^{0}\right)^{3} \overline{\partial_{0} g_{r r}} \overline{\partial_{0} g_{0 r}} \\
& -2\left(\nu^{0}\right)^{2} \overline{\partial_{0} g_{0 r}} \hat{\Gamma}_{0 r}^{0}-\overline{\partial_{0} W^{0}}+\text { known quantities } \\
= & \left(\nu^{0}\right)^{2} \overline{\partial_{00}^{2} g_{r r}}-\left[2\left(\nu^{0}\right)^{2}\left(\tau+\hat{\Gamma}_{0 r}^{0}\right)+3 \nu^{0}\left(\overline{W^{0}}+\overline{\hat{\Gamma}}^{0}\right)\right] \overline{\partial_{0} g_{0 r}}-\overline{\partial_{0} W^{0}} \\
& + \text { known quantities . }
\end{aligned}
$$

Consequently, the gauge condition $\overline{\partial_{0} H^{0}}=0$ can be used to rewrite the Einstein equation $\bar{R}_{0 r}=0$ as a linear ODE for $\overline{\partial_{0} g_{0 r}}$, or, depending on the setting, as an algebraic equation for the gauge source function $\overline{\partial_{0} W^{0}}$,

$$
\left[\partial_{r}-\tau-\kappa-\hat{\Gamma}_{0 r}^{0}-\frac{3}{2} \nu_{0}\left(\bar{W}^{0}+\overline{\hat{\Gamma}}^{0}\right)\right] \overline{\partial_{0} g_{0 r}}=\frac{1}{2}\left(\nu_{0}\right)^{2} \overline{\partial_{0} W^{0}}+\text { known quantities . }
$$


The boundary condition if $\mathscr{N}$ is a light-cone is [2, Section 4.5] $\lim _{r \rightarrow 0} \overline{\partial_{0} g_{0 r}}=0$; in the case of two characteristic surfaces intersecting transversally it is determined by $\left.\lim _{u \rightarrow 0} \partial_{u} g_{u r}\right|_{\mathscr{N}_{2}}$.

Once $\overline{\partial_{0} g_{0 r}}$ (or $\overline{\partial_{0} W^{0}}$ ) has been determined, we obtain $\overline{\partial_{00}^{2} g_{r r}}$ algebraically from the gauge condition $\overline{\partial_{0} H^{0}}=0$.

Einstein equations $\bar{R}_{0 A}=0$ : We find

$$
\begin{aligned}
\bar{R}_{0 A} & =-\overline{\partial_{0} \Gamma_{r A}^{r}}-\overline{\partial_{0} \Gamma_{A B}^{B}}+\nu^{0} \chi_{A} B \overline{\partial_{0} g_{0 B}}+\text { known quantities } \\
& =\frac{1}{2} \nu^{0} \overline{\partial_{00}^{2} g_{r A}}-\frac{1}{2} \nu^{0} \partial_{r} \overline{\partial_{0} g_{0 A}}+\nu^{0} \chi_{A}{ }^{B} \overline{\partial_{0} g_{0 B}}+\text { known quantities },
\end{aligned}
$$

where we used that

$$
\begin{aligned}
\overline{\partial_{0} \Gamma_{r A}^{r}} & =-\nu^{0} \chi_{A}{ }^{B} \overline{\partial_{0} g_{0 B}}+\frac{1}{2} \nu^{0}\left(\partial_{r} \overline{\partial_{0} g_{0 A}}-\overline{\partial_{00}^{2} g_{r A}}\right)+\text { known quantities } \\
\overline{\partial_{0} \Gamma_{A B}^{C}} & =\nu^{0} \chi_{A B} \bar{g}^{C D} \overline{\partial_{0} g_{0 D}}+\text { known quantities }
\end{aligned}
$$

From

$\overline{\partial_{0} \Gamma_{0 r}^{A}}=-\frac{1}{2} \nu^{0} \bar{g}^{A B} \overline{\partial_{0} g_{r r}} \overline{\partial_{0} g_{0 B}}+\frac{1}{2} \bar{g}^{A B}\left(\overline{\partial_{00}^{2} g_{r B}}+\partial_{r} \overline{\partial_{0} g_{0 B}}\right)+$ known quantities, $\overline{\partial_{0} \Gamma_{r r}^{A}}=$ known quantities ,

we obtain for the angle-components of the $u$-differentiated wave-gauge vector,

$$
\begin{aligned}
& \overline{\partial_{0} H^{A}}=\overline{\partial_{0} g^{\mu \nu}}\left(\bar{\Gamma}_{\mu \nu}^{A}-\hat{\Gamma}_{\mu \nu}^{A}\right)+\bar{g}^{\mu \nu}\left(\overline{\partial_{0} \Gamma_{\mu \nu}^{A}}-\overline{\partial_{0} \hat{\Gamma}_{\mu \nu}^{A}}\right)-\overline{\partial_{0} W^{A}} \\
& =-\left(\nu^{0}\right)^{2} \overline{\partial_{0} g_{r r}} \bar{g}^{A B} \overline{\partial_{0} g_{0 B}}-2 \nu^{0} \bar{g}^{B C} \overline{\partial_{0} g_{0 C}}\left(\chi_{B}{ }^{A}-\hat{\chi}_{B}{ }^{A}\right)+2 \nu^{0} \overline{\partial_{0} \Gamma_{0 r}^{A}} \\
& +\bar{g}^{i j} \overline{\partial_{0} \Gamma_{i j}^{A}}-\overline{\partial_{0} W^{A}}+\text { known quantities } \\
& =\nu^{0} \bar{g}^{A B} \overline{\partial_{00}^{2} g_{r B}}+\nu^{0} \bar{g}^{A B} \partial_{r} \overline{\partial_{0} g_{0 B}}-\left(\tau \nu^{0}+2 \bar{W}^{0}+2 \overline{\hat{\Gamma}}^{0}\right) \bar{g}^{A B} \overline{\partial_{0} g_{0 B}} \\
& -2 \nu^{0} \bar{g}^{B C} \overline{\partial_{0} g_{0 C}}\left(\chi_{B}{ }^{A}-\hat{\chi}_{B}{ }^{A}\right)-\overline{\partial_{0} W^{A}}+\text { known quantities . }
\end{aligned}
$$

The gauge condition $\overline{\partial_{0} H^{A}}=0$ can be used to rewrite $\bar{R}_{0 A}=0$ as a coupled linear ODE-system for $\overline{\partial_{0} g_{0 A}}$ or, depending on the setting, as an algebraic equation for $\overline{\partial_{0} W^{A}}$,

$$
\begin{array}{r}
{\left[\partial_{r}-\frac{3}{2} \tau+\frac{1}{2} \hat{\tau}-\nu_{0}\left(\bar{W}^{0}+\overline{\hat{\Gamma}}^{0}\right)\right]} \\
\overline{\partial_{0} g_{0 A}}-\left(2 \sigma_{A}^{B}-\bar{g}_{A D} \bar{g}^{B C} \hat{\sigma}_{C}{ }^{D}\right) \overline{\partial_{0} g_{0 B}} \\
=\frac{1}{2} \nu_{0} g_{A B} \overline{\partial_{0} W^{B}}+\text { known quantities }
\end{array}
$$

In the light-cone-case [2, Section 4.5] as well as in the case of two transversally intersecting null hypersurfaces the boundary condition is $\lim _{r \rightarrow 0} \overline{\partial_{0} g_{0 A}}=0$.

The gauge condition $\overline{\partial_{0} H^{A}}=0$ then determines $\overline{\partial_{00}^{2} g_{r A}}$ algebraically.

Einstein equation $\bar{R}_{00}=0$ : Finally, we have

$$
\begin{aligned}
\bar{R}_{00} & =\nu^{0}\left(\frac{1}{2} \partial_{r}+\kappa+\frac{\tau}{2}-\nu^{0} \partial_{r} \nu_{0}\right) \overline{\partial_{0} g_{00}}-\overline{\partial_{0} \Gamma_{0 r}^{r}}-\overline{\partial_{0} \Gamma_{0 A}^{A}}+\text { known quantities } \\
& =\frac{1}{2} \nu^{0} \tau \overline{\partial_{0} g_{00}}-\frac{1}{2} \bar{g}^{A B} \overline{\partial_{00}^{2} g_{A B}}+\text { known quantities } .
\end{aligned}
$$


For that calculation we used that

$$
\begin{aligned}
& \overline{\partial_{0} \Gamma_{0 r}^{r}}=-\frac{1}{2}\left(\nu^{0}\right)^{2} \overline{\partial_{0} g_{r r}} \overline{\partial_{0} g_{00}}+\frac{1}{2} \nu^{0} \partial_{r} \overline{\partial_{0} g_{00}}+\text { known quantities }, \\
& \overline{\partial_{0} \Gamma_{0 B}^{A}}=\frac{1}{2} \bar{g}^{A C} \overline{\partial_{00}^{2} g_{B C}}+\text { known quantities } .
\end{aligned}
$$

Taking into account that

$$
\overline{\partial_{0} \Gamma_{A B}^{r}}=\left(\nu^{0}\right)^{2} \chi_{A B} \overline{\partial_{0} g_{00}}-\frac{1}{2} \nu^{0} \overline{\partial_{00}^{2} g_{A B}}+\text { known quantities }
$$

we compute the $r$-component of the $u$-differentiated wave-gauge vector,

$$
\begin{aligned}
\overline{\partial_{0} H^{r}}= & \overline{\partial_{0} g^{\mu \nu}}\left(\bar{\Gamma}_{\mu \nu}^{r}-\hat{\Gamma}_{\mu \nu}^{r}\right)+\bar{g}^{\mu \nu}\left(\overline{\partial_{0} \Gamma_{\mu \nu}^{r}}-\overline{\partial_{0} \hat{\Gamma}_{\mu \nu}^{r}}\right)-\overline{\partial_{0} W^{r}} \\
= & -\left(\nu^{0}\right)^{2}\left(\kappa-\hat{\kappa}+\frac{1}{2} \nu^{0} \overline{\partial_{0} g_{r r}}\right) \overline{\partial_{0} g_{00}}+2 \nu^{0} \overline{\partial_{0} \Gamma_{0 r}^{r}}+\bar{g}^{i j} \overline{\partial_{0} \Gamma_{i j}^{r}} \\
& -\overline{\partial_{0} W^{r}}+\text { known quantities } \\
= & \left(\nu^{0}\right)^{2}\left[\partial_{r}-\frac{\tau}{2}-\kappa+\hat{\kappa}-\frac{3}{2} \nu_{0}\left(\overline{W^{0}}+\overline{\hat{\Gamma}}^{0}\right)\right] \overline{\partial_{0} g_{00}}-\frac{1}{2} \nu^{0} \bar{g}^{A B} \overline{\partial_{00}^{2} g_{A B}} \\
& -\overline{\partial_{0} W^{r}}+\text { known quantities. }
\end{aligned}
$$

Once again we exploit the gauge condition, here $\overline{\partial_{0} H^{r}}=0$, to rewrite $\bar{R}_{00}=0$ as a linear ODE for $\overline{\partial_{0} g_{00}}$, or, alternatively, an algebraic equation for $\overline{\partial_{0} W^{r}}$

$$
\left[\partial_{r}-\tau-\kappa+\hat{\kappa}-\frac{3}{2} \nu_{0}\left(\bar{W}^{0}+\overline{\hat{\Gamma}}^{0}\right)\right] \overline{\partial_{0} g_{00}}=\left(\nu_{0}\right)^{2} \overline{\partial_{0} W^{r}}+\text { known quantities . }
$$

For both light-cones [2, Section 4.5] and transversally intersecting null hypersurfaces, the boundary condition is $\lim _{r \rightarrow 0} \overline{\partial_{0} g_{00}}=0$. The gauge condition $\overline{\partial_{0} H^{r}}=0$ then determines $\bar{g}^{A B} \overline{\partial_{00}^{2} g_{A B}}$ algebraically.

The gauge scheme we want to use here now works as follows: We prescribe $\gamma, \kappa, \bar{g}_{0 \mu}$ and $\overline{\partial_{0} g_{0 \mu}}$. The Raychaudhuri equation and the vacuum Einstein wave-map gauge constraints then determine $\bar{g}_{A B}$ and $\bar{W}^{\lambda}$. We then solve the hierarchical system of equations derived above to compute $\overline{\partial_{0} W^{\lambda}}$ from (4.6), (4.7) and (4.8). We choose a smooth space-time vector field $W^{\lambda}$ which induces the computed values for $\bar{W}^{\lambda}$ and $\overline{\partial_{0} W^{\lambda}}$ on $\mathscr{N}$ (in the light-cone case it is a non-trivial issue whether such an extension off the cone exists near the tip, we return to this issue below). Then we solve the reduced Einstein equations (or the conformal field equations, cf. Section 5). It is well-known that the solution induces the prescribed data for the metric tensor on the initial surface and satisfies $H^{\lambda}=0$ with the prescribed source vector field $W^{\lambda}$, and thus solves the full Einstein equations. But then it follows from the equations (4.6)-(4.8), together with the relevant boundary conditions at the vertex or the intersection manifold, respectively, that the desired values for $\overline{\partial_{0} g_{0 \mu}}$ are induced, as well.

Some comments in the light-cone-case concerning regularity at the vertex are in order: What we have described above is exactly the strategy on two transverse characteristic surfaces (some care is needed to obtain a $W^{\lambda}$ which is continuous at the intersection manifold, cf. [6, Section 3.1]). On a null cone there is a difficulty related to the behavior near the tip: In [3] it has been shown that if the metric $\bar{g}$ is the restriction to the cone of a smooth space-time metric, then $\bar{W}^{\lambda}$ does admit a smooth extension (and the known well-posedness result 
is applicable). However, we also want to prescribe $\overline{\partial_{0} g_{0 \mu}}$, whence $\overline{\partial_{0} W^{\lambda}}$ needs to be of a specific form on the cone, as well. It seems difficult to ensure that $\bar{W}^{\lambda}$ and $\overline{\partial_{0} W^{\lambda}}$ arise from a smooth vector field $W^{\lambda}$ in space-time if one proceeds this way. To avoid dealing with the issue of extendability near the tip of the cone, the gauge scheme will be somewhat modified:

Since we are merely interested in the asymptotic behavior of the fields, it suffices for our purposes to prescribe $\bar{g}_{0 \mu}$ and $\overline{\partial_{0} g_{0 \mu}}$ just for large $r$, say $r>r_{2}$. For small $r$, say $r<r_{1}<r_{2}$ we can use a conventional scheme where for instance $\gamma, \kappa$ and $W^{\lambda}$ are prescribed with e.g. $W^{\lambda}=0$. Then the regularity issue near the tip of the cone is well-understood [3]. A smooth transition in the regime $r_{1}<r<r_{2}$ is obtained via cut-off functions:

Let $\chi \in C^{\infty}(\mathbb{R})$ be any non-negative non-increasing function satisfying $\chi(r)=0$ for $r \geq r_{2}$ and $\chi=1$ for $r \leq r_{1}$. Let $\left(\bar{g}_{0 \mu}\right)^{\circ}$ and $\left(\overline{\partial_{0} g_{0 \mu}}\right)^{\circ}$ denote the values of $\bar{g}_{0 \mu}$ and $\overline{\partial_{0} g_{0 \mu}}$ computed from $(\gamma, \kappa)$ for say $W^{\lambda}=0$, and denote by $\left(\bar{g}_{0 \mu}\right)^{\dagger}$ and $\left(\bar{\partial}_{0} g_{0 \mu}\right)^{\dagger}$ those values we want realize for $r>r_{2}$. We then set

$$
\begin{aligned}
\bar{g}_{0 \mu} & =(1-\chi)\left(\bar{g}_{0 \mu}\right)^{\dagger}+\chi\left(\bar{g}_{0 \mu}\right)^{\circ}, \\
\bar{\partial}_{0} g_{0 \mu} & =(1-\chi)\left(\overline{\partial_{0} g_{0 \mu}}\right)^{\dagger}+\chi\left(\overline{\partial_{0} g_{0 \mu}}\right)^{\circ} .
\end{aligned}
$$

For these new values for $\bar{g}_{0 \mu}$ and $\overline{\partial_{0} g_{0 \mu}}$ we go through the above scheme again. By construction we still obtain the desired values for $\bar{g}_{0 \mu}$ and $\overline{\partial_{0} g_{0 \mu}}$ for $r>$ $r_{2}$, but now we also have $\bar{W}^{\lambda}=0=\overline{\partial_{0} W^{\lambda}}$ for $r<r_{1}$, and the extension problem becomes trivial: Since there are no difficulties in extending a vector field defined along the light-cone to a neighborhood of the cone away from the tip, we conclude that $\bar{W}^{\lambda}$ and $\overline{\partial_{0} W^{\lambda}}$ arise from the restriction to the cone of some smooth vector field $W^{\lambda}$.

The coordinate $r$ which parameterizes the null rays generating the initial surface is determined to a large extent by the choice of $\kappa$. We would like to choose an $r$ for which the expansion on $\mathscr{N}$ takes the form $\tau=2 / r$. On a lightcone this is straightforward, we simply choose $\kappa=\frac{r}{2}|\sigma|^{2}$. It follows from [3] that this choice can be made up to the vertex, and it follows from the Raychaudhuri equation and regularity at the vertex that $\tau$ takes the desired form.

If $\mathscr{N}$ is one of two transversally intersecting characteristic surfaces we cannot achieve $\tau=2 / r$ globally, for the expansion needs to be regular on the intersection manifold. We would like to construct a $\kappa$ such that $\tau=2 / r$ for large $r$. Let us therefore again modify our gauge scheme slightly to bypass this issue:

Instead of $\kappa$ (supplemented by the initial data for the $\varphi$-equation $\left.\varphi\right|_{S} \neq 0$ and $\left.\partial_{r} \varphi\right|_{S}$ in the case of two characteristic surfaces, cf. [6, 10]), we prescribe the function $\varphi$ on $\mathscr{N}$. An analysis of the Raychaudhuri equation (2.5) shows that, for given $\kappa$, the existence of a nowhere vanishing $\varphi$ on $\mathscr{N}$ (which can then without loss of generality be taken to be positive), and which further satisfies $\varphi_{-1}>0$ implies $\partial_{r} \varphi>0$ on $\mathscr{N} 5$ When prescribing $\varphi$ rather than $\kappa$ we therefore assume that $\varphi$ is a strictly increasing, positive function with $\varphi_{-1}>0$. Indeed, the function $\kappa$ is then determined algebraically via the $\varphi$-equation (cf. [6] ).

In this section we have established the following setting: On $\mathscr{N}$ we regard the conformal class of the family $\gamma=\gamma_{A B} \mathrm{~d} x^{A} \mathrm{~d} x^{B}$ of Riemannian metrics as

\footnotetext{
${ }^{5}$ Indeed, we observe that for $\kappa=0$ any globally positive $\varphi$ is concave in $r$. Together with the required positivity of $\varphi_{-1}$ this implies that $\partial_{r} \varphi$ needs to be positive for all $r$. The case $\kappa \neq 0$ can be reduced to $\kappa=0$, cf. [7, Section 2.4].
} 
the "physical" initial data (in the case of two transversally intersecting null hypersurfaces supplemented by data on $\mathscr{N}_{2}$ and $S$ ), while the functions

$$
\varphi, \quad \bar{g}_{0 \mu}, \overline{\partial_{0} g_{0 \mu}},
$$

rather than $\kappa, \bar{W}^{\mu}$ and $\overline{\partial_{0} W^{\mu}}$, are regarded as gauge functions on $\mathscr{N}$, at least for $r>r_{2}$ (recall that $\varphi, \partial_{r} \varphi$ and $\nu^{0}$ need to be positive).

\subsection{Metric gauge and some conventions}

We now impose specific values for the gauge functions (4.11). Guided by their Minkowskian values we set, for $r>r_{2}$,

$$
\varphi=r, \quad \nu_{0}=1, \quad \nu_{A}=0, \quad \bar{g}_{00}=-1, \quad \overline{\partial_{0} g_{0 \mu}}=0 .
$$

Moreover, we assume a Minkowski target for $r>r_{2}$,

$$
\hat{g}=\eta \equiv-\mathrm{d} u^{2}+2 \mathrm{~d} u \mathrm{~d} r+r^{2} s_{A B} \mathrm{~d} x^{A} \mathrm{~d} x^{B} .
$$

From now on everything will be expressed in this gauge, which we call metric gauge. Although it may not explicitly be mentioned each time, since (4.12) and (4.13) are merely assumed to hold for $r>r_{2}$ we will tacitly assume henceforth that all the equations are meant to hold in this regime.

The relation between metric gauge and harmonic coordinates is discussed in Appendix B. where we address the "paradox" that in the metric gauge we do not need to impose conditions on $\gamma$ while in the harmonic gauge we do need to do it to make sure that the constraint equations admit a global solution.

We have not discussed here what in this gauge scheme (which may be used on $\mathscr{N}_{2}$ as well) the free data on the intersection manifold are, and we leave it to the reader to work this out. We are interested in the asymptotic regime, and the main object of this section was to show that the choice (4.12) can be made without any geometric restrictions for large $r$, if one does not prescribe $\kappa$ and $W^{\lambda}$ anymore, but treats them as unknowns determined by the constraints.

\subsection{Solution of the constraint equations in the metric gauge}

We solve the Einstein vacuum constraints in the metric gauge for $\kappa$ and $\bar{W}^{\lambda}$, where we assume the initial data $\gamma$ to be of the form (2.15). Recall our convention that all equations are meant to hold for $r>r_{2}$.

Equation (2.5) yields with $\varphi=r$

$$
\begin{aligned}
\kappa & =\left(\partial_{r} \varphi\right)^{-1}\left(\partial_{r r}^{2} \varphi+\frac{1}{2}|\sigma|^{2} \varphi\right)=\frac{1}{2} r|\sigma|^{2} \\
& =\frac{1}{2} \sigma_{4} r^{-3}+\mathcal{O}\left(r^{-4}\right)=\frac{1}{8}\left|\breve{h}^{(1)}\right|^{2} r^{-3}+\mathcal{O}\left(r^{-4}\right) .
\end{aligned}
$$

We further note that $\varphi=r$ implies

$$
\tau=2 / r,
$$

as desired, in particular $\tau_{2}=0$. We emphasize that the argument used for the No-Go Theorem in [7], that the coefficient $\tau_{2}$ vanishes only for Minkowski data, does not apply when $\kappa \neq 0$. In our case $\kappa$ does not vanish unless $|\sigma|^{2} \equiv 0$. 
It follows from (3.13), (4.12) and (4.15) that

$\bar{g}_{A B}=\varphi^{2} \sqrt{\frac{\operatorname{det} s}{\operatorname{det} \gamma}} \gamma_{A B}=r^{2} s_{A B}+r \breve{h}_{A B}^{(1)}+\frac{1}{4}\left|\breve{h}^{(1)}\right|^{2} s_{A B}-\left(\sigma_{A}{ }^{C}\right)_{3} s_{B C}+\mathcal{O}\left(r^{-1}\right)$.

Moreover,

$$
\begin{aligned}
\bar{W}^{0} & =-\left(2 \partial_{r}+\tau+2 \kappa\right) \nu^{0}-\overline{\hat{\Gamma}}^{0}=-2 r^{-1}-r|\sigma|^{2}+r \bar{g}^{A B} s_{A B} \\
& =\frac{1}{4}\left|\breve{h}^{(1)}\right|^{2} r^{-3}+\mathcal{O}\left(r^{-4}\right)
\end{aligned}
$$

where we used that

$$
\overline{\hat{\Gamma}}^{0}=-r \bar{g}^{A B} s_{A B}=-2 r^{-1}-\frac{1}{2}|\breve{h}(1)|^{2} r^{-3}+\mathcal{O}\left(r^{-4}\right) .
$$

The equation (2.7) which determines $\xi_{A}$ cannot be solved in explicit form. The asymptotic analysis of its solutions has already been done in Section 3.1.3 The asymptotic expansion of $\xi_{A}$ does not contain logarithmic terms if and only if the no-logs-condition is fulfilled, which in the metric gauge where $\tau_{2}=0$ reduces to

$$
\left(\sigma_{A}^{B}\right)_{3}=0 .
$$

Assuming (4.21) one finds

$$
\xi_{A}=-\stackrel{\circ}{ }^{B} \breve{h}_{A B}^{(1)} r^{-1}+C_{A}^{\left(\xi_{B}\right)}+\mathcal{O}\left(r^{-3}\right) .
$$

Now we can solve (2.8) for $\bar{W}^{A}$,

$$
\bar{W}^{A}=\xi^{A}+\bar{g}^{C D} \check{\Gamma}_{C D}^{A}-\hat{\Gamma}^{A}=\mathcal{O}\left(r^{-4}\right),
$$

where we used

$$
\hat{\Gamma}^{A}=\bar{g}^{C D} \stackrel{\circ}{\Gamma}_{C D}^{A}=s^{C D} \stackrel{\circ}{\Gamma}_{C D}^{A} r^{-2}-\breve{h}^{(1) C D} \stackrel{\circ}{\Gamma}_{C D}^{A} r^{-3}+\mathcal{O}\left(r^{-4}\right) .
$$

Note that the two leading-order terms in 4.23) cancel out.

The $\zeta$-equation (2.9) cannot be solved analytically, its asymptotic solution, which has been determined in Section 3.1.5, does not involve log terms,

$$
\zeta=-2 r^{-1}+C^{(\zeta)} r^{-2}+\mathcal{O}\left(r^{-3}\right) .
$$

Finally, we solve the constraint equation (2.10) for $\bar{W}^{r}$,

$$
\begin{aligned}
\bar{W}^{r} & =\frac{1}{2} \zeta-\left(\partial_{1}+\frac{1}{2} \tau+\kappa\right) \bar{g}^{r r}-\bar{\Gamma}^{r} \\
& =\frac{1}{2} \zeta-r^{-1}-\frac{r}{2}|\sigma|^{2}+r \bar{g}^{A B} s_{A B}=\frac{1}{2} C^{(\zeta)}+\mathcal{O}\left(r^{-3}\right),
\end{aligned}
$$

as

$$
\overline{\hat{\Gamma}}^{r}=-r \bar{g}^{A B} s_{A B}=-2 r^{-1}-\frac{1}{2}\left|\breve{h}^{(1)}\right|^{2} r^{-3}+\mathcal{O}\left(r^{-4}\right) .
$$




\subsection{Overview of the metric gauge I}

In the metric gauge one treats, at least for large $r$, some of the metric components as gauge degrees of freedom rather than $\kappa, \bar{W} \lambda$ and $\overline{\partial_{0} W^{\lambda}}$. This provides the decisive advantage that one obtains more explicit solutions of the constraint equations since most of them are algebraic equations rather than ODEs in this setting, so that the asymptotic expansions contain less integration functions, whose values are not explicitly known. Moreover, the computations of Schouten tensor, Weyl tensor etc. (as needed in Section 5 for Friedrich's equations) will be simplified significantly by the fact that several metric components take their Minkowskian values.

We have seen in Section 3 that many log terms are produced due to a bad choice of coordinates. In the metric gauge all the gauge-dependent conditions of Theorem 3.2 (cf. Remark 3.3 and compare (4.29)-(4.34) below with (3.50)(3.56) ), are satisfied and we are left with the gauge-invariant no-logs-condition, which can be expressed as an explicit condition on $\gamma$ in this gauge,

$$
0=\left(\sigma_{A}^{B}\right)_{3}=\frac{1}{2} h^{(1)} \breve{h}_{A}^{(1) B}-\breve{h}_{A}^{(2) B} .
$$

Consequently we can freely prescribe all the $h_{A B}^{(n)}$ 's except for $\breve{h}_{A B}^{(2)}$ which is determined by (4.28).

It is easy to find sufficient conditions on the initial data $\gamma$ such that the nologs-condition (3.49) in its general form is fulfilled (just take $\breve{h}_{A B}^{(1)}=\breve{h}_{A B}^{(2)}=0$ ), i.e. Theorem 3.2 shows that there is a large class of initial data for which the metric admits a smooth conformal completion at infinity. To find necessary conditions on $\gamma$ is more involved, since the expansion coefficient $\tau_{2}$ is in general not explicitly known. In the metric gauge, though, $\tau_{2}$ is explicitly known, whence it is easily possible to characterize the initial data sets completely which lead to the restriction of a metric to $\mathscr{N}$ which admits a smooth conformal completion at infinity. Moreover, it becomes obvious that for "generic" initial data the no-logs-condition will be violated.

Note that we have not studied yet the asymptotic behavior of the unknowns of the conformal field equations which also involve transverse derivatives of the metric. The gauge functions are more complicated in the metric gauge and contain certain integration functions, and one might wonder whether log-terms arise at some later stage. Luckily, it turns out (cf. Section 5) that this is not the case, and that the no-logs-condition is necessary-and-sufficient to guarantee smoothness of all the relevant fields at conformal infinity .

Of course, once we have determined the gauge functions it is possible to return to the original viewpoint and regard them as the relevant gauge degrees of freedom. This leads to an initial data-dependent gauge. Assuming that the initial data $\gamma$ satisfy the no-logs-condition (4.28) we give their values for $r>r_{2}$ (the functions $\overline{\partial_{0} W^{\lambda}}$ will be computed in Section 4.6):

$$
\begin{aligned}
\kappa & =\frac{1}{2} r|\sigma|^{2}=\frac{1}{8}\left|\breve{h}^{(1)}\right|^{2} r^{-3}+\mathcal{O}\left(r^{-4}\right) \\
\bar{W}^{0} & =-r|\sigma|^{2}-\frac{2}{r}-\overline{\hat{\Gamma}}^{0}=\frac{1}{4}\left|\breve{h}^{(1)}\right|^{2} r^{-3}+\mathcal{O}\left(r^{-4}\right) \\
\bar{W}^{A} & =\xi^{A}+\bar{g}^{C D} \check{\Gamma}_{C D}^{A}-\hat{\Gamma}^{A}=\mathcal{O}\left(r^{-4}\right) \\
\bar{W}^{r} & =\frac{1}{2} \zeta-r^{-1}-\frac{1}{2} r|\sigma|^{2}-\overline{\hat{\Gamma}}^{r}=\mathcal{O}\left(r^{-2}\right)
\end{aligned}
$$


with $\xi_{A}$ and $\zeta$ given by (4.22) and (4.25), respectively. The restrictions to $\mathscr{N}$ of the metric components then take the form (again for $r>r_{2}$ ):

$$
\begin{gathered}
\bar{g}_{00}=-1, \quad \nu_{0}=1, \quad \nu_{A}=0, \quad \bar{g}_{r r}=\bar{g}_{r A}=0, \\
\bar{g}_{A B}=r^{2} s_{A B}+r \breve{h}_{A B}^{(1)}+\frac{1}{4}\left|\breve{h}^{(1)}\right|^{2} s_{A B}+\mathcal{O}\left(r^{-1}\right) .
\end{gathered}
$$

REMARK 4.1 The asymptotic expansion of $\bar{g}_{A B}$ depends only on the gauge choice for $\kappa$. Our $\kappa$, equation (4.29), coincides with the $\kappa$ used to define Bondi coordinates (where one also requires $\tau=2 / r$ ). The no-logs-condition which, in a $\kappa=\frac{r}{2}|\sigma|^{2}$-gauge is equivalent to the absence of $s$-trace-free terms in $\left(\bar{g}_{A B}\right)_{0}$,

$$
\left(\bar{g}_{A B}\right)_{0}=0
$$

recovers the "outgoing wave condition" imposed a priori by Bondi et al. and by Sachs to inhibit the appearance of logarithmic terms (cf. e.g. [5]).

For an arbitrary $\kappa=\mathcal{O}\left(r^{-3}\right)$ the correct generalization of this condition, equivalent to the no-logs-condition (3.49), is (cf. (3.13)),

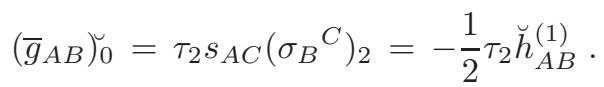

\subsection{Transverse derivatives of the metric on $\mathscr{N}$}

In this section we compute the $\overline{\partial_{0} W^{\lambda}}$ 's and certain transverse derivatives of the metric on $\mathscr{N}$ (the asymptotic expansions thereof) in the metric gauge using the vacuum Einstein equations $R_{\mu \nu}=0$ and assuming that the no-logs-condition is fulfilled, so that (4.29)-(4.34) and $\overline{\partial_{0} g_{0 \mu}}=0$ hold. As before, all equalities are meant to be valid for $r>r_{2}$, even if this is not mentioned wherever relevant.

It follows from (4.1)-(4.3) that

$$
\begin{aligned}
\overline{\partial_{0} g_{r r}} & =-r|\sigma|^{2}=-2 \kappa, \\
\bar{\partial}_{0} g_{r A} & =\xi_{A}, \\
\bar{g}^{A B} \frac{\partial_{0} g_{A B}}{} & =-\zeta-\tau .
\end{aligned}
$$

If we plug in the values for $\bar{g}_{\mu \nu}$ and $\overline{\partial_{0} g_{\mu \nu}}$ we find from [2, Appendix A] the following expressions for the Christoffel symbols restricted to $\mathscr{N}$, which we shall made frequently use of:

$$
\begin{gathered}
\bar{\Gamma}_{00}^{\mu}=\bar{\Gamma}_{r r}^{0}=\bar{\Gamma}_{r A}^{0}=\bar{\Gamma}_{r r}^{C}=0, \bar{\Gamma}_{r r}^{r}=-\bar{\Gamma}_{0 r}^{0}=-\bar{\Gamma}_{0 r}^{r}=\kappa \\
\bar{\Gamma}_{0 A}^{0}=\bar{\Gamma}_{0 A}^{r}=-\bar{\Gamma}_{r A}^{r}=\frac{1}{2} \xi_{A}, \quad \bar{\Gamma}_{0 r}^{C}=\frac{1}{2} \xi^{C}, \bar{\Gamma}_{r A}^{C}=\chi_{A}^{C}, \bar{\Gamma}_{A B}^{0}=-\chi_{A B}, \\
\bar{\Gamma}_{A B}^{r}=-\frac{1}{2} \bar{\partial}_{0} g_{A B}-\chi_{A B}, \quad \bar{\Gamma}_{0 A}^{C}=\frac{1}{2} \bar{g}^{C D} \frac{\partial_{0} g_{A D}}{\partial_{A B}}, \bar{\Gamma}_{A B}^{C}=\check{\Gamma}_{A B}^{C} .
\end{gathered}
$$

Einstein equations $\breve{\bar{R}}_{A B}=0$ : From (4.4) we obtain

$$
\begin{aligned}
\bar{R}_{A B}= & \check{R}_{A B}+\overline{\partial_{0} \Gamma_{A B}^{0}}+\left(\partial_{r}+\bar{\Gamma}_{r C}^{C}\right) \bar{\Gamma}_{A B}^{r}+\bar{\Gamma}_{A B}^{0}\left(\bar{\Gamma}_{00}^{0}+\bar{\Gamma}_{0 r}^{r}+\bar{\Gamma}_{0 C}^{C}\right) \\
& -2 \bar{\Gamma}_{0(A}^{C} \bar{\Gamma}_{B) C}^{0}-2 \bar{\Gamma}_{r A}^{r} \bar{\Gamma}_{r B}^{r}-2 \bar{\Gamma}_{r(A}^{C} \bar{\Gamma}_{B) C}^{r} .
\end{aligned}
$$

First of all we have to determine the $u$-differentiated Christoffel symbol,

$$
\left.\overline{\partial_{0} \Gamma_{A B}^{0}}=-\left(\frac{1}{2} \partial_{r}+\kappa\right) \overline{\partial_{0} g_{A B}}-2 \kappa \chi_{A B}+\check{\nabla}_{(A} \xi_{B}\right) .
$$


Employing (4.38)-(4.40) and the relation $\check{R}_{A B}=\frac{1}{2} \check{R} \bar{g}_{A B}$ we obtain after some simplifications that

$$
\begin{aligned}
& \breve{R}_{A B}=-\left(\partial_{r}-\frac{1}{r}+\frac{r}{2}|\sigma|^{2}\right)\left(\overline{\partial_{0} g_{A B}}\right)+2 \sigma_{(A}^{C}\left(\overline{\partial_{|0|} g_{B) C}}\right)-\left(\partial_{r} \sigma_{A B}\right) \\
& \left.+\left(\frac{\zeta}{2}+\frac{1}{r}-\frac{r}{2}|\sigma|^{2}\right) \sigma_{A B}-\frac{1}{2}\left(\xi_{A} \xi_{B}\right)^{\swarrow}+\left(\check{\nabla}_{(A} \xi_{B}\right)\right)^{\zeta},
\end{aligned}
$$

which should vanish in vacuum. The equations $\breve{R}_{A B}=0$ form a closed linear ODE-system for $\left(\overline{\partial_{0} g_{A B}}\right)$. Since it is generally hopeless to look for an analytic solution, we content ourselves with computing the asymptotic solution. For this it is convenient to rewrite the matrix equation (4.42) as a vector equation,

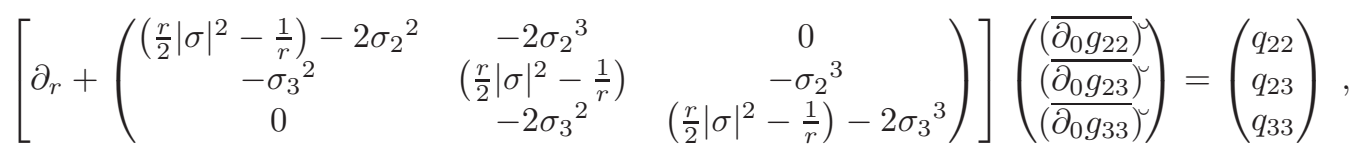

where

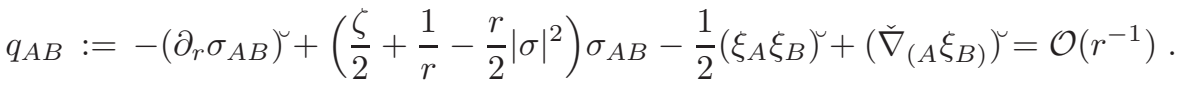

In order to permit an easier comparison with Appendix $\mathrm{A}$. we apply the transformation $r \mapsto x:=1 / r$ (with all quantities treated as scalars). Then the ODE adopts the following asymptotic form,

$$
\left[x \partial_{x}+\left(\begin{array}{lll}
1 & 0 & 0 \\
0 & 1 & 0 \\
0 & 0 & 1
\end{array}\right)+M\right]\left(\frac{\left(\overline{\partial_{0} g_{22}}\right)}{\left(\overline{\partial_{0} g_{23}}\right)}\right)=\mathcal{O}(1),
$$

where $M=\operatorname{Mat}(3,3)=\mathcal{O}(x)$. In the notation of Appendix $₫$ we observe that $\lambda=-1$ and $\hat{\ell}=-1$, and no logarithmic terms appear in the asymptotic solution. Moreover, it is $\mathcal{O}(r)$ and the integration functions are represented by the leading order terms,

$$
\left(\overline{\partial_{0} g_{A B}}\right)=D_{A B} r+\mathcal{O}(1) .
$$

Note that $D_{A B}$ is symmetric, $D_{A B}=D_{B A}$, and $s$-trace-free, $s^{A B} D_{A B}=0$.

Einstein equation $\bar{R}_{0 r}=0$ :

$\bar{R}_{0 r}=\partial_{r} \bar{\Gamma}_{0 r}^{r}+\check{\nabla}_{A} \bar{\Gamma}_{0 r}^{A}-\overline{\partial_{0} \Gamma_{r r}^{r}}-\overline{\partial_{0} \Gamma_{r A}^{A}}+\bar{\Gamma}_{0 r}^{0} \bar{\Gamma}_{0 A}^{A}+\bar{\Gamma}_{0 r}^{r}\left(\bar{\Gamma}_{0 r}^{0}+\bar{\Gamma}_{r A}^{A}\right)-\bar{\Gamma}_{r B}^{A} \bar{\Gamma}_{0 A}^{B}$.

We determine the $u$-differentiated Christoffel symbols involved,

$$
\begin{aligned}
\overline{\partial_{0} \Gamma_{r r}^{r}} & =-\frac{1}{2} \overline{\partial_{00}^{2} g_{r r}}+\frac{r^{2}}{2}|\sigma|^{4}-\frac{1}{2}|\sigma|^{2}-\frac{r}{2} \partial_{r}|\sigma|^{2}, \\
\overline{\partial_{0} \Gamma_{r A}^{C}} & =\frac{1}{2} \xi_{A} \xi^{C}+\frac{1}{2} \partial_{r}\left(\bar{g}{ }^{B C} \overline{\partial_{0} g_{A B}}\right)+\bar{g}^{B C} \check{\nabla}_{[A} \xi_{B]} \\
\Longrightarrow \overline{\partial_{0} \Gamma_{r A}^{A}} & =\frac{1}{2} \xi_{A} \xi^{A}-\frac{1}{2} \partial_{r} \zeta+\frac{1}{r^{2}} .
\end{aligned}
$$


Note that $\overline{\partial_{00}^{2} g_{r r}}$ is the only unknown at this stage. We obtain

$$
\begin{aligned}
\bar{R}_{0 r}= & \frac{1}{2} \overline{\partial_{00}^{2} g_{r r}}+\frac{1}{2}\left(\partial_{r}+r^{-1}+\frac{r}{2}|\sigma|^{2}\right) \zeta-\frac{r^{2}}{4}|\sigma|^{4}-\frac{1}{2}|\sigma|^{2} \\
& +\frac{1}{2}\left(\check{\nabla}_{A}-\xi_{A}\right) \xi^{A}-\frac{1}{2} \sigma^{A B} \overline{\partial_{0} g_{A B}},
\end{aligned}
$$

which, again, should vanish in vacuum. With (2.9) that yields

$$
\overline{\partial_{00}^{2} g_{r r}}=\frac{1}{r} \zeta+\check{R}+\frac{1}{2} \xi_{A} \xi^{A}+\frac{r^{2}}{2}|\sigma|^{4}+|\sigma|^{2}+\sigma^{A B} \overline{\partial_{0} g_{A B}}=\mathcal{O}\left(r^{-3}\right) .
$$

The leading-order term

$$
\Xi:=\left(\overline{\partial_{00}^{2} g_{r r}}\right)_{3}=\zeta_{2}+\left[D_{A}^{B}-2 \stackrel{\circ}{\nabla}_{A} \stackrel{\circ}{\nabla}^{B}\right]\left(\sigma_{B}{ }^{A}\right)_{2} .
$$

depends on certain integration functions and is not explicitly known. We choose this special notation, though, since it will appear several times in the leadingorder terms of other expansions.

The gauge condition then provides an algebraic equation for $\overline{\partial_{0} W^{0}}$,

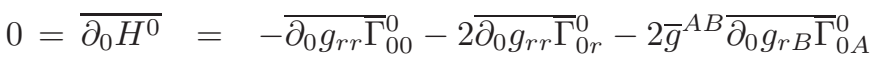

$$
\begin{aligned}
& +\overline{\partial_{0} g^{A B}}\left(\bar{\Gamma}_{A B}^{0}+r s_{A B}\right)+2 \overline{\partial_{0} \Gamma_{0 r}^{0}}+\overline{\partial_{0} \Gamma_{r r}^{0}}+\bar{g}^{A B} \overline{\partial_{0} \Gamma_{A B}^{0}}-\overline{\partial_{0} W^{0}} \\
& =\overline{\partial_{00}^{2} g_{r r}}+r s_{A B} \overline{\partial_{0} g^{A B}}+\check{\nabla}_{A} \xi^{A}-2 \xi_{A} \xi^{A}-\frac{1}{r^{2}}-\frac{3}{2} r^{2}|\sigma|^{4} \\
& -\frac{3}{2}|\sigma|^{2}-\frac{r}{2} \partial_{r}|\sigma|^{2}+\frac{1}{2}\left(\partial_{r}+r|\sigma|^{2}\right) \zeta-\overline{\partial_{0} W^{0}},
\end{aligned}
$$

where we used that

$$
\begin{aligned}
& \overline{\partial_{0} \Gamma_{r r}^{0}}=\frac{r^{2}}{2}|\sigma|^{4}-\frac{1}{2}|\sigma|^{2}-\frac{r}{2} \partial_{r}|\sigma|^{2}, \\
& \overline{\partial_{0} \Gamma_{0 r}^{0}}=\frac{1}{2} \overline{\partial_{00}^{2} g_{r r}}-\frac{r^{2}}{2}|\sigma|^{4}-\frac{1}{2} \xi_{A} \xi^{A} .
\end{aligned}
$$

Inserting (4.45) and using again (2.9) we deduce that

$$
\begin{aligned}
\overline{\partial_{0} W^{0}}= & -\left(\frac{1}{2} \partial_{r}+\frac{1}{r}\right) \zeta+\left(r s_{A B}-\sigma_{A B}\right) \overline{\partial_{0} g^{A B}}-\frac{1}{r^{2}}-\frac{r}{2} \partial_{r}|\sigma|^{2} \\
& -\frac{1}{2}|\sigma|^{2}-r^{2}|\sigma|^{4}-\xi_{A} \xi^{A} \\
= & \mathcal{O}\left(r^{-3}\right) .
\end{aligned}
$$

Einstein equations $\bar{R}_{0 A}=0$ :

$$
\begin{aligned}
\bar{R}_{0 A}= & \partial_{r} \bar{\Gamma}_{0 A}^{r}+\check{\nabla}_{B} \bar{\Gamma}_{0 A}^{B}-\overline{\partial_{0} \Gamma_{r A}^{r}}-\overline{\partial_{0} \Gamma_{A B}^{B}}+\bar{\Gamma}_{0 A}^{0} \bar{\Gamma}_{0 B}^{B}+\left(\bar{\Gamma}_{r r}^{r}+\bar{\Gamma}_{r B}^{B}\right) \bar{\Gamma}_{0 A}^{r} \\
& +\bar{\Gamma}_{0 A}^{B} \bar{\Gamma}_{r B}^{r}-\bar{\Gamma}_{A B}^{0} \bar{\Gamma}_{00}^{B}-2 \bar{\Gamma}_{r A}^{r} \bar{\Gamma}_{0 r}^{r}-\bar{\Gamma}_{A B}^{r} \bar{\Gamma}_{0 r}^{B}-\bar{\Gamma}_{r A}^{B} \bar{\Gamma}_{0 B}^{r} .
\end{aligned}
$$

For the $u$-differentiated Christoffel symbols we find,

$$
\begin{aligned}
\overline{\partial_{0} \Gamma_{r A}^{r}} & =-\frac{1}{2} \overline{\partial_{00}^{2} g_{r A}}-\chi_{A}^{B} \xi_{B}-\frac{r}{2} \partial_{A}|\sigma|^{2}-\frac{1}{2} r|\sigma|^{2} \xi_{A} \\
\overline{\partial_{0} \Gamma_{A B}^{C}} & =\bar{g}^{C D} \check{\nabla}_{(A} \overline{\partial_{|0|} g_{B) D}}-\frac{1}{2} \check{\nabla}^{C} \overline{\partial_{0} g_{A B}}+\frac{1}{2} \xi^{C} \overline{\partial_{0} g_{A B}}+\xi^{C} \chi_{A B} \\
\Longrightarrow \overline{\partial_{0} \Gamma_{A B}^{B}} & =\chi_{A}{ }^{B} \xi_{B}+\frac{1}{2} \xi^{B} \overline{\partial_{0} g_{A B}}-\frac{1}{2} \partial_{A} \zeta .
\end{aligned}
$$


We insert these expressions into the formula for $\bar{R}_{0 A}$ to obtain,

$$
\begin{aligned}
\bar{R}_{0 A}= & \frac{1}{2}\left(\partial_{r}+r^{-1}\right) \xi_{A}+\frac{1}{2}\left(\check{\nabla}^{B}-\xi^{B}\right) \overline{\partial_{0} g_{A B}}+\frac{r}{2}\left(\partial_{A}+\frac{1}{2} \xi_{A}\right)|\sigma|^{2}+\frac{1}{2} \overline{\partial_{00}^{2} g_{r A}} \\
& +\frac{1}{2}\left(\partial_{A}-\frac{1}{2} \xi_{A}\right) \zeta
\end{aligned}
$$

which should vanish in vacuum, i.e.

$$
\begin{aligned}
\overline{\partial_{00}^{2} g_{r A}} & =\left(\xi^{B}-\check{\nabla}^{B}\right) \overline{\partial_{0} g_{A B}}-\left(\partial_{r}+\frac{1}{r}-\frac{\zeta}{2}\right) \xi_{A}-r\left(\partial_{A}+\frac{\xi_{A}}{2}\right)|\sigma|^{2}-\partial_{A} \zeta(4 . \\
& =-\check{\nabla}_{B} D_{A}{ }^{B} r^{-1}+\mathcal{O}\left(r^{-2}\right) .
\end{aligned}
$$

We employ the gauge condition $\overline{\partial_{0} H^{A}}=0$ to compute $\overline{\partial_{0} W^{A}}$. This requires the knowledge of further $u$-differentiated Christoffel symbols,

$$
\begin{aligned}
& \overline{\partial_{0} \Gamma_{0 r}^{C}}=\frac{1}{2} \bar{g}^{C D} \overline{\partial_{00}^{2} g_{r D}}+\frac{r}{2}|\sigma|^{2} \xi^{C}+\frac{1}{2} \xi_{D} \overline{\partial_{0} g^{C D}} \\
& \overline{\partial_{0} \Gamma_{r r}^{C}}=\bar{g}^{C D} \partial_{r} \xi_{D}-\frac{1}{2} r|\sigma|^{2} \xi^{C}+\frac{r}{2} \check{\nabla}^{C}|\sigma|^{2} .
\end{aligned}
$$

We then obtain

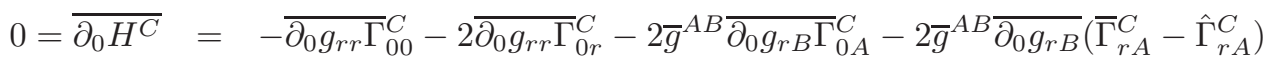

$$
\begin{aligned}
& +\overline{\partial_{0} g^{A B}}\left(\bar{\Gamma}_{A B}^{C}-\hat{\Gamma}_{A B}^{C}\right)+2 \overline{\partial_{0} \Gamma_{0 r}^{C}}+\overline{\partial_{0} \Gamma_{r r}^{C}}+\bar{g}^{A B} \overline{\partial_{0} \Gamma_{A B}^{C}}-\overline{\partial_{0} W^{C}} \\
& =\left(\partial_{r}+\frac{3}{r}+\frac{3}{2} r|\sigma|^{2}\right) \xi^{C}+\bar{g}^{C D} \overline{\partial_{00}^{2} g_{r D}}+\frac{1}{2}\left(\check{\nabla}^{C}-\xi^{C}\right) \zeta+\frac{r}{2} \check{\nabla}^{C}|\sigma|^{2} \\
& +\overline{\partial_{0} g^{A B}}\left(\check{\Gamma}_{A B}^{C}-\stackrel{\circ}{\Gamma}_{A B}^{C}\right)-\left(\check{\nabla}_{A}-2 \xi_{A}\right) \overline{\partial_{0} g^{A C}}-\overline{\partial_{0} W^{C}} \text {. }
\end{aligned}
$$

Combining this with the expression we found for $\overline{\partial_{00}^{2} g_{r A}}$ yields

$$
\begin{aligned}
\overline{\partial_{0} W^{A}}= & \left(\overline{\partial_{0} g^{A B}}-2 \sigma^{A B}\right) \xi_{B}-\frac{r}{2}\left(\check{\nabla}^{A}-2 \xi^{A}\right)|\sigma|^{2}-\frac{1}{2} \check{\nabla}^{A} \zeta \\
& +\overline{\partial_{0} g^{B C}}\left(\check{\Gamma}_{B C}^{A}-\stackrel{\circ}{\Gamma}_{B C}^{A}\right) \\
= & \mathcal{O}\left(r^{-4}\right) .
\end{aligned}
$$

Einstein equation $\bar{R}_{00}=0$ : The (00)-component of the Ricci tensor satisfies

$$
\bar{R}_{00}=\left(\partial_{r}+2 \bar{\Gamma}_{r r}^{r}+\bar{\Gamma}_{r A}^{A}\right) \bar{\Gamma}_{00}^{r}-\overline{\partial_{0} \Gamma_{0 r}^{r}}-\overline{\partial_{0} \Gamma_{0 A}^{A}}-\bar{\Gamma}_{0 r}^{r} \bar{\Gamma}_{0 r}^{r}-2 \bar{\Gamma}_{0 A}^{r} \bar{\Gamma}_{0 r}^{A}-\bar{\Gamma}_{0 B}^{A} \bar{\Gamma}_{0 A}^{B} .
$$

The $u$-differentiated Christoffel symbols appearing in this expression satisfy,

$$
\begin{aligned}
\overline{\partial_{0} \Gamma_{0 r}^{r}} & =\frac{1}{2 r} \zeta+\frac{1}{2} \check{R}-\frac{1}{4} \xi_{A} \xi^{A}-\frac{r^{2}}{4}|\sigma|^{4}+\frac{1}{2}|\sigma|^{2}+\frac{1}{2} \sigma^{A B} \overline{\partial_{0} g_{A B}} \\
\overline{\partial_{0} \Gamma_{0 A}^{A}} & =\frac{1}{2} \bar{g}^{A B} \overline{\partial_{00}^{2} g_{A B}}+\frac{1}{2} \overline{\partial_{0} g^{A B}} \overline{\partial_{0} g_{A B}}-\frac{1}{2} \xi_{A} \xi^{A}
\end{aligned}
$$

and we are led to

$$
\bar{R}_{00}=-\frac{1}{2} \bar{g}^{A B} \overline{\partial_{00}^{2} g_{A B}}-\left(\frac{1}{2} \sigma^{A B}+\frac{1}{4} \overline{\partial_{0} g^{A B}}\right) \overline{\partial_{0} g_{A B}}-\frac{1}{2 r} \zeta-\frac{1}{2} \check{R}+\frac{1}{4} \xi_{A} \xi^{A}-\frac{1}{2}|\sigma|^{2},
$$


which should vanish in vacuum, solving for $\bar{g}^{A B} \overline{\partial_{00}^{2} g_{A B}}$ we are led to

$$
\begin{aligned}
\bar{g}^{A B} \overline{\partial_{00}^{2} g_{A B}} & =-\left(\sigma^{A B}+\frac{1}{2} \overline{\partial_{0} g^{A B}}\right) \overline{\partial_{0} g_{A B}}-\frac{1}{r} \zeta-\check{R}+\frac{1}{2} \xi_{A} \xi^{A}-|\sigma|^{2} \\
& =\frac{1}{2}|D|^{2} r^{-2}+\mathcal{O}\left(r^{-3}\right) .
\end{aligned}
$$

To compute $\overline{\partial_{0} W^{r}}$ we employ the gauge condition

$$
\begin{aligned}
0=\overline{\partial_{0} H^{r}}=- & \overline{\partial_{0} g_{r r}}\left(\bar{\Gamma}_{00}^{r}-\bar{\Gamma}_{r r}^{r}\right)-2 \bar{g}^{A B} \overline{\partial_{0} g_{r B} \Gamma_{0 A}^{r}}-2 \bar{g}^{A B} \bar{\partial}_{0} g_{r B} \bar{\Gamma}_{r A}^{r} \\
& +\overline{\partial_{0} g^{A B}}\left(\bar{\Gamma}_{A B}^{r}-\hat{\Gamma}_{A B}^{r}\right)+2 \overline{\partial_{0} \Gamma_{0 r}^{r}}+\overline{\partial_{0} \Gamma_{r r}^{r}}+\bar{g}^{A B} \overline{\partial_{0} \Gamma_{A B}^{r}}-\overline{\partial_{0} W^{r}} \\
= & \frac{1}{2}\left(\check{\nabla}_{A}-\xi_{A}\right) \xi^{A}-\frac{1}{2} \bar{g}^{A B} \overline{\partial_{00}^{2} g_{A B}}-\frac{1}{r^{2}}-\left(1+\frac{r}{2} \partial_{r}\right)|\sigma|^{2}-\frac{3}{4} r^{2}|\sigma|^{4} \\
& -\frac{\zeta}{2}\left(\frac{1}{r}-\frac{r}{2}|\sigma|^{2}\right)+\left(r s_{A B}-\frac{1}{2} \sigma_{A B}-\frac{1}{2} \overline{\partial_{0} g_{A B}}\right) \overline{\partial_{0} g^{A B}}-\overline{\partial_{0} W^{r}(4.62)}
\end{aligned}
$$

For this calculation we used that

$\bar{g}^{A B} \overline{\partial_{0} \Gamma_{A B}^{r}}=\check{\nabla}_{A} \xi^{A}+\frac{1}{2}\left(\partial_{r}+\frac{2}{r}+r|\sigma|^{2}\right) \zeta+\frac{1}{r^{2}}-|\sigma|^{2}-\sigma^{A B} \overline{\partial_{0} g_{A B}}-\frac{1}{2} \bar{g}^{A B} \overline{\partial_{00}^{2} g_{A B}}$.

We solve (4.62) for $\overline{\partial_{0} W^{r}}$, and insert the expression we found for $\bar{g}^{A B} \overline{\partial_{00}^{2} g_{A B}}$,

$$
\begin{aligned}
\overline{\partial_{0} W^{r}}= & \frac{1}{2}\left(\check{\nabla}_{A}-\frac{3}{2} \xi_{A}\right) \xi^{A}+\frac{1}{4} r|\sigma|^{2} \zeta+\frac{1}{2} \check{R}-\frac{1}{r^{2}}-\frac{1}{2}|\sigma|^{2}-\frac{r}{2} \partial_{r}|\sigma|^{2} \\
& -\frac{3}{4} r^{2}|\sigma|^{4}-\left(\frac{1}{4} \overline{\partial_{0} g_{A B}}+\sigma_{A B}-r s_{A B}\right) \overline{\partial_{0} g^{A B}} \\
= & \frac{1}{4}|D|^{2} r^{-2}+\mathcal{O}\left(r^{-3}\right) .
\end{aligned}
$$

Einstein equations $\left(\overline{\partial_{0} R_{A B}}\right)=0$ Assuming the gauge condition $H^{\lambda}=0$ the Einstein equations $\left(\overline{\partial_{0} R_{A B}}\right)=0$ provide a linear ODE-system for $\left(\overline{\partial_{00}^{2} g_{A B}}\right)$. We have

$\overline{\partial_{0} R_{A B}}=\overline{\partial_{\lambda} \partial_{0} \Gamma_{A B}^{\lambda}}-\partial_{A} \overline{\partial_{0} \Gamma_{\lambda B}^{\lambda}}+\bar{\Gamma}_{\rho \lambda}^{\lambda} \overline{\partial_{0} \Gamma_{A B}^{\rho}}+\bar{\Gamma}_{A B}^{\rho} \overline{\partial_{0} \Gamma_{\rho \lambda}^{\lambda}}-\bar{\Gamma}_{\rho A}^{\lambda} \overline{\partial_{0} \Gamma_{\lambda B}^{\rho}}-\bar{\Gamma}_{\lambda B}^{\rho} \overline{\partial_{0} \Gamma_{\rho A}^{\lambda}}$.

The right-hand side contains several first-order $u$-differentiated Christoffel symbols and one of second-order, $\overline{\partial_{00}^{2} \Gamma_{A B}^{0}}$, which we have not determined yet,

$$
\begin{aligned}
& \overline{\partial_{0} \Gamma_{00}^{0}}=\overline{\partial_{00}^{2} g_{0 r}}, \\
& \overline{\partial_{0} \Gamma_{0 A}^{0}}=\frac{1}{2} \overline{\partial_{00}^{2} g_{r A}}-\frac{1}{2} \xi^{B} \overline{\partial_{0} g_{A B}}+\frac{r}{2}|\sigma|^{2} \xi_{A} \\
& =-\frac{1}{2} \stackrel{\circ}{\nabla}_{B} D_{A}^{B} r^{-1}+\mathcal{O}\left(r^{-2}\right) \text {, } \\
& \overline{\partial_{0} \Gamma_{r A}^{0}}=-\chi_{A}{ }^{B} \xi_{B}-\frac{r}{2}\left(\partial_{A}+\xi_{A}\right)|\sigma|^{2} \\
& =-2 \stackrel{\circ}{\nabla}_{B}\left(\sigma_{A}^{B}\right)_{2} r^{-2}+\mathcal{O}\left(r^{-3}\right) \text {, } \\
& \overline{\partial_{0} \Gamma_{A B}^{r}}=-\frac{1}{2} \overline{\partial_{00}^{2} g_{A B}}-\frac{1}{2}\left(\partial_{r}+r|\sigma|^{2}\right) \overline{\partial_{0} g_{A B}}+\check{\nabla}_{(A} \xi_{B)}-r|\sigma|^{2} \chi_{A B}(4.70) \\
& =-\frac{1}{2} \overline{\partial_{00}^{2} g_{A B}}-\frac{1}{2} D_{A B}+\mathcal{O}\left(r^{-1}\right), \\
& \overline{\partial_{0} \Gamma_{0 A}^{C}}=-\frac{1}{2} \xi_{A} \xi^{C}+\frac{1}{2} \overline{\partial_{0} g^{C D}} \overline{\partial_{0} g_{A D}}+\frac{1}{2} \bar{g}^{C D} \overline{\partial_{00}^{2} g_{A D}} \\
& =\frac{1}{2} g^{C D} \partial_{00}^{2} g_{A D}-\frac{1}{2} D_{A D} D^{C D} r^{-2}+\mathcal{O}\left(r^{-3}\right) \text {. }
\end{aligned}
$$


To calculate the two-times $u$-differentiated Christoffel symbol we use that

$$
\overline{\partial_{00}^{2} g^{\mu \nu}}=-2 \bar{g}^{\nu \sigma} \overline{\partial_{0} g^{\mu \rho} \partial_{0} g_{\rho \sigma}}-\bar{g}^{\mu \rho} \bar{g}^{\nu \sigma} \overline{\partial_{00}^{2} g_{\rho \sigma}},
$$

whence

$$
\begin{aligned}
\overline{\partial_{00}^{2} g^{00}} & =-\overline{\partial_{00}^{2} g_{r r}}+2 \xi_{A} \xi^{A}+2 r^{2}|\sigma|^{4} \\
& =-\Xi r^{-3}+\mathcal{O}\left(r^{-4}\right) \\
\overline{\partial_{00}^{2} g^{0 r}} & =-\overline{\partial_{00}^{2} g_{0 r}}-\overline{\partial_{00}^{2} g_{r r}}+\underbrace{2 \xi_{A} \xi^{A}+2 r^{2}|\sigma|^{4}}_{=\mathcal{O}\left(r^{-4}\right)}, \\
\overline{\partial_{00}^{2} g^{0 C}} & =-\bar{g}^{C D} \overline{\partial_{00}^{2} g_{r D}}-2 r|\sigma|^{2} \xi^{C}-2 \xi_{D} \overline{\partial_{0} g^{C D}} \\
& =\dot{\circ}_{B} D^{B C} r^{-3}+\mathcal{O}\left(r^{-4}\right) .
\end{aligned}
$$

That yields

$$
\begin{aligned}
\overline{\partial_{00}^{2} \Gamma_{A B}^{0}}= & \left(\frac{1}{2} \overline{\partial_{00}^{2} g_{r r}}-r^{2}|\sigma|^{4}-\xi_{C} \xi^{C}\right)\left(\overline{\partial_{0} g_{A B}}+2 \chi_{A B}\right)+\chi_{A B} \overline{\partial_{00}^{2} g_{0 r}} \\
& -\left(\frac{1}{2} \partial_{r}+r|\sigma|^{2}\right) \overline{\partial_{00}^{2} g_{A B}}-r|\sigma|^{2} \partial_{r} \overline{\partial_{0} g_{A B}}+2 r|\sigma|^{2} \check{\nabla}_{(A} \xi_{B)} \\
& -\xi^{C}\left(2 \check{\nabla}_{(A} \overline{\partial_{|0|} g_{B) C}}-\check{\nabla}_{C} \overline{\partial_{0} g_{A B}}\right)+\check{\nabla}_{(A} \overline{\partial_{|00|}^{2} g_{B) r}} \\
= & \chi_{A B} \overline{\partial_{00}^{2} g_{0 r}}-\left(\frac{1}{2} \partial_{r}+r|\sigma|^{2}\right) \overline{\partial_{00}^{2} g_{A B}}-\stackrel{\circ}{\nabla}_{(A} \stackrel{\circ}{\nabla}_{|C|} D_{B)}{ }^{C} r^{-1}+\mathcal{O}\left(r^{-2}\right) .
\end{aligned}
$$

Now all quantities have been determined which are needed to compute $\left(\overline{\partial_{0} R_{A B}}\right)$ (the $\overline{\partial_{00}^{2} g_{0 r}}$ 's cancel out); we are just interested in the asymptotic behavior,

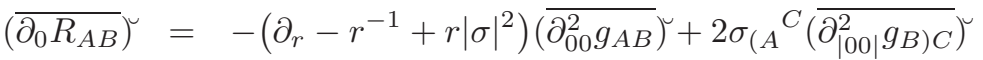

$$
\begin{aligned}
& -\frac{1}{2}\left(\Delta_{s}-2\right) D_{A B} r^{-1}+\mathcal{O}\left(r^{-2}\right) \text {, }
\end{aligned}
$$

and this expression should vanish in vacuum.

We need to check whether there are logarithmic terms in the asymptotic expansion of $\left(\overline{\partial_{00}^{2} g_{A B}}\right)$, solution to $\left(\overline{\partial_{0} R_{A B}}\right)=0$. However, this ODE is of exactly the same form as the ODE (4.42) for $\overline{\partial_{0} g_{A B}}$. Therefore an identical argument applies and leads to the conclusion that the solution can be asymptotically expanded as a power series,

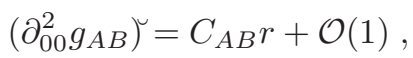

where the symmetric and $s$-trace-free tensor $C_{A B}$ can be identified with the integration functions.

For completeness let us also compute

$$
\begin{aligned}
\overline{\partial_{0} \Gamma_{0 A}^{r}} & =\frac{1}{2} \overline{\partial_{00}^{2} g_{r A}}-\frac{1}{2} \xi^{B} \overline{\partial_{0} g_{A B}}+\frac{r}{2}|\sigma|^{2} \xi_{A} \\
& =-\frac{1}{2} \stackrel{\nabla}{\nabla}_{B} D_{A}{ }^{B} r^{-1}+\mathcal{O}\left(r^{-2}\right) .
\end{aligned}
$$




\subsection{Overview of the metric gauge II}

We give an overview of the values of all those objects we have computed so far in the metric gauge. Recall the values for the gauge functions $\kappa, \bar{W}^{\lambda}$ and $\overline{\partial_{0} W^{\lambda}}$ as given by (4.29)-(4.32), (4.49), 4.56) and (4.63), needed to realize these values. Recall further that equality is meant to hold for $r>r_{2}$.

\section{Metric components}

$$
\begin{array}{ll}
\bar{g}_{00}=-1, & \bar{g}^{00}=0, \\
\bar{g}_{0 r}=1, & \bar{g}^{0 r}=1, \\
\bar{g}_{0 A}=0, & \bar{g}^{0 A}=0, \\
\bar{g}_{r r}=0, & \bar{g}^{r r}=1, \\
\bar{g}_{r A}=0, & \bar{g}^{r A}=0, \\
\bar{g}_{A B}=r^{2} \sqrt{\frac{\operatorname{det} s}{\operatorname{det} \gamma}} \gamma_{A B}=r^{2} s_{A B}+\mathcal{O}(r), & \bar{g}^{A B}=r^{-2}
\end{array}
$$

First-order $u$-derivatives of the metric components

$$
\begin{aligned}
& \overline{\partial_{0} g_{00}}=0 \text {, } \\
& \overline{\partial_{0} g_{0 r}}=0 \text {, } \\
& \overline{\partial_{0} g_{0 A}}=0 \text {, } \\
& \overline{\partial_{0} g^{00}}=r|\sigma|^{2}=\mathcal{O}\left(r^{-3}\right), \\
& \overline{\partial_{0} g_{r r}}=-r|\sigma|^{2}=\mathcal{O}\left(r^{-3}\right) \text {, } \\
& \overline{\partial_{0} g_{r A}}=\xi_{A}=\mathcal{O}\left(r^{-1}\right) \text {, } \\
& \overline{\partial_{0} g_{A B}}=D_{A B} r+\mathcal{O}(1) \text {, } \\
& \overline{\partial_{0} g^{0 r}}=r|\sigma|^{2}=\mathcal{O}\left(r^{-3}\right), \\
& \overline{\partial_{0} g^{0 A}}=-\xi^{A}=\mathcal{O}\left(r^{-3}\right), \\
& \overline{\partial_{0} g^{r r}}=r|\sigma|^{2}=\mathcal{O}\left(r^{-3}\right), \\
& \overline{\partial_{0} g^{r A}}=-\xi^{A}=\mathcal{O}\left(r^{-3}\right) \text {, } \\
& \overline{\partial_{0} g^{A B}}=-D^{A B} r^{-3}+\mathcal{O}\left(r^{-4}\right) \text {. }
\end{aligned}
$$

Second-order $u$-derivatives of the metric components

$$
\begin{aligned}
\overline{\partial_{00}^{2} g_{r r}} & =\Xi r^{-3}+\mathcal{O}\left(r^{-4}\right), \\
\overline{\partial_{00}^{2} g_{r A}} & =-{ }^{\circ}{ }_{B} D_{A}^{B} r^{-1}+\mathcal{O}\left(r^{-2}\right), \\
\bar{g}^{A B} \overline{\partial_{00}^{2} g_{A B}} & =\frac{1}{2}|D|^{2} r^{-2}+\mathcal{O}\left(r^{-3}\right) .
\end{aligned}
$$

Asymptotic behavior of the Christoffel symbols (cf. p. 27)

$$
\begin{aligned}
& \bar{\Gamma}_{00}^{0}=0 \text {, } \\
& \bar{\Gamma}_{r r}^{r}=\mathcal{O}\left(r^{-3}\right) \text {, } \\
& \bar{\Gamma}_{0 r}^{0}=\mathcal{O}\left(r^{-3}\right) \text {, } \\
& \bar{\Gamma}_{r A}^{r}=\frac{1}{2} \stackrel{\circ}{\nabla}_{B} h_{A}^{(1) B} r^{-1}+\mathcal{O}\left(r^{-2}\right), \\
& \bar{\Gamma}_{0 A}^{0}=-\frac{1}{2} \stackrel{\circ}{\nabla}_{B} \breve{h}_{A}^{(1)} B_{B} r^{-1}+\mathcal{O}\left(r^{-2}\right), \quad \bar{\Gamma}_{A B}^{r}=-\left(s_{A B}+\frac{1}{2} D_{A B}\right) r+\mathcal{O}(1), \\
& \bar{\Gamma}_{r r}^{0}=0, \quad \bar{\Gamma}_{00}^{C}=0, \\
& \bar{\Gamma}_{r A}^{0}=0 \text {, } \\
& \bar{\Gamma}_{A B}^{r A}=-r s_{A B}+\mathcal{O}(1) \text {, } \\
& \bar{\Gamma}_{00}^{r}=0 \text {, } \\
& \bar{\Gamma}_{0 r}^{C}=-\frac{1}{2} \stackrel{\circ}{\nabla}^{B} h_{B}^{(1) C} r^{-3}+\mathcal{O}\left(r^{-4}\right), \\
& \bar{\Gamma}_{0 r}^{r}=\mathcal{O}\left(r^{-3}\right) \text {, } \\
& \bar{\Gamma}_{0 A}^{C}=\frac{1}{2} D_{A}{ }^{C} r^{-1}+\mathcal{O}\left(r^{-2}\right) \text {, } \\
& \bar{\Gamma}_{r r}^{C A}=0, \\
& \bar{\Gamma}_{0 A}^{r}=-\frac{1}{2} \stackrel{\circ}{\nabla}_{B} \breve{h}_{A}^{(1) B} r^{-1}+\mathcal{O}\left(r^{-2}\right), \\
& \bar{\Gamma}_{r A}^{C r}=\frac{1}{r} \delta_{A}^{C}+\mathcal{O}\left(r^{-2}\right) \text {, }
\end{aligned}
$$

First-order $u$-derivatives of the Christoffel symbols

We give a list of the asymptotic behavior of all the $u$-differentiated Christoffel symbols crucial e.g. for the computation of the Weyl tensor, which can be straightforwardly derived from our previous results, 


$$
\begin{aligned}
& \overline{\partial_{0} \Gamma_{00}^{0}}=\text { not needed, } \quad \overline{\partial_{0} \Gamma_{r r}^{r}}=-\frac{1}{2} \Xi r^{-3}+\mathcal{O}\left(r^{-4}\right) \text {, } \\
& \overline{\partial_{0} \Gamma_{0 r}^{0}}=\frac{1}{2} \Xi r^{-3}+\mathcal{O}\left(r^{-4}\right), \quad \overline{\partial_{0} \Gamma_{r A}^{r}}=\frac{1}{2} \nabla_{B} D_{A}{ }^{B} r^{-1}+\mathcal{O}\left(r^{-2}\right), \\
& \overline{\partial_{0} \Gamma_{0 A}^{0}}=-\frac{1}{2} \stackrel{\circ}{\nabla}_{B} D_{A}{ }^{B} r^{-1}+\mathcal{O}\left(r^{-2}\right), \quad \overline{\partial_{0} \Gamma_{A B}^{r}}=-\frac{1}{2} C_{A B} r+\mathcal{O}(1), \\
& \overline{\partial_{0} \Gamma_{r r}^{0}}=\frac{3}{8}\left|h^{(1)}\right|^{2} r^{-4}+\mathcal{O}\left(r^{-5}\right), \quad \overline{\partial_{0} \Gamma_{00}^{C}}=\text { not needed, } \\
& \overline{\partial_{0} \Gamma_{r A}^{0}}=\stackrel{\circ}{\nabla}_{B} h_{A}^{(1) B} r^{-2}+\mathcal{O}\left(r^{-3}\right), \quad \frac{\overline{\partial_{0} \Gamma_{0 r}^{C}}}{\partial_{0} \Gamma_{A B}}=-\frac{1}{2} \stackrel{\circ}{\nabla}_{B} D^{B C} r^{-3}+\mathcal{O}\left(r^{-4}\right), \\
& \overline{\partial_{0} \Gamma_{A B}^{0}}=-\frac{1}{2} D_{A B}+\mathcal{O}\left(r^{-1}\right), \quad \overline{\partial_{0} \Gamma_{0 A}^{C}}=\frac{1}{2} C_{A}^{C} r^{-1}+\mathcal{O}\left(r^{-2}\right), \\
& \overline{\partial_{0} \Gamma_{00}^{r}}=\text { not needed, } \quad \overline{\partial_{0} \Gamma_{r r}^{C}}=\nabla^{B} h_{B}^{(1) C} r^{-4}+\mathcal{O}\left(r^{-5}\right) \text {, } \\
& \overline{\partial_{0} \Gamma_{0 r}^{r}}=\frac{1}{2} \Xi r^{-3}+\mathcal{O}\left(r^{-4}\right), \quad \overline{\partial_{0} \Gamma_{r A}^{C}}=-\frac{1}{2} D_{A}{ }^{C} r^{-2}+\mathcal{O}\left(r^{-3}\right) \text {, } \\
& \overline{\partial_{0} \Gamma_{0 A}^{r}}=-\frac{1}{2} \stackrel{\circ}{\nabla}_{B} D_{A}{ }^{B} r^{-1}+\mathcal{O}\left(r^{-2}\right), \quad \overline{\partial_{0} \Gamma_{A B}^{C}}=\left[\stackrel{\circ}{\nabla}_{(A} D_{B)}{ }^{C}-\frac{1}{2} \stackrel{\circ}{ }^{C} D_{A B}\right] r^{-1}+\mathcal{O}\left(r^{-2}\right) \text {. }
\end{aligned}
$$

\section{Asymptotic Expansions of the unknowns of the CFE on $\mathscr{N}$}

\subsection{Conformal field equations (CFE)}

As indicated in the introduction, one would like to establish an existence theorem for the characteristic initial value problem for the vacuum Einstein equations which guarantees a "piece of a smooth $\mathscr{I}^{+}$". This global existence problem, where one needs to control the limiting behavior near null infinity, can be transformed into a local one via a conformal rescaling $g \mapsto \tilde{g}=\Theta^{2} g$, where the conformal factor $\Theta$ has to vanish on the hypersurface $\mathscr{I}^{+}$which represents (future) null infinity (with $\mathrm{d} \Theta \neq 0$ there). Henceforth we use ${ }^{\sim}$ to label objects related to the unphysical space-time metric $\tilde{g}$.

The Einstein equations, regarded as equations for $\tilde{g}$ and $\Theta$, become singular wherever $\Theta$ vanishes, and are therefore not suitable to tackle the issue of proving existence of a solution near $\mathscr{I}^{+}$. However, H. Friedrich was able to find a representation of the vacuum Einstein equations which remains regular even where the conformal factor vanishes, cf. e.g. 8]. These conformal field equations $(C F E)$ treat $\Theta$ as an unknown rather than a gauge function. Its gauge freedom is reflected in the freedom to prescribe the curvature scalar $\tilde{R}$. This still leaves the freedom to prescribe $\Theta$ on the initial surface. We shall take

$$
\bar{\Theta}=1 / r=x \quad \text { for } r>r_{2}
$$

as initial data on $\mathscr{N}$. The CFE give rise to a complicated and highly overdetermined system of PDEs, which, in 4 space-time dimensions, can be represented as a symmetric hyperbolic system, supplemented by certain constraint equations.

\subsubsection{Metric conformal field equations (MCFE)}

There exist different versions of the CFE depending on which fields are regarded as unknowns. Let us first introduce the metric conformal field equations (MCFE) [8]. Beside $\tilde{g}$ and $\Theta$ the unknowns are the Schouten tensor,

$$
\tilde{L}_{\mu \nu}=\frac{1}{2} \tilde{R}_{\mu \nu}-\frac{1}{12} \tilde{R} \tilde{g}_{\mu \nu},
$$

the rescaled Weyl tensor,

$$
\tilde{d}_{\mu \nu \sigma}{ }^{\rho}=\Theta^{-1} \tilde{C}_{\mu \nu \sigma}{ }^{\rho}=\Theta^{-1} C_{\mu \nu \sigma}{ }^{\rho},
$$


and the scalar function (set $\square_{\tilde{g}}=\tilde{g}^{\mu \nu} \tilde{\nabla}_{\mu} \tilde{\nabla}_{\nu}$ )

$$
\tilde{s}=\frac{1}{4} \square_{\tilde{g}} \Theta+\frac{1}{24} \tilde{R} \Theta .
$$

The MCFE read

$$
\begin{aligned}
& \tilde{R}_{\mu \nu \sigma}{ }^{\kappa}[\tilde{g}]=\Theta \tilde{d}_{\mu \nu \sigma}{ }^{\kappa}+2\left(\tilde{g}_{\sigma[\mu} \tilde{L}_{\nu]}{ }^{\kappa}-\delta_{[\mu}{ }^{\kappa} \tilde{L}_{\nu] \sigma}\right) \\
& \tilde{\nabla}_{\rho} \tilde{d}_{\mu \nu \sigma}{ }^{\rho}=0, \\
& \tilde{\nabla}_{\mu} \tilde{L}_{\nu \sigma}-\tilde{\nabla}_{\nu} \tilde{L}_{\mu \sigma}=\tilde{\nabla}_{\rho} \Theta \tilde{d}_{\nu \mu \sigma}{ }^{\rho}, \\
& \tilde{\nabla}_{\mu} \tilde{\nabla}_{\nu} \Theta=-\Theta \tilde{L}_{\mu \nu}+\tilde{s} \tilde{g}_{\mu \nu}, \\
& \tilde{\nabla}_{\mu} \tilde{s}=-\tilde{L}_{\mu \nu} \tilde{\nabla}^{\nu} \Theta, \\
& 2 \Theta \tilde{s}-\tilde{\nabla}_{\mu} \Theta \tilde{\nabla}^{\mu} \Theta=0, .
\end{aligned}
$$

Equation (5.7) is a consequence of (5.5) and (5.6) if it is known to hold at one point (e.g. by an appropriate choice of the initial data).

\subsubsection{General conformal field equations (GCFE)}

Consider any frame field $e_{k}=e^{\mu}{ }_{k} \partial_{\mu}$ such that the $\tilde{g}\left(e_{i}, e_{k}\right) \equiv \tilde{g}_{i k}$ 's are constants. The general conformal field equations (GCFE) [8] for the unknowns

$$
\begin{array}{llllll}
e^{\mu}{ }_{k}, & \tilde{\Gamma}_{i}{ }^{k}{ }_{j}, & \tilde{d}_{i j k}{ }^{l}, & \tilde{L}_{i j}, & \Theta, & \tilde{s}
\end{array}
$$

read (from now on Latin indices are used to denote frame-components)

$$
\begin{aligned}
& {\left[e_{p}, e_{q}\right]=\left(\tilde{\Gamma}_{p}^{l}{ }^{l}-\tilde{\Gamma}_{q p}^{l}\right) e_{l}} \\
& \left.e_{[p}\left(\tilde{\Gamma}_{q]}{ }^{i} j\right)-\tilde{\Gamma}_{k}{ }_{j}{ }_{j} \tilde{\Gamma}_{[p}{ }^{k} q\right]+\tilde{\Gamma}_{[p|k|}{ }^{i} \mid \tilde{\Gamma}_{q]}{ }^{k}{ }_{j}=\delta_{[p}{ }^{i} \tilde{L}_{q] j}-\tilde{g}_{j[p} \tilde{L}_{q]}{ }^{i}-\frac{1}{2} \Theta \tilde{d}_{p q j}{ }^{i} \\
& \tilde{\nabla}_{i} \tilde{d}_{p q j}{ }^{i}=0 \\
& \tilde{\nabla}_{i} \tilde{L}_{j k}-\tilde{\nabla}_{j} \tilde{L}_{i k}=\tilde{\nabla}_{l} \Theta \tilde{d}_{j i k}{ }^{l} \\
& \tilde{\nabla}_{i} \tilde{\nabla}_{j} \Theta=-\Theta \tilde{L}_{i j}+s \tilde{g}_{i j} \\
& \tilde{\nabla}_{i} \tilde{s}=-\tilde{L}_{i j} \tilde{\nabla}^{j} \Theta \\
& 2 \Theta \tilde{s}-\tilde{\nabla}_{j} \Theta \tilde{\nabla}^{j} \Theta=0
\end{aligned}
$$

where the $\tilde{\Gamma}_{i}{ }^{j}{ }_{k}$ 's denote the Levi-Civita connection coefficients in the frame $e_{k}$. Again, (5.14) suffices to be satisfied at just one point.

\subsection{Asymptotic behavior of the fields appearing in the CFE}

In this section we analyze the asymptotic behavior of the restriction to $\mathscr{N}$ of the fields $\tilde{g}_{\mu \nu}, e_{k}^{\mu}, \tilde{\Gamma}_{\mu \nu}^{\sigma}, \tilde{d}_{\mu \nu \sigma}{ }^{\rho}, \tilde{L}_{\mu \nu}, \Theta$ and $\tilde{s}$ and prove that they are smooth at infinity in the metric gauge and taking (5.1) when constructed as solutions of the constraint equations induced by the wave-map gauge reduced vacuum Einstein equations, supposing that the initial data $\gamma$ are of the form (2.15), and the no-logs-condition (3.49) hold. As we shall show that the above fields are smooth at $\mathscr{I}^{+}$w.r.t the $e_{k}$-frame if and only if they have this property in the coordinate frame defined by $\left\{u, x, x^{A}\right\}$, we shall end up with the result that (2.15) and (3.49) lead to smooth initial data for both MCFE and GCFE.

Using our previous results summarized in Section 4.7 most of the computations will be straightforward. 


\subsubsection{Asymptotic behavior of the metric tensor}

It follows from (4.33)- (4.34) that 6

$$
\overline{\tilde{g}}_{00}=-x^{2}, \quad \overline{\tilde{g}}_{0 x}=-1, \quad \overline{\tilde{g}}_{0 A}=\overline{\tilde{g}}_{x x}=\overline{\tilde{g}}_{x A}=0, \quad \overline{\tilde{g}}_{A B}=s_{A B}+\mathcal{O}(x),
$$

i.e. $\overline{\tilde{g}}$ has a smooth conformal completion at conformal infinity.

\subsubsection{Asymptotic behavior of the Weyl tensor}

Note that the rescaled Weyl tensor $\overline{\tilde{d}}_{\mu \nu \sigma^{\rho}}{ }^{\rho}=\mathcal{O}(1)$ will be smooth at $\mathscr{I}^{+}$if and only if the Weyl tensor is smooth at $\mathscr{I}^{+}$and vanishes there, i.e. $\tilde{\widetilde{C}}_{\mu \nu \sigma}{ }^{\rho}=$ $\bar{C}_{\mu \nu \sigma}{ }^{\rho}=\mathcal{O}(x)$.

In vacuum we have $R_{\mu \nu}=0$, and the Weyl tensor coincides with the Riemann tensor,

$$
\bar{C}_{\mu \nu \rho}^{\sigma}=\bar{R}_{\mu \nu \rho}^{\sigma}=\overline{\partial_{\nu} \Gamma_{\mu \rho}^{\sigma}}-\overline{\partial_{\mu} \Gamma_{\nu \rho}^{\sigma}}+\bar{\Gamma}_{\mu \rho}^{\alpha} \bar{\Gamma}_{\alpha \nu}^{\sigma}-\bar{\Gamma}_{\nu \rho}^{\alpha} \bar{\Gamma}_{\alpha \mu}^{\sigma} .
$$

Due to its algebraic symmetries it suffices to consider the components

$$
\begin{aligned}
& \bar{C}_{0 r 0}{ }^{0}=\mathcal{O}\left(r^{-3}\right), \quad \bar{C}_{0 r A^{0}}=\mathcal{O}\left(r^{-3}\right), \quad \bar{C}_{0 A 0^{0}}=\mathcal{O}\left(r^{-1}\right), \\
& \bar{C}_{0 A 0}{ }^{B}=\mathcal{O}\left(r^{-1}\right), \quad \bar{C}_{A B 0^{0}}=\mathcal{O}\left(r^{-1}\right), \quad \bar{C}_{r A B}{ }^{0}=\mathcal{O}\left(r^{-3}\right) \text {, }
\end{aligned}
$$

as follows from the formulae in Section 4.7. Remarkably all the leading order terms which would induce terms of constant order after carrying out the coordinate transformation $r \mapsto x:=1 / r$ cancel out, in particular those involving some of the integration constants whose explicit values are not known,

$$
\begin{aligned}
\bar{C}_{0 x 0^{0}}=\mathcal{O}(x), & \bar{C}_{0 x A}{ }^{0}=\mathcal{O}(x), & \bar{C}_{0 A 0^{0}}=\mathcal{O}(x), \\
\bar{C}_{0 A 0}{ }^{B}=\mathcal{O}(x), & \bar{C}_{A B 0}{ }^{0}=\mathcal{O}(x), & \bar{C}_{x A B}{ }^{0}=\mathcal{O}(x) .
\end{aligned}
$$

(Recall that $\mathcal{O}(x)$ has been defined in Section 2.1) To establish that $\bar{C}_{r A B}{ }^{0}=$ $\mathcal{O}\left(r^{-3}\right)$ rather than $\bar{C}_{r A B}{ }^{0}=\mathcal{O}\left(r^{-2}\right)$ one needs to employ the no-logs-condition and is led to the geometric interpretation described in [6, Section 6.2]. No further condition on the initial data needs to be imposed.

\subsubsection{Asymptotic behavior of $\overline{\partial_{0} \Theta}$ and $\overline{\partial_{00}^{2} \Theta}$}

To compute the remaining fields on $\mathscr{N}$ we first need to determine the trace of the first- and second-order $u$-derivative of the conformal factor $\Theta$ on $\mathscr{N}$. However, the values of $\Theta$ away from $\mathscr{N}$ depend on the unphysical curvature scalar $\tilde{R}$, which is treated as a conformal gauge source function in the CFE 8]. We impose the gauge condition

$$
\overline{\tilde{R}}=\mathcal{O}(1), \quad \overline{\tilde{\nabla}_{0} \tilde{R}}=\mathcal{O}(1)
$$

(which is no restriction since $\tilde{R}$ needs to be smooth at $\mathscr{I}^{+}$anyway). The Ricci scalars of $\tilde{g}=\Theta^{2} g$ and $g$ are related via

$$
\tilde{R}=\Theta^{-2}\left(R-6 \Theta^{-1} g^{\rho \sigma} \partial_{\rho} \partial_{\sigma} \Theta+6 \Theta^{-1} g^{\rho \sigma} \Gamma_{\rho \sigma}^{\alpha} \partial_{\alpha} \Theta\right) .
$$

${ }^{6}$ While up to now we used the variable $x=1 / r$ mainly as an auxiliary quantity to facilitate the comparison of the constraint equations with the formulae in Appendix $\mathrm{A}$ we shall use it henceforth as a coordinate. 
With $R=0$ that yields an ODE for $\overline{\partial_{0} \Theta}$ on $\mathscr{N}$ where $\bar{\Theta}=1 / r$,

$$
\begin{aligned}
\overline{\tilde{R}} & =6 r^{2}\left[-2 r\left(\partial_{r}+r^{-1}+\frac{r}{2}|\sigma|^{2}\right) \overline{\partial_{0} \Theta}+|\sigma|^{2}+\frac{1}{2} r^{-1} \bar{g}^{A B} \overline{\partial_{0} g_{A B}}\right] \\
& =-12 r^{3}\left(\partial_{r}+r^{-1}+\mathcal{O}\left(r^{-3}\right)\right) \overline{\partial_{0} \Theta}+\mathcal{O}\left(r^{-1}\right) .
\end{aligned}
$$

Employing (5.15) it takes the form

$$
r \partial_{r} \overline{\partial_{0} \Theta}+\left[1+\mathcal{O}\left(r^{-2}\right)\right] \overline{\partial_{0} \Theta}=\mathcal{O}\left(r^{-2}\right) .
$$

Appendix $\$$ tells us (with $\lambda=\hat{\ell}=1$ ) that the asymptotic expansion does not contain logarithmic terms and is of the form

$$
\overline{\partial_{0} \Theta}=\mathcal{O}\left(r^{-1}\right)=\mathcal{O}(x) .
$$

The second-order $u$-derivative of the conformal factor can be computed as follows: From (5.16) we deduce with $\overline{\partial_{0} R}=0$ that

$$
\begin{aligned}
\overline{\partial_{0} \tilde{R}} & =6 \overline{\partial_{0}\left(\Theta^{-3} g^{\rho \sigma} \Gamma_{\rho \sigma}^{\alpha} \partial_{\alpha} \Theta-\Theta^{-3} g^{\rho \sigma} \partial_{\rho} \partial_{\sigma} \Theta\right)} \\
& =-3 r \tilde{\tilde{R}} \overline{\partial_{0} \Theta}-12 r^{3}\left(\partial_{r}+r^{-1}+\mathcal{O}\left(r^{-3}\right)\right) \overline{\partial_{00}^{2} \Theta}+\mathcal{O}(1) .
\end{aligned}
$$

Taking the gauge condition (5.15) into account this ODE for $\overline{\partial_{00}^{2} \Theta}$ becomes

$$
r \partial_{r} \overline{\partial_{00}^{2} \Theta}+\left[1+\mathcal{O}\left(r^{-2}\right)\right] \overline{\partial_{00}^{2} \Theta}=\mathcal{O}\left(r^{-2}\right),
$$

which is of the same form as (5.19). Hence

$$
\overline{\partial_{00}^{2} \Theta}=\mathcal{O}\left(r^{-1}\right)=\mathcal{O}(x) .
$$

\subsubsection{Asymptotic behavior of the Christoffel symbols}

We have computed the restriction to $\mathscr{N}$ of the Christoffel symbols in adapted null coordinates $\left(u, r, x^{A}\right)$ by imposing the metric gauge condition, cf. Section 4.7. Using the well-known behavior of Christoffel symbols under coordinate transformations we determine their asymptotic behavior in the $\left(u, x=1 / r, x^{A}\right)$ coordinates,

$$
\begin{array}{ll}
\bar{\Gamma}_{00}^{0}=0, & \bar{\Gamma}_{x x}^{x}=-2 x^{-1}+\mathcal{O}(x), \\
\bar{\Gamma}_{0 x}^{0}=\mathcal{O}(x), & \bar{\Gamma}_{x A}^{x}=\mathcal{O}(x), \\
\bar{\Gamma}_{0 A}^{0}=\mathcal{O}(x), & \bar{\Gamma}_{A B}^{x}=\left(s_{A B}+\frac{1}{2} D_{A B}\right) x+\mathcal{O}\left(x^{2}\right), \\
\bar{\Gamma}_{x x}^{0}=0, & \bar{\Gamma}_{00}^{C}=0 \\
\bar{\Gamma}_{x A}^{0}=0, & \bar{\Gamma}_{0 x}^{C}=\mathcal{O}(x) \\
\bar{\Gamma}_{A B}^{0}=-x^{-1} s_{A B}+\mathcal{O}(1), & \bar{\Gamma}_{0 A}^{C}=\frac{1}{2} D_{A}{ }^{C} x+\mathcal{O}\left(x^{2}\right), \\
\bar{\Gamma}_{00}^{x}=0, & \bar{\Gamma}_{x x}^{C}=0 \\
\bar{\Gamma}_{0 x}^{x}=\mathcal{O}\left(x^{3}\right), & \bar{\Gamma}_{x A}^{C}=-\delta_{A}^{C} x^{-1}+\mathcal{O}(1), \\
\bar{\Gamma}_{0 A}^{x}=\mathcal{O}\left(x^{3}\right), & \bar{\Gamma}_{A B}^{C}=\Gamma_{A B}^{C}+\mathcal{O}(x) .
\end{array}
$$

We compute the trace of the Christoffel symbols on $\mathscr{N}$ associated to the unphysical metric $\tilde{g}$. The transformation law for Christoffel symbols under a conformal rescaling $g \mapsto \Theta^{2} g$ of the metric reads,

$$
\tilde{\Gamma}_{\mu \nu}^{\rho}=\Gamma_{\mu \nu}^{\rho}+\frac{1}{\Theta}\left(\delta_{\nu}{ }^{\rho} \partial_{\mu} \Theta+\delta_{\mu}{ }^{\rho} \partial_{\nu} \Theta-g_{\mu \nu} g^{\rho \sigma} \partial_{\sigma} \Theta\right),
$$


which yields on $\mathscr{N}$, where $\bar{\Theta}=x$ and $\overline{\partial_{0} \Theta}=\mathcal{O}(x)$,

$$
\begin{gathered}
\overline{\tilde{\Gamma}}_{00}^{0}=\mathcal{O}(1), \quad \overline{\tilde{\Gamma}}_{0 x}^{0}=\mathcal{O}(x), \quad \overline{\tilde{\Gamma}}_{0 A}^{0}=\mathcal{O}(x), \quad \overline{\tilde{\Gamma}}_{x x}^{0}=0, \quad \overline{\tilde{\Gamma}}_{x A}^{0}=0, \\
\overline{\tilde{\Gamma}}_{A B}^{0}=\mathcal{O}(1), \quad \overline{\tilde{\Gamma}}_{00}^{x}=\mathcal{O}\left(x^{2}\right), \quad \overline{\tilde{\Gamma}}_{0 x}^{x}=\mathcal{O}(x), \quad \overline{\tilde{\Gamma}}_{0 A}^{x}=\mathcal{O}\left(x^{3}\right), \\
\overline{\tilde{\Gamma}}_{x x}^{x}=\mathcal{O}(1), \quad \overline{\tilde{\Gamma}}_{x A}^{x}=\mathcal{O}(x), \quad \overline{\tilde{\Gamma}}_{A B}^{x}=\mathcal{O}(1), \quad \overline{\tilde{\Gamma}}_{00}^{C}=0, \quad \overline{\tilde{\Gamma}}_{0 x}^{C}=\mathcal{O}(x), \\
\overline{\tilde{\Gamma}}_{0 A}^{C}=\mathcal{O}(1), \quad \overline{\tilde{\Gamma}}_{x x}^{C}=0, \quad \overline{\tilde{\Gamma}}_{x A}^{C}=\mathcal{O}(1), \quad \bar{\Gamma}_{A B}^{C}=\mathcal{O}(1) .
\end{gathered}
$$

The Christoffel symbols are smooth without any further restrictions on $\gamma$.

\subsubsection{Asymptotic behavior of the Schouten tensor}

From now all tensors will be expressed in terms of the coordinates $\left(u, x, x^{A}\right)$. We compute the Schouten tensor $\tilde{L}_{\mu \nu}=\frac{1}{2} \tilde{R}_{\mu \nu}-\frac{1}{12} \tilde{R} \tilde{g}_{\mu \nu}$, restricted to $\mathscr{N}$, for the conformally rescaled metric $\tilde{g}=\Theta^{2} g$. The transformation law for the Ricci tensor under conformal rescalings of the metric reads,

$$
\tilde{L}_{\mu \nu}=L_{\mu \nu}-\Theta^{-1}\left(\partial_{\mu} \partial_{\nu} \Theta-\Gamma_{\mu \nu}^{\alpha} \partial_{\alpha} \Theta\right)+2 \Theta^{-2}\left(\partial_{\mu} \Theta \partial_{\nu} \Theta\right)^{\nu} .
$$

With $\bar{L}_{\mu \nu}=0$ we obtain on $\mathscr{N}$

$\overline{\tilde{L}}_{\mu \nu}=2 x^{-2} \overline{\partial_{\mu} \Theta} \overline{\partial_{\nu} \Theta}-x^{-1} \overline{\partial_{\mu} \partial_{\nu} \Theta}+\left(x^{-1} \bar{\Gamma}_{\mu \nu}^{0}+\bar{g}_{\mu \nu}\right) \overline{\partial_{0} \Theta}+x^{-1} \bar{\Gamma}_{\mu \nu}^{x}-\frac{1}{2} x^{2} \bar{g}_{\mu \nu}$.

Assuming (5.15), so that (5.19) and (5.21) hold, we find

$$
\begin{aligned}
\overline{\tilde{L}}_{00} & =2 x^{-2} \overline{\partial_{0} \Theta} \overline{\partial_{0} \Theta}-\overline{\partial_{0} \Theta}+\frac{1}{2} x^{2}-x^{-1} \overline{\partial_{00}^{2} \Theta}=\mathcal{O}(1) \\
\overline{\tilde{L}}_{0 x} & =\frac{1}{2}-\partial_{x}\left(x^{-1} \overline{\partial_{0} \Theta}\right)+\mathcal{O}(1) \overline{\partial_{0} \Theta}+\mathcal{O}\left(x^{2}\right)=\mathcal{O}(1) \\
\overline{\tilde{L}}_{0 A} & =-x^{-1} \partial_{A} \overline{\partial_{0} \Theta}+\mathcal{O}(1) \overline{\partial_{0} \Theta}+\mathcal{O}\left(x^{2}\right)=\mathcal{O}(1) \\
\overline{\tilde{L}}_{x x} & =\mathcal{O}(1) \\
\overline{\tilde{L}}_{x A} & =\mathcal{O}(1) \\
\bar{L}_{A B} & =\mathcal{O}\left(x^{-1}\right) \overline{\partial_{0} \Theta}+\mathcal{O}(1)=\mathcal{O}(1)
\end{aligned}
$$

We conclude that the trace of the Schouten tensor on $\mathscr{N}$ is smooth at conformal infinity.

\subsubsection{Asymptotic behavior of the function $\tilde{s}$}

Let us determine the asymptotic behavior of the function $\tilde{s} \equiv \frac{1}{4} \square_{\tilde{g}} \Theta+\frac{1}{24} \tilde{R} \Theta$ on $\mathscr{N}$. Using (5.16) with $\bar{R}=0$ and (5.22) we find that

$$
\begin{aligned}
\overline{\tilde{s}} & =\frac{1}{4} x^{-2} \bar{g}^{\mu \nu} \partial_{\mu} \partial_{\nu} \Theta-\frac{1}{4} x^{-2} \bar{g}^{\mu \nu} \tilde{\Gamma}_{\mu \nu}^{\kappa} \overline{\partial_{\kappa} \Theta}+\frac{1}{24} \overline{\tilde{R}} x \\
& =\frac{1}{24} x^{-1} \bar{R}+\frac{1}{4} x^{-2} \bar{g}^{\mu \nu}\left(\bar{\Gamma}_{\mu \nu}^{\alpha}-\overline{\tilde{\Gamma}}_{\mu \nu}^{\alpha}\right) \partial_{\alpha} \Theta \\
& =\frac{1}{2} x^{-3} \bar{g}^{\alpha \beta} \overline{\partial_{\alpha} \Theta} \overline{\partial_{\beta} \Theta},
\end{aligned}
$$


which recovers (5.6), and which yields with (5.19) that

$$
\overline{\tilde{s}}=\frac{1}{2} x-x^{-1} \overline{\partial_{0} \Theta}=\mathcal{O}(1),
$$

i.e. the function $\overline{\tilde{s}}$ is smooth at conformal infinity.

\section{Asymptotic behavior of the frame field}

The GCFE (5.8)-(5.14) require a frame field $\left(e^{\mu}{ }_{k}\right)$ w.r.t which the metric tensor is constant. In our coordinates the trace of $\tilde{g}$ on $\mathscr{N}$ looks as follows (recall that equality is meant to hold for $r>r_{2}$ ):

$$
\overline{\tilde{g}}=-x^{2} \mathrm{~d} u^{2}-2 \mathrm{~d} u \mathrm{~d} x+\tilde{g}_{A B} \mathrm{~d} x^{A} \mathrm{~d} x^{B} \quad \text { with } \quad \tilde{g}_{A B}=x^{2} \bar{g}_{A B}=\mathcal{O}(1) .
$$

We deduce that we may take $\left(e^{\mu}{ }_{k}\right)$ to be of the following form on $\mathscr{N}$ :

$$
\begin{aligned}
\bar{e}_{0} & =\partial_{u}-\frac{x^{2}}{4} \partial_{x}, \\
\bar{e}_{x} & =\partial_{x}, \\
\bar{e}_{\tilde{A}} & =e^{A}{ }_{\tilde{A}} \partial_{A} \quad \text { with } e_{\tilde{A}}^{A}=\mathcal{O}(1) \quad \tilde{A}=2,3,
\end{aligned}
$$

and its dual

$$
\begin{aligned}
\bar{\Theta}^{0} & =\mathrm{d} u, \\
\bar{\Theta}^{x} & =\mathrm{d} x+\frac{x^{2}}{4} \mathrm{~d} u, \\
\bar{\Theta}^{\tilde{A}} & =\hat{e}^{\tilde{A}}{ }_{A} \mathrm{~d} x^{A} \quad \text { with } \quad \hat{e}_{A}^{\tilde{A}}=\mathcal{O}(1) \quad \tilde{A}=2,3 .
\end{aligned}
$$

All the relevant fields are smooth at $\mathscr{I}^{+}$w.r.t. this frame if and only if they have this property w.r.t. the coordinate frame defined by the adapted coordinates $\left\{u, x, x^{A}\right\}$, which we have shown to be the case.

\subsection{Main result}

Consider a space-time $(\mathscr{M}, g)$ which admits a smooth conformal completion at infinity à la Penrose, and consider a null hypersurface $\mathscr{N} \subset \mathscr{M}$ whose closure in the conformally completed space-time $\mathscr{M} \cup \mathscr{I}^{+}$is smooth and meets $\mathscr{I}^{+}$in a smooth spherical cross-section. It follows from the considerations in 11], cf. [7], that then one can introduce Bondi coordinates near $\overline{\mathscr{N}} \cap \mathscr{I}^{+}$w.r.t. which $\mathscr{N}$ intersects $\mathscr{I}^{+}$at the surface $\{u=0\}$, and in which all the fields appearing in the CFE are smooth at $\mathscr{I}^{+}$in the sense of Definition 2.1.

The existence of adapted null coordinates in which the unknowns of the CFE are smooth at $\mathscr{I}^{+}$is thus a necessary condition for the existence of a space-time which admits a smooth conformal completion à la Penrose. We are led to the following result:

THEOREM 5.1 A necessary-and-sufficient condition for the restrictions to $\mathscr{N}$ of all the fields appearing in the GCFE (5.8)-(5.14), or the MCFE (5.2)-(5.7), constructed from initial data $\gamma=\gamma_{A B} \mathrm{~d} x^{A} \mathrm{~d} x^{B}$ of the form (2.15) to be smooth at $\mathscr{I}^{+}$, is that the initial data $\gamma$ satisfy the no-logs-condition 3.49) in some (and then all) adapted null coordinate systems. In that case the metric gauge provides a gauge choice where smoothness at $\mathscr{I}^{+}$holds. 
Acknowledgments I am grateful to my advisor Piotr T. Chruściel for many valuable comments and suggestions, and for reading a first draft of this article. Supported in part by the Austrian Science Fund (FWF): P 24170-N16.

\section{A Asymptotic solutions of Fuchsian ODEs}

The main object of this appendix is to justify rigorously our use of expansions as asymptotic solutions to Einstein's characteristic constraint equations. Smoothness of these solutions at infinity is a crucial aspect of the analysis, which is why we derive necessary-and-sufficient conditions for the asymptotic expansions to involve no logarithmic terms. To do that we shall proceed as follows: Instead of using $r$ as the independent variable, we introduce $x:=1 / r$ as a new variable and study the transformed ODE near $x=0$. For this we make Taylor expansions of the coefficients appearing in the ODE at $x=0$, and write down the formal polyhomogeneous solutions. The Borel summation lemma guarantees that there exists a function whose polyhomogeneous expansion coincides with the formal series. This function will be shown to approximate the exact solution around $x=0$, from which we eventually conclude that the formal polyhomogeneous solution is in fact an expansion of the exact solution at $x=0$.

To illustrate the procedure, we first show how it works for linear first-order scalar equations in full generality. We then show how this adapts to linear firstorder systems, under conditions corresponding to those that arise in the main text, in order to avoid an uninteresting discussion of several special cases. Every dependence on further variables, which we assume to have compact support, will be suppressed for convenience.

For the definition of polyhomogeneous functions we refer the reader to 7 , Appendix A].

\section{A.1 Formal solutions}

\section{A.1.1 Scalar equation $x \partial_{x} f+h f=g$}

We consider the ODE

$$
x \partial_{x} f+h f=g,
$$

where $x^{-\ell} g=O(1), \ell \in \mathbb{Z}$, and $h=O(1)$ (which clearly includes those cases where $h$ has a zero of any order at $x=0$ ) are assumed to be smooth functions on some interval $\left[0, x_{0}\right)$.

We represent $x^{-\ell} g$ and $h$ via their Taylor expansions at $x=0$,

$$
g \sim \sum_{n=\ell}^{\infty} g_{n} x^{n}, \quad h \sim \sum_{n=0}^{\infty} h_{n} x^{n}
$$

(the symbol $\sim$ has been defined in Section 2.1). We define the indicial exponent to be

$$
\lambda:=-h(0)=-h_{0} .
$$

When considering functions which further depend on angular variables, we will always make the supplementary hypothesis that

$$
h_{0} \text { is angle-independent. }
$$


1st case: $\lambda \notin \mathbb{Z}$. We make the ansatz

$$
f \sim x^{\lambda} \sum_{n=\ell}^{\infty} f_{n+\lambda}^{(0)} x^{n}+\sum_{n=\ell}^{\infty} f_{n}^{(1)} x^{n}=: x^{\lambda} f^{(0)}+f^{(1)} .
$$

Here we use $f^{(a)}$ as short form for the corresponding formal power series (in Section A.2 we shall use Borel summation to obtain a proper function from these formal expansions). In the course of this appendix it will become clear that any solution of (A.1) admits an expansion of the form A.4 , so this ansatz is not restrictive. It follows from (A.1) that $f^{(1)}$ needs to satisfy for any $N \in \mathbb{N}$

$$
\begin{aligned}
& \sum_{n=\ell}^{N} n f_{n}^{(1)} x^{n}+\sum_{n=\ell}^{N} \sum_{k=0}^{n-\ell} h_{k} f_{n-k}^{(1)} x^{n}=\sum_{n=\ell}^{N} g_{n} x^{n}+o\left(x^{N}\right) \\
\Longleftrightarrow \quad & (n-\lambda) f_{n}^{(1)}=g_{n}-\sum_{k=1}^{n-\ell} h_{k} f_{n-k}^{(1)} \text { for } n=\ell, \ell+1, \ldots
\end{aligned}
$$

Since $n-\lambda \neq 0$ by assumption, this defines a unique formal solution $f^{(1)}$ by solving the equations hierarchically. The formal series $f^{(0)}$ needs to satisfy

$$
\begin{gathered}
x^{\lambda} \sum_{n=\ell}^{N}(\lambda+n) f_{n+\lambda}^{(0)} x^{n}+x^{\lambda} \sum_{n=\ell}^{N} \sum_{k=0}^{n-\ell} h_{k} f_{n-k+\lambda}^{(0)} x^{n}=o\left(x^{\lambda+N}\right) \\
\Longleftrightarrow n f_{n+\lambda}^{(0)}=-\sum_{k=1}^{n-\ell} h_{k} f_{n-k+\lambda}^{(0)} \quad \text { for } n=\ell, \ell+1, \ldots
\end{gathered}
$$

We observe that $f_{n+\lambda}^{(0)}=0$ for $n<0$, while $f_{\lambda}^{(0)}$ can be chosen arbitrarily. Once this has been done, A.6 determines the higher-order coefficients. Hence, our ansatz leads to a formal solution, where $f_{\lambda}^{(0)}$ can be thought of as representing the integration constant, or function if angular variables are involved. For convenience we will just speak of an integration constant in what follows.

2nd case: $\lambda \in \mathbb{Z}$. We start with the (again non-restrictive) ansatz

$$
f \sim \sum_{n=\hat{\ell}}^{\infty} f_{n}^{(0)} x^{n}+\log x \sum_{n=\hat{\ell}}^{\infty} f_{n}^{(1)} x^{n}=: f^{(0)}+f^{(1)} \log x,
$$

where we have set $\hat{\ell}:=\min (\lambda, \ell)$. Inserting A.7 into A.1 yields

$$
\begin{gathered}
\log x \quad \sum_{n=\hat{\ell}}^{N} n f_{n}^{(1)} x^{n}+\sum_{n=\hat{\ell}}^{N} f_{n}^{(1)} x^{n}+\log x \sum_{n=\hat{\ell}}^{N} \sum_{k=0}^{n-\hat{\ell}} h_{k} f_{n-k}^{(1)} x^{n}+\sum_{n=\hat{\ell}}^{N} n f_{n}^{(0)} x^{n} \\
+\sum_{n=\hat{\ell}}^{N} \sum_{k=0}^{n-\hat{\ell}} h_{k} f_{n-k}^{(0)} x^{n}=\sum_{n=\hat{\ell}}^{N} g_{n} x^{n}+o\left(x^{N} \log x\right) \\
\quad(n-\lambda) f_{n}^{(1)}+\sum_{k=1}^{n-\hat{\ell}} h_{k} f_{n-k}^{(1)}=0 \\
\text { and } \quad f_{n}^{(1)}+(n-\lambda) f_{n}^{(0)}+\sum_{k=1}^{n-\hat{\ell}} h_{k} f_{n-k}^{(0)}=g_{n} \quad \text { for any } n \geq \hat{\ell} .
\end{gathered}
$$


If $n=\hat{\ell}$, A.8) is understood as $(\hat{\ell}-\lambda) f_{\hat{\ell}}^{(0)}=0$, for $\hat{\ell} \leq n<\lambda$ it leads to $f_{n}^{(1)}=0$. Then (A.9) with $\hat{\ell} \leq n<\lambda$ determines the coefficients $f_{n}^{(0)}$. If $n=\lambda$ (A.8), holds automatically, while (A.9) determines $f_{\lambda}^{(1)}$. The coefficient $f_{\lambda}^{(0)}$ can always be chosen arbitrarily. Once this has been done, all coefficients $f_{n}^{(1)}$ and $f_{n}^{(0)}$ with $\lambda>n$ are determined by the (A.8) and (A.9), respectively. This way we obtain a formal solution with one free parameter, $f_{\lambda}^{(0)}$, which can be regarded as integration constant.

These considerations also reveal that the formal solution (A.7) contains no logarithmic terms if and only if $f_{\lambda}^{(1)}=0$ or, equivalently,

$$
\sum_{k=1}^{\lambda-\hat{\ell}} h_{k} f_{\lambda-k}^{(0)}=g_{\lambda} .
$$

Indeed the vanishing of $f_{\lambda}^{(1)}$ enforces by (A.8) that all the $f_{n}^{(1)}$ 's are zero for $n>\lambda$ as well, while those with $n<\lambda$ have to vanish anyway. Note that (A.10) is in fact a condition relating $h$ and $g$, since the $f_{\lambda-k}^{(0)}$ 's are determined hierarchically from (A.9) (with $f_{n}^{(1)}=0$ ), it does not depend on the boundary conditions as captured by the integration constant.

\section{A.1.2 ODE-system $x \partial_{x} f+h f=g$}

Let us now consider a first-order linear ODE-system,

$$
x \partial_{x} f+h f=g,
$$

where $h=O(1) \in \operatorname{Mat}(n, n)$ and $g=O\left(x^{\ell}\right), \ell \in \mathbb{Z}$. The components of $h$ and $x^{-\ell} g$ are assumed to be smooth functions on the interval $\left[0, x_{0}\right)$. For convenience, and because it suffices for our purposes, we focus on the case $n=2$. Only at some points we add a comment how the general case looks like.

Again, we represent $x^{-\ell} g$ and $h$ via their Taylor expansions at $x=0$,

$$
g \sim \sum_{n=\ell}^{\infty} g_{n} x^{n}, \quad g_{n} \in \mathbb{R}^{2}, \quad h \sim \sum_{n=0}^{\infty} h_{n} x^{n}, \quad h_{n} \in \operatorname{Mat}(2,2) .
$$

There exists a change of basis matrix $T \in \mathrm{GL}(2)$ such that $T h_{0} T^{-1}=: h_{0}^{J}$ adopts Jordan normal form. Hence, it suffices to study the system

$$
x \partial_{x} T f+\left[h_{0}^{J}+\sum_{n=1}^{\infty} T h_{n} T^{-1} x^{n}\right] T f=T g,
$$

or, by relabeling the symbols,

$$
x \partial_{x} f+\left(\sum_{n=0}^{\infty} h_{n} x^{n}\right) f=g,
$$

with either

$$
h_{0}=\left(\begin{array}{cc}
-\lambda_{1} & 0 \\
0 & -\lambda_{2}
\end{array}\right) \quad \text { or } \quad h_{0}=\left(\begin{array}{cc}
-\lambda & 1 \\
0 & -\lambda
\end{array}\right)
$$


We mentioned that a dependence on additional variables is permitted. However, as in the scalar case, we assume

$$
\text { the indicial matrix } h_{0} \text { is angle-independent, }
$$

and thus is a truly constant matrix. In addition, since this covers all cases we are interested in with regard to the main text, we assume that

$$
\lambda, \lambda_{i} \in \mathbb{Z}
$$

1st case: $h_{0}=\left(\begin{array}{cc}-\lambda_{1} & 0 \\ 0 & -\lambda_{2}\end{array}\right)$. W.l.o.g. we assume $\lambda_{1} \leq \lambda_{2}$. Furthermore, we define

$$
\hat{\ell}:=\min \left(\lambda_{1}, \ell\right) .
$$

We make the ansatz (again, later on it will be shown that any solution of A.11) admits an expansion of the form (A.16) )

$$
f_{i} \sim \sum_{k=0}^{2} \log ^{k} x \sum_{n=\hat{\ell}}^{\infty}\left(f_{i}^{(k)}\right)_{n} x^{n}=: f_{i}^{(0)}+f_{i}^{(1)} \log x+f_{i}^{(2)} \log ^{2} x,
$$

where the symbols appearing in this definition are to be understood in the same way as above. The upper index in brackets displays the order of the log term. Whenever useful, the coefficients $f_{k}^{(a)}$ with $k<\hat{\ell}$ are defined as to be zero.

We insert A.16) into (A.12). The coefficients need to satisfy the following set of equations (for $n \geq \hat{\ell}$ ):

(1) $\left(n-\lambda_{i}\right)\left(f_{i}^{(2)}\right)_{n}+F_{i}\left[\left(f^{(2)}\right)_{k}, k<n\right]=0, \quad i=1,2$,

(2) $2\left(f_{i}^{(2)}\right)_{n}+\left(n-\lambda_{i}\right)\left(f_{i}^{(1)}\right)_{n}+G_{i}\left[\left(f^{(1)}\right)_{k}, k<n\right]=0, \quad i=1,2$,

(3) $\left(f_{i}^{(1)}\right)_{n}+\left(n-\lambda_{i}\right)\left(f_{i}^{(0)}\right)_{n}+H_{i}\left[\left(f^{(0)}\right)_{k}, k<n\right]=\left(g_{i}\right)_{n}, \quad i=1,2$,

where $F_{i}, G_{i}$ and $H_{i}$ are multi-linear functions of the indicated quantities. The explicit form of $H_{i}$, which will be needed later on, is

$$
H_{i}=\sum_{k=1}^{n-\hat{\ell}}\left[h_{k}\left(f^{(0)}\right)_{n-k}\right]_{i},
$$

analogously for the other functions. Note that $H_{i}$ generally depends on both components of $f^{(0)}$ since there is no need for the $h_{k}$ 's, $k \geq 1$, to be diagonal.

A solution to the equations (1)-(3) can be constructed as follows: We describe the case $\lambda_{1}<\lambda_{2}$, the case $\lambda_{1}=\lambda_{2}$ can be treated similarly.

$\mathbf{n}<\lambda_{\mathbf{1}}$ : We have to choose $\left(f_{i}^{(2)}\right)_{n}=0$ and $\left(f_{i}^{(1)}\right)_{n}=0$ to fulfill (1) and (2). The coefficients $\left(f_{i}^{(0)}\right)_{n}$ will be generally non-zero and are determined by (3).

$\mathbf{n}=\lambda_{\mathbf{1}}$ : Choose $\left(f_{i}^{(2)}\right)_{\lambda_{1}}=0$ and $\left(f_{2}^{(1)}\right)_{\lambda_{1}}=0$. The first component of (2) (i.e. the one with $i=1$ ) is automatically satisfied, while the first component of (3) determines $\left(f_{1}^{(1)}\right)_{\lambda_{1}}$. The coefficient $\left(f_{1}^{(0)}\right)_{\lambda_{1}}$ is free to choose, while $\left(f_{2}^{(0)}\right)_{\lambda_{1}}$ follows from the second component of (3) (the one with $i=2$ ).

$\lambda_{\mathbf{1}}<\mathbf{n}<\lambda_{\mathbf{2}}$ : (1) still requires $\left(f_{i}^{(2)}\right)_{n}=0$. The coefficients $\left(f_{i}^{(1)}\right)_{n}$ are determined by (2), while the $\left(f_{i}^{(0)}\right)_{n}$ 's are determined by (3). 
$\mathbf{n}=\lambda_{\mathbf{2}}$ : Set $\left(f_{1}^{(2)}\right)_{\lambda_{2}}=0$. The second component of (1) holds automatically, no matter what the value of $\left(f_{2}^{(2)}\right)_{\lambda_{2}}$ is. The coefficient $\left(f_{1}^{(1)}\right)_{\lambda_{2}}$ is determined by the first component of (2). The coefficient $\left(f_{2}^{(2)}\right)_{\lambda_{2}}$ follows from (2). The coefficient $\left(f_{2}^{(1)}\right)_{\lambda_{2}}$ is determined by the second component of (3). The coefficient $\left(f_{1}^{(0)}\right)_{\lambda_{2}}$ follows from (3), while $\left(f_{2}^{(0)}\right)_{\lambda_{2}}$ can be chosen arbitrarily.

$\mathbf{n}>\lambda_{\mathbf{2}}$ : All coefficients $\left(f_{i}^{(j)}\right)_{n}=0$ are determined by (1)-(3).

We remark that for $\lambda_{1}=\lambda_{2}$ the coefficients which can be viewed as integration constants are $\left(f_{i}^{(0)}\right)_{\lambda}, i=1,2$. Moreover, this case implies $f^{(2)}=0$.

Consequently, the ansatz (A.16) leads to a formal solution of A.12 with two free parameters, which can be considered as integration constants $\left(f_{i}^{(0)}\right)_{\lambda_{i}}$.

In fact a similar ansatz, namely

$$
f_{i} \sim \sum_{k=0}^{N} \sum_{n=\hat{\ell}}^{\infty}\left(f_{i}^{(k)}\right)_{n} x^{n} \log ^{k} x,
$$

leads to a formal solution of the corresponding $N$-dimensional system with $h_{0}=$ $\operatorname{diag}\left(-\lambda_{1}, \ldots,-\lambda_{N}\right)$. The integration constants can be identified with $\left(f_{i}^{(0)}\right)_{\lambda_{i}}$.

Logarithmic terms do not appear in (A.16) if and only if

$$
\sum_{k=1}^{\lambda_{i}-\hat{\ell}}\left[h_{k}\left(f^{(0)}\right)_{\lambda_{i}-k}\right]_{i}=\left(g_{i}\right)_{\lambda_{i}}, \quad i=1,2 .
$$

Here one has to distinguish two cases: If

$$
\text { (A.19) is independent of }\left(f_{1}^{(0)}\right)_{\lambda_{1}} \text {, }
$$

then (A.19) is, as in the scalar case, a condition involving exclusively $g$ and $h$, i.e. it just concerns the equations itself and is independent of the boundary conditions. In particular this case occurs for $\lambda_{1}=\lambda_{2}$. Otherwise the appearance of $\log$ terms depends on the value of the integration constant $\left(f_{1}^{(0)}\right)_{\lambda_{1}}$.

2nd case: $h_{0}=\left(\begin{array}{cc}-\lambda & 1 \\ 0 & -\lambda\end{array}\right)$. We use the same ansatz (A.16) as in the 1st case, and with $\hat{\ell}:=\min (\lambda, \ell)$. We insert this ansatz into (A.12) to end up with the following relations among the coefficients $(n \geq \hat{\ell})$ :

(1) $\quad(n-\lambda)\left(f_{i}^{(2)}\right)_{n}+\delta^{1}{ }_{i}\left(f_{2}^{(2)}\right)_{n}+F_{i}\left[\left(f^{(2)}\right)_{k}, k<n\right]=0, i=1,2$,

(2) $2\left(f_{i}^{(2)}\right)_{n}+(n-\lambda)\left(f_{i}^{(1)}\right)_{n}+\delta^{1}{ }_{i}\left(f_{2}^{(1)}\right)_{n}+G_{i}\left[\left(f^{(1)}\right)_{k}, k<n\right]=0, i=1,2$,

(3) $\left(f_{i}^{(1)}\right)_{n}+(n-\lambda)\left(f_{i}^{(0)}\right)_{n}+\delta^{1}{ }_{i}\left(f_{2}^{(0)}\right)_{n}+H_{i}\left[\left(f^{(0)}\right)_{k}, k<n\right]=\left(g_{i}\right)_{n}, i=1,2$,

where $F_{i}, G_{i}$ and $H_{i}$ are again multi-linear functions of the indicated quantities whose explicit formulae look similar to (A.17). We describe how one obtains a solution of these equations:

$\mathbf{n}<\lambda$ : All coefficients are determined by (1)-(3), in particular $\left(f_{i}^{(1)}\right)_{n}=$ $\left(f_{i}^{(2)}\right)_{n}=0$.

$\mathbf{n}=\lambda$ : Equation (1) is fulfilled iff $\left(f_{2}^{(2)}\right)_{\lambda}=0$. The second component of (2) holds since we have chosen $\left(f_{2}^{(2)}\right)_{\lambda}=0$. The first component of (2) enforces 
$2\left(f_{1}^{(2)}\right)_{\lambda}+\left(f_{2}^{(1)}\right)_{\lambda}=0$. In order to satisfy the second component of (3) the yet unspecified $\left(f_{2}^{(1)}\right)_{\lambda}$ (equivalently $\left(f_{1}^{(2)}\right)_{\lambda}$ ) has to be chosen such that

$$
\left(f_{2}^{(1)}\right)_{\lambda}+H_{2}\left[\left(f^{(0)}\right)_{k}, k<\lambda\right]=\left(g_{2}\right)_{\lambda},
$$

whatever is taken for $\left(f_{1}^{(1)}\right)_{\lambda}$ and $\left(f_{2}^{(0)}\right)_{\lambda}$. The first component of (3) can be fulfilled by an appropriate choice of $\left(f_{1}^{(1)}\right)_{\lambda}$, and is independent of $\left(f_{1}^{(0)}\right)_{\lambda}$,

$$
\left(f_{1}^{(1)}\right)_{\lambda}+\left(f_{2}^{(0)}\right)_{\lambda}+H_{1}\left[\left(f^{(0)}\right)_{k}, k<\lambda\right]=\left(g_{1}\right)_{\lambda} .
$$

$\mathbf{n}>\lambda$ : All coefficients $\left(f_{i}^{(j)}\right)_{n}$ are uniquely determined by (1)-(3).

This way we get a formal solution for any choice of $\left(f_{i}^{(0)}\right)_{\lambda}, i=1,2$, which may be regarded as representing the integration constants.

The above algorithm shows that, in the current setting, logarithms are absent if and only if

$$
\left(f_{i}^{(1)}\right)_{\lambda}=0 .
$$

According to A.21), $\left(f_{2}^{(1)}\right)_{\lambda}$ vanishes iff $H_{2}\left[\left(f^{(0)}\right)_{k}, k<\lambda\right]=\left(g_{2}\right)_{\lambda}$, i.e. iff $h$ and $g$ satisfy appropriate relations. However, (A.22) shows that the vanishing of $\left(f_{1}^{(1)}\right)_{\lambda}$ depends on the integration constant $\left(f_{2}^{(0)}\right)_{\lambda}$, i.e. on the boundary conditions, and thus cannot be guaranteed to hold generally. Only specific boundary data lead to solutions without logarithmic terms.

\section{A.2 Borel summation}

We have seen that there exist formal solutions of the ODE $x \partial_{x} f+h f=g$ (in one or two dimensions) of the form

$$
f_{\text {formal }}=\varkappa^{(0)} f^{(0)}+\varkappa^{(1)} f^{(1)}+\varkappa^{(2)} f^{(2)}, \quad \varkappa^{(i)} \in\left\{\log x, \log ^{2} x, x^{\lambda}, 0,1\right\}
$$

where the form of $\varkappa^{(i)}$ depends on the specific value of the indicial exponent $\lambda$. However, in any case all the $f^{(i)}$ 's are formal power series. We can therefore appeal to the Borel Summation Lemma (see, e.g., [4, Appendix D]) which states that for every formal power series $f$ one can find a smooth function $\hat{f}$ whose Taylor expansion, around $x=0$ say, coincides with $f$. Applied to our case that means that there are smooth functions $\hat{f}^{(i)}, i=0,1,2$, such that

$$
\hat{f}:=\varkappa^{(0)} \hat{f}^{(0)}+\varkappa^{(1)} \hat{f}^{(1)}+\varkappa^{(2)} \hat{f}^{(2)} \sim f_{\text {formal }} .
$$

Finally we emphasize that the integration constants are $\left(f^{(0)}\right)_{\lambda}$ in the scalar case, and $\left(f_{i}^{(0)}\right)_{\lambda_{i}}$ or $\left(f_{i}^{(0)}\right)_{\lambda}$, respectively, in the 2-dimensional case, so that the corresponding expansion coefficients of $\hat{f}$ can be specified arbitrarily. This will be crucial for the subsequent argument.

\section{A.3 Approximation of the exact solution}

In the final step we will show that $\hat{f}$ approximates the exact solution $f$ to arbitrary high order in $x$; equivalently, they have the same polyhomogeneous expansions. We denote by $c$ (possibly supplemented by some index) a generic positive constant, while $C$ is supposed to be a constant with a specific value. If 
angular variables are involved $c$ and $C$ will still supposed to be constant. We consider the ODE

$$
x \partial_{x} f+h f=g, \quad h=O(1) \in \operatorname{Mat}(n, n), \quad 0<x<x_{0},
$$

where, as before, the components of $h$ and $x^{-\ell} g$ are smooth functions on $\left[0, x_{0}\right)$. Set

$$
\hat{g}:=x \partial_{x} \hat{f}+h \hat{f} .
$$

By construction of $\hat{f}$ the Taylor expansion of $g-\hat{g}$ at $x=0$ is zero,

$$
\begin{aligned}
& \delta g:=g-\hat{g} \sim 0, \quad \text { i.e. } \\
& \forall N \in \mathbb{N} \exists c^{(N)}>0:\|\delta g\| \leq c^{(N)} x^{N} \forall x<x_{0},
\end{aligned}
$$

where $\|\cdot\|:=\sqrt{\langle\cdot, \cdot\rangle}$. Set $\delta f:=f-\hat{f}$, then

$$
x \partial_{x} \delta f+h \delta f=\delta g .
$$

We want to show that for a given $f$ we can adjust the initial conditions of $\hat{f}$ (equivalently, of the formal solution), such that $\delta f$ is a solution of the ODE and satisfies $\|\delta f\| \leq c^{(N)} x^{N}$ for all $N$. We find

$$
\begin{aligned}
\left|x \partial_{x}\|\delta f\|^{2}\right| & =2\left|\left\langle\delta f, x \partial_{x} \delta f\right\rangle\right|=2|\langle\delta f, \delta g-h \delta f\rangle| \\
& \leq 2|\langle\delta f, \delta g\rangle|+2|\langle\delta f, h \delta f\rangle| \leq(1+2\|h\|)\|\delta f\|^{2}+\|\delta g\|^{2} .
\end{aligned}
$$

Setting $\psi:=\|\delta f\|^{2}$, we thus have

$$
\begin{aligned}
\pm x \partial_{x} \psi & \leq(1+2\|h\|) \psi+\|\delta g\|^{2} \\
\Longrightarrow \quad \pm \partial_{x}\left(\chi^{ \pm 1} \psi\right) & \leq \frac{\|\delta g\|^{2}}{x} \chi^{ \pm 1},
\end{aligned}
$$

where

$$
\chi:=\exp \left(\int_{x}^{x_{0}} \frac{1+2\|h\|}{y} \mathrm{~d} y\right) .
$$

The function $\chi$ satisfies the inequality (with $h_{0} \equiv h(0)$ )

$$
\chi \leq \exp \left(\int_{x}^{x_{0}} \frac{1+2\left\|h_{0}\right\|}{y} \mathrm{~d} y\right) \cdot \underbrace{\exp \left(2 \int_{x}^{x_{0}} \frac{\left\|h-h_{0}\right\|}{y} \mathrm{~d} y\right.}_{=: \hat{\chi}=\mathcal{O}(1)})=x^{-\mu} x_{0}^{\mu} \hat{\chi}
$$

where

$$
\mu:=1+2\left\|h_{0}\right\| \geq 1,
$$

and $\hat{\chi}>0$ is a smooth function bounded away from zero in $\left[0, x_{0}\right)$,

$$
\begin{aligned}
\hat{\chi}^{ \pm 1} & \leq c \\
\Longrightarrow \quad \pm \partial_{x}\left(\chi^{ \pm 1} \psi\right) & \leq c\|\delta g\|^{2} x_{0}^{-\mu} x^{\mu-1} \leq c^{(N)} x^{N} .
\end{aligned}
$$

The inequality with the minus sign yields

$$
\begin{aligned}
& \left(\chi^{-1} \psi\right)(x) \leq\left(\chi^{-1} \psi\right)\left(x_{0}\right)+\int_{x}^{x_{0}} c^{(N)} y^{N} \mathrm{~d} y \leq c \\
& \text { A.31 } \& \text { A.32 '+' } \psi \leq c \chi \leq c x^{-\mu} \\
& \Longrightarrow \quad\|\delta f\| \leq c x^{-\mu / 2} \text {. }
\end{aligned}
$$


We conclude that

$$
\left\|x \partial_{x} \delta f+h_{0} \delta f\right\|=\left\|\delta g-\left(h-h_{0}\right) \delta f\right\| \leq c x^{-\mu / 2+1} .
$$

This leads us to the study of the ODE

$$
x \partial_{x} \tilde{f}+h_{0} \tilde{f}=\tilde{g}
$$

with source $\tilde{g}$ fulfilling $\|\tilde{g}\| \leq c x^{1-\mu / 2}$. The general solution to this equation is

$$
\tilde{f}(x)=x^{-h_{0}} x_{0}^{h_{0}} \tilde{f}\left(x_{0}\right)-x^{-h_{0}} \int_{x}^{x_{0}} y^{h_{0}-1} \tilde{g}(y) \mathrm{d} y .
$$

Here 1 denotes the $n$-dimensional identity matrix. When writing $x^{h_{0}}$ for a matrix $h_{0}$ we mean $\exp \left(h_{0} \log x\right)$. In the following we will distinguish two cases, depending on whether $h_{0}$ can be diagonalized or not.

1st case: We assume that $h_{0}$ can be diagonalized. Clearly this case includes the 1-dim. case. In fact, let us focus for the time being on that case and return to the general case later. Equation (A.37) then implies

$$
\begin{gathered}
|\delta f| \leq c^{(1)} x^{-h_{0}}+c^{(2)} x^{-h_{0}} \int_{x}^{x_{0}} y^{h_{0}-1}\left(|\delta g|+c^{(3)} y|\delta f|\right) \mathrm{d} y \\
\frac{A .34}{\leq} c^{(1)} x^{-h_{0}}+c^{(2)} x^{-h_{0}} \int_{x}^{x_{0}} y^{h_{0}-\mu / 2} \mathrm{~d} y .
\end{gathered}
$$

Replacing $\mu$ by a slightly larger number if necessary, we may assume w.l.o.g. $h_{0}-\mu / 2 \neq-1$. Then

$$
|\delta f| \leq c^{(1)} x^{-h_{0}}+c^{(2)} x^{-h_{0}}\left[\frac{y^{h_{0}-\mu / 2+1}}{h_{0}-\mu / 2+1}\right]_{x}^{x_{0}}=c^{(1)} x^{-h_{0}}+c^{(2)} x^{-\mu / 2+1} .
$$

Suppose that $\mu / 2-1<h_{0}$, then the inequality $|\delta f| \leq c x^{-h_{0}}$ follows. If $\mu / 2-$ $1>h_{0}$, we can merely conclude $|\delta f| \leq c x^{-\mu / 2+1}$. However, this improves the estimate in A.34 by a factor $x$. Repeating the whole procedure $k$-times until $\mu / 2-1-k<h_{0}$ we finally end up with the estimate

$$
\left|x^{h_{0}} \delta f\right| \leq c \text { for all } x \in\left(0, x_{0}\right)
$$

which is independent of the specific relation between $\mu$ and $h_{0}$. Since

$$
\left|\partial_{x}\left(x^{h_{0}} \delta f\right)\right|=x^{h_{0}-1}\left|x \partial_{x} \delta f+h_{0} \delta f\right|=x^{h_{0}-1}\left|\delta g-\left(h-h_{0}\right) \delta f\right| \leq c,
$$

$x^{h_{0}} \delta f$ can be continued to a continuous function on $\left[0, x_{0}\right)$. Hence, multiplying A.37) (with $\tilde{f}=\delta f$ ) by $x^{h_{0}}$, we observe that $\delta F:=x^{h_{0}} \delta f$ is continuous even at $x=0$. Performing the limit $x_{0} \rightarrow 0$, we find

$$
\begin{aligned}
\delta F & =C+\int_{0}^{x} y^{h_{0}-1}\left(\delta g-\left(h-h_{0}\right) \delta f\right) \mathrm{d} y \\
\Longrightarrow \partial_{x} \delta F & =x^{h_{0}-1} \delta g-\frac{h-h_{0}}{x} x^{h_{0}} \delta f
\end{aligned}
$$


for a suitable constant $C$. We read off that the function $\delta F$ is in fact continuously differentiable at $x=0$. Then, by Taylor's theorem,

$$
\begin{aligned}
\delta F & =\left(\partial_{x} \delta F\right)_{0}+O(x)=C+O(x) \\
\Longrightarrow \quad \delta f & =x^{-h_{0}} C+O\left(x^{1-h_{0}}\right) .
\end{aligned}
$$

Recall that in the polyhomogeneous expansion of $\hat{f}$ the coefficient $\hat{f}_{\lambda}^{(0)}$, with $\lambda \equiv-h_{0}$, can be chosen freely. We choose it such that $\delta f_{\lambda} \equiv\left(x^{-\lambda} \delta f\right)_{0}$ vanishes, leading to

$$
\begin{aligned}
\delta f & =x^{-h_{0}} \int_{0}^{x} y^{h_{0}-1}(\delta g-O(y) \delta f) \mathrm{d} y \\
\Longrightarrow \quad|\delta f| & \leq c x^{-h_{0}+1} .
\end{aligned}
$$

Inserting (A.39) into A.38 improves the estimate in each step by a factor of $x$. Repeating this as many times as necessary (there is no disturbing term anymore which is proportional to $x^{-h_{0}}$ and prohibits the improvement of the estimate), we eventually end up with the desired result,

$$
|\delta f| \leq c^{(N)} x^{N} \quad \text { for all } N \text {, i.e. } \quad \delta f \sim 0 .
$$

When dealing with higher-dim. systems we can proceed in a similar manner (note that we have derived the formal solution only for $\lambda_{i} \in \mathbb{Z}$ ). We give a sketch. Denote by $-\lambda_{1}, \ldots,-\lambda_{n}$ the eigenvalues of $h_{0}$ and assume w.l.o.g. $\lambda_{1} \leq \cdots \leq \lambda_{n}$. Equation (A.37) provides the estimate (for $\tilde{g}=\delta g-\left(h-h_{0}\right) \delta f$ )

$$
\left|\delta f_{i}\right| \leq c^{(1)} x^{\lambda_{i}}+c^{(2)} x^{\lambda_{i}} \int_{x}^{x_{0}} y^{-\lambda_{i}-1}\left(\left|\delta g_{i}\right|+c^{(3)} y\|\delta f\|\right) \mathrm{d} y .
$$

Note that the integrand depends on $\|\delta f\|$ and not just on $\left|\delta f_{i}\right|$, because the higher-order terms in the expansion of $h$ do not need to be diagonal.

Again we assume without restriction $\mu / 2+\lambda_{i} \neq 1$. We conclude from A.34 and A.41) that if $\mu / 2-1<-\lambda_{1}$ then $\left|\delta f_{1}\right| \leq c x^{\lambda_{1}}$. Otherwise $\left|\delta f_{i}\right| \leq c x^{-\mu / 2+1}$ for all $i$, and thus $\|\delta f\| \leq c x^{-\mu / 2+1}$. This can be repeated until $\mu / 2-1-k_{1}<$ $-\lambda_{1}$, which means $\left|\delta f_{1}\right| \leq c x^{\lambda_{1}}$. By adjusting the initial conditions via the integration constant $\left(\hat{f}_{1}^{(0)}\right)_{\lambda_{1}}$ appearing in the expansion of $\hat{f}$ one achieves that the $\lambda_{1}$-th order term in the polyhomogeneous expansion of $\delta f_{1}$ vanishes. Then one proceeds in the same way until $\mu / 2-1-k_{2}<-\lambda_{2}$. To continue this process the integration constant $\left(\hat{f}_{2}^{(0)}\right)_{\lambda_{2}}$ has to be chosen suitably. And so on. Eventually one obtains $\|\delta f\| \leq c x^{\lambda_{n}+1}$ and a formula analog to A.38 for higher dimensions. One then straightforwardly establishes the desired estimate,

$$
\|\delta f\| \leq c^{(N)} x^{N} \quad \text { for all } N \text {, i.e. } \quad \delta f \sim 0 .
$$

2nd case: It remains to deal with the case where $h_{0}$ cannot be diagonalized. For reasons of simplicity we restrict attention again to the two-dimensional case. The matrix $h_{0}$ can be brought into Jordan normal form:

$$
h_{0}=\left(\begin{array}{cc}
-\lambda & 1 \\
0 & -\lambda
\end{array}\right) \text {. }
$$


Note that we have derived the formal solution only for $\lambda \in \mathbb{Z}$, which we assume here, as well. First, we compute $x^{h_{0}}$,

$$
x^{h_{0}} \equiv e^{h_{0} \log x} \equiv \sum_{m=0}^{\infty} \frac{h_{0}^{m} \log ^{m} x}{m !}=x^{-\lambda}\left(\begin{array}{cc}
1 & \log x \\
0 & 1
\end{array}\right) .
$$

From equation (A.37) we find

$\delta f(x)=x^{\lambda} x_{0}^{-\lambda}\left(\begin{array}{cc}1 & \log \left(x_{0} / x\right) \\ 0 & 1\end{array}\right) \delta f\left(x_{0}\right)-x^{\lambda} \int_{x}^{x_{0}} y^{-\lambda-1}\left(\begin{array}{cc}1 & \log (y / x) \\ 0 & 1\end{array}\right) \tilde{g}(y) \mathrm{d} y$.

The second component of $\delta f, \delta f_{2}$, fulfills the integral equation

$$
\delta f_{2}(x)=x^{\lambda} x_{0}^{-\lambda} \delta f_{2}\left(x_{0}\right)-x^{\lambda} \int_{x}^{x_{0}} y^{-\lambda-1} \tilde{g}_{2}(y) \mathrm{d} y .
$$

Recall that $\tilde{g}_{i} \equiv \delta g_{i}-\left[\left(h-h_{0}\right) \delta f\right]_{i}$. As before, we may assume w.l.o.g. $\mu / 2-1 \neq$ $-\lambda$, and deduce

$$
\begin{aligned}
\left|\delta f_{2}\right| & \leq c^{(1)} x^{\lambda}+c^{(2)} x^{\lambda} \int_{x}^{x_{0}} y^{-\lambda-1}\left(\left|\delta g_{2}\right|+c^{(3)} y\|\delta f\|\right) \mathrm{d} y \\
& \leq c^{(1)} x^{\lambda}+c^{(2)} x^{-\mu / 2+1}
\end{aligned}
$$

by using (A.34). Next, we consider the first component of (A.44), which, once we have an estimate for $\delta f_{2}$, supplies one for $\delta f_{1}$ :

$$
\begin{aligned}
\delta f_{1}(x)= & x^{\lambda} x_{0}^{-\lambda}\left[\delta f_{1}\left(x_{0}\right)+\log \left(x_{0} / x\right) \delta f_{2}\left(x_{0}\right)\right] \\
& -x^{\lambda} \int_{x}^{x_{0}} y^{-\lambda-1}\left[\tilde{g}_{1}(y)+\tilde{g}_{2}(y) \log (y / x)\right] \mathrm{d} y \\
= & -\delta f_{2}(x) \log x+x^{\lambda} x_{0}^{-\lambda}\left[\delta f_{1}\left(x_{0}\right)+\log x_{0} \delta f_{2}\left(x_{0}\right)\right] \\
& -x^{\lambda} \int_{x}^{x_{0}} y^{-\lambda-1}\left[\tilde{g}_{1}(y)+\tilde{g}_{2}(y) \log y\right] \mathrm{d} y .
\end{aligned}
$$

That yields (w.l.o.g. we assume $x_{0}<1$ )

$$
\left|\delta f_{1}(x)\right| \leq\left|\delta f_{2}(x) \log x\right|+c^{(1)} x^{\lambda}-c^{(2)} x^{\lambda} \int_{x}^{x_{0}}\|\delta f\| y^{-\lambda} \log y \mathrm{~d} y .
$$

The estimate (A.34) supplemented by the one for $\left|\delta f_{2}\right|$, A.46), implies

$$
\begin{aligned}
\left|\delta f_{1}(x)\right| & \leq\left|\delta f_{2} \log x\right|+c^{(1)} x^{\lambda}+\left|x^{\lambda}\left[y^{-\lambda-\mu / 2+1}\left(c^{(2)}+c^{(3)} \log y\right)\right]_{x}^{x_{0}}\right| \\
& \leq c^{(1)} x^{\lambda}|\log x|+c^{(2)} x^{-\mu / 2+1}|\log x| .
\end{aligned}
$$

If $\mu / 2-1<-\lambda$ we are immediately led to $\left|\delta f_{2}(x)\right| \leq c x^{\lambda}$ and $\left|\delta f_{1}(x)\right| \leq$ $c x^{\lambda}|\log x|$, whence $\|\delta f\| \leq c x^{\lambda}|\log x|$.

If the reverse inequality holds, we find $\left|\delta f_{2}(x)\right| \leq c x^{-\mu / 2+1}$ and $\left|\delta f_{1}(x)\right| \leq$ $c x^{-\mu / 2+1}|\log x|$. Combined, that gives $\|\delta f(x)\| \leq c x^{-\mu / 2+1}|\log x|$. Repeating this procedure $k$-times as long as $\mu / 2-k>1-\lambda$ the estimates for $\delta f_{i}$ improve in each round by a factor of $x$, accompanied possibly by the appearance of higher order powers of $\log x$,

$$
\left|\delta f_{2}(x)\right| \leq c x^{-\mu / 2+k}|\log x|^{k-1}, \quad\left|\delta f_{1}(x)\right| \leq c x^{-\mu / 2+k}|\log x|^{k},
$$


with $k$ a positive integer. Anyway, the log terms do not cause any troubles here, because as soon as $k>\mu / 2+\lambda-1$ becomes true, the inequalities

$$
\left|\delta f_{2}\right| \leq c x^{\lambda}, \quad\left|\delta f_{1}\right| \leq c x^{\lambda}|\log x| \quad \Longrightarrow \quad\|\delta f(x)\| \leq c x^{\lambda}|\log x|
$$

replace the estimates with $\mu$. This is owing to the fact that a term of the form $x^{q}, q>0$, kills any $\log$ term, $x^{q} \log ^{m} x \rightarrow 0$ if $x \rightarrow 0$.

We proceed in a similar way as above to show that one can improve the estimates by adjusting the integration constants contained in $\hat{f}$. Recall that

$$
\delta F_{2}(x):=x^{-\lambda} \delta f_{2}(x)=x_{0}^{-\lambda} \delta f_{2}\left(x_{0}\right)-\int_{x}^{x_{0}} y^{-\lambda-1} \tilde{g}_{2}(y) \mathrm{d} y .
$$

From the preceding considerations we conclude that the integrand is $O(\log y)$. Thus we can perform the limit $x \rightarrow 0$. The function $\delta F_{2}$ is continuous on $\left[0, x_{0}\right)$ and we rewrite it as

$$
\delta F_{2}(x)=C+\int_{0}^{x} y^{-\lambda-1} \tilde{g}_{2}(y) \mathrm{d} y=C+O(x|\log x|) .
$$

By choosing the value of $\left(\hat{f}_{2}^{(0)}\right)_{\lambda}$ appropriately (this coefficient was free to choose in our analysis above) one achieves that $C$ vanishes,

$$
\left|\delta f_{2}\right| \leq c x^{\lambda+1}|\log x| .
$$

Combined with $\left|\delta f_{1}\right| \leq c x^{\lambda}|\log x|$, this inequality can be used to improve the estimate for $\left|\delta f_{1}\right|$ : Starting from (A.48) one establishes

$$
\left|\delta f_{1}\right| \leq c x^{\lambda} \stackrel{\stackrel{A .52}{\Longrightarrow}}{\Longrightarrow}\|\delta f\| \leq c x^{\lambda} .
$$

It follows from (A.47) that there exists a constant $C$ with

$$
\lim _{x \rightarrow 0} x^{-\lambda} \delta f_{1}(x)=C,
$$

so one can perform the limit $x_{0} \rightarrow 0$,

$$
\begin{aligned}
x^{-\lambda} \delta f_{1}(x) & =C-x^{-\lambda} \delta f_{2}(x) \log x+\int_{0}^{x} y^{-\lambda-1}\left[\tilde{g}_{1}(y)+\tilde{g}_{2}(y) \log y\right] \mathrm{d} y(A .5) \\
& =C+O(x|\log x|) .
\end{aligned}
$$

We choose the (yet unspecified) value $\left(\hat{f}_{1}^{(0)}\right)_{\lambda}$ in the expansion of $\hat{f}_{1}$ such that $C$ vanishes. Then

$$
\left|\delta f_{1}\right| \leq c x^{\lambda+1}|\log x|
$$

and (A.51) yields

$$
\left|\delta f_{2}\right| \leq c x^{\lambda+1},
$$

i.e. we have improved (A.49) by a factor of $x$.

If we continue this process, an analysis of (cf. A.45) and A.47),

$$
\begin{aligned}
& \delta f_{2}(x)=x^{\lambda} \int_{0}^{x} y^{-\lambda-1} \tilde{g}_{2}(y) \mathrm{d} y \\
& \delta f_{1}(x)=-\delta f_{2}(x) \log x+x^{\lambda} \int_{0}^{x} y^{-\lambda-1}\left[\tilde{g}_{1}(y)+\tilde{g}_{2}(y) \log y\right] \mathrm{d} y
\end{aligned}
$$


reveals that the estimates improve in each step by a factor of $x$, and, since the log term in $\delta f_{1}$ does not matter in the end, we arrive at

$$
\left|\delta f_{i}\right| \leq c^{(N)} x^{N} \quad \text { for all } N \quad \Longleftrightarrow \quad\|\delta f\| \leq c^{(N)} x^{N} \quad \text { for all } N .
$$

Summarizing, we have proved:

Theorem A.1 Consider the linear ODE

$$
x \partial_{x} f+h f=g \quad \text { on } \quad\left(0, x_{0}\right),
$$

where $x^{-\ell} g=O(1), \ell \in \mathbb{Z}$, and $h=O(1)$ are assumed to be smooth maps on $\left[0, x_{0}\right)$, and where $f$ and $g$ have values in $\mathbb{R}$ or $\mathbb{R}^{2}$, with $h$ having values in the space of corresponding linear maps. Let $\hat{f}$ be a solution of the ODE specified by boundary conditions, that is by a choice of the expansion coefficients $\hat{f}_{\lambda}$ in the scalar case, and $\left(\hat{f}_{i}\right)_{\lambda_{i}}$ or $\left(\hat{f}_{i}\right)_{\lambda}$, respectively, in the 2-dimensional case. Denote by $g_{\text {formal }}$ and $h_{\text {formal }}$ the Taylor expansions of $g$ and $h$, respectively, at $x=0$. Then there exists a formal solution $f_{\text {formal }}$ of

$$
x \partial_{x} f+h_{\text {formal }} f=g_{\text {formal }},
$$

such that $f_{\text {formal }}$ is the polyhomogeneous expansion of $\hat{f}$ at $x=0$,

$$
\hat{f} \sim f_{\text {formal }} .
$$

We have further indicated that the theorem remains true in arbitrary dimensions if $h_{0}$ is a diagonal matrix with integer entries.

\section{B Relation between $\kappa=0$ - and $\kappa=\frac{r}{2}|\sigma|^{2}$-gauge}

The metric gauge turned out to be very convenient to construct data for the CFE which are smooth at $\mathscr{I}^{+}$: Global existence and positivity of $\varphi$ and $\nu^{0}$ are implicitly contained in the gauge condition, while the no-logs-condition reduces to a simple algebraic condition on the expansion coefficients of $\gamma$.

Using an affine parameterization, i.e. a $\kappa=0$-gauge, we have to assume that the initial data $[\gamma]$ are chosen in such a way that the function $\varphi$ is positive on $\mathscr{N}$ and that $\varphi_{-1}$ is positive on $S^{2}$. These assumptions do impose geometric restrictions on $\gamma$ in that they exclude data producing conjugate points or even space-time singularities on the initial surface. On the other hand, the gauge choice $\kappa=\frac{r}{2}|\sigma|^{2}$, on a light-cone say, implies that the Raychaudhuri equation admits a global solution with all the required properties without any additional assumptions. To resolve this "paradox" it is useful to understand in which way a $\kappa=\frac{r}{2}|\sigma|^{2}$-gauge is related to other choices of the $r$-coordinate parameterizing the null rays generating the cone, such as affine parameterizations. A similar problem arises for $\nu^{0}$, and can be resolved in the same way.

If the gauge function $\kappa$ depends on the initial data, the physical/geometrical interpretation of the parameter $r$ (its deviation from an affine one) w.r.t. which the $\gamma$ is given, depends on $\gamma$ itself. Due to this "implicit definition" of $r$, the choice $\kappa=\frac{r}{2}|\sigma|^{2}$ conceals geometric restrictions, which we want to discuss now.

We consider initial data $\stackrel{\gamma}{\gamma}$ given in a $\stackrel{\wp}{\kappa}$-gauge on an initial surface $\mathscr{N}$, which we assume for definiteness to be a light-cone, and analyze under which conditions 
a transformation to a $\kappa=\frac{r}{2}|\sigma|^{2}$-gauge is possible, by which we mean that both solutions differ by a coordinate change only. The relevant coordinate transformations $\left(\stackrel{\circ}{r}, \stackrel{\circ}{x}^{A}\right) \mapsto\left(r, x^{A}\right)$ are angle-dependent transformations of the $r$-coordinate

$$
r=r\left(\stackrel{\circ}{r}, \stackrel{\circ}{x}^{A}\right), \quad x^{A}=\stackrel{\circ}{x}^{A} .
$$

It follows from the transformation behavior of connection coefficients that

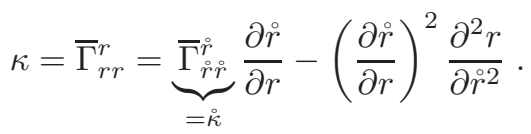

The function $|\sigma|^{2}$ which contains partial derivatives of $r$ (cf. (3.2) ) transforms as

$$
|\sigma|^{2}(r)=\left(\frac{\partial \dot{\circ}}{\partial r}\right)^{2}|\stackrel{\circ}{ }|^{2}(\stackrel{r}{r}(r))
$$

With $\kappa=\frac{r}{2}|\sigma|^{2}$ (B.1) becomes

$$
\frac{\partial^{2} r}{\partial \dot{r}^{2}}-\stackrel{\circ}{\kappa} \frac{\partial r}{\partial \dot{\circ}}+\frac{1}{2} r|\stackrel{\circ}{ }|^{2}=0 \text {. }
$$

We observe that $r\left(\stackrel{\circ}{r}, \stackrel{\circ}{x}^{A}\right)$ and $\stackrel{\circ}{\varphi}\left(\stackrel{\circ}{r}, \stackrel{\circ}{x}^{A}\right)$ satisfy the same ODE. Imposing the boundary conditions $\left.r\right|_{\dot{r}=0}=0$ and $\left.\partial_{\dot{r}} r\right|_{r=0}=1$, we conclude that

$$
r\left(\stackrel{\circ}{r}, \stackrel{\circ}{x}^{A}\right)=\stackrel{\circ}{\varphi}\left(\stackrel{\circ}{r}, \stackrel{\circ}{x}^{A}\right) .
$$

Since $g_{A B}(r)=\stackrel{\circ}{g}_{A B}(r(\stackrel{\circ}{r}))$ the function $\varphi \equiv\left(\frac{\operatorname{det} \check{g}_{\Sigma_{r}}}{\operatorname{det} s}\right)^{1 / 4}$ transforms as a scalar, and

$$
\left.r=\stackrel{\varphi}{(r}\left(r, x^{A}\right), x^{A}\right)=\varphi\left(r, x^{A}\right),
$$

as expected and required by the metric gauge.

To transform from a $\kappa$-gauge to a $\kappa=\frac{r}{2}|\sigma|^{2}$-gauge one simply identifies $\dot{\varphi}$ as the new $r$-coordinate. However, this is only possible when $\left(\stackrel{\circ}{r}, \stackrel{\circ}{x}^{A}\right) \mapsto(r=$ $\stackrel{\circ}{\varphi}, x^{A}=\stackrel{\circ}{x}^{A}$ ) defines a diffeomorphism. Globally this happens if and only if $\dot{\varphi}$ is a strictly increasing function, which is equivalent to the existence of a global solution to the Raychaudhuri equation. Another requirement on $r$ should be that

$$
\lim _{r \rightarrow \infty} r=\infty \quad \Longleftrightarrow \lim _{r \rightarrow \infty} \dot{\varphi}=\infty \quad \Longleftrightarrow \quad \dot{\varphi}_{-1}>0
$$

This derivation clarifies in which way the assumptions on $\dot{\varphi}$ in say a $\stackrel{\kappa}{\kappa}=$ 0-gauge enter: Prescribing smooth data in a $\kappa=\frac{r}{2}|\sigma|^{2}$-gauge one implicitly excludes data violating these assumptions and thereby the existence of conjugate points up-to-and-including conformal infinity. In this work, though, we are only interested in those cases where $\dot{\varphi}$ is strictly increasing with $\dot{\circ}_{-1}>0$, in which case a transition from a $\stackrel{\circ}{\kappa}=0$ - to a $\kappa=\frac{r}{2}|\sigma|^{2}$-gauge is always possible.

Let us take a look at the reversed direction. It follows from (B.1) and the requirement $\left.\partial_{r} \stackrel{r}{r}\right|_{r=0}=1$ that

$$
\frac{r}{2}|\sigma|^{2}=-\left(\frac{\partial \dot{r}}{\partial r}\right)^{2} \frac{\partial^{2} r}{\partial \dot{r}^{2}}=\left(\frac{\partial \dot{\circ}}{\partial r}\right)^{-1} \frac{\partial^{2} \stackrel{\circ}{\partial r^{2}}}{\partial} \frac{\partial \stackrel{\circ}{\partial r}}{\partial e_{0}^{r} \frac{\hat{r}}{2}|\sigma|^{2} \mathrm{~d} \hat{r}}>0,
$$

which defines a diffeomorphism. Consequently, a transformation from a $\kappa=$ $\frac{r}{2}|\sigma|^{2}$-gauge to a $\stackrel{\circ}{\kappa}=0$-gauge is possible without any restrictions.

We conclude that the metric gauge is a reasonable and convenient gauge condition whenever the light-cone is supposed to be globally smooth. 


\section{References}

[1] Y. Choquet-Bruhat, Un théorème d'instabilité pour certaines équations hyperboliques non linéares, C.R. Acad. Sci. Paris 276 (1973), 281-284.

[2] Y. Choquet-Bruhat, P.T. Chruściel, and J.M. Martín-García, The Cauchy problem on a characteristic cone for the Einstein equations in arbitrary dimensions, Ann. Henri Poincaré 12 (2011), 419-482.

[3] P.T. Chruściel, The existence theorem for the general relativistic Cauchy problem on the light-cone, (2012), arXiv:1209.1971 [gr-qc].

[4] P.T. Chruściel, and J. Jezierski, On free general relativistic initial data on the light cone, J. Geom. Phys. 62 (2012) 578-593.

[5] P.T. Chruściel, and M.A.H. MacCallum, D. Singleton: Gravitational Waves in General Relativity. XIV: Bondi Expansions and the "Polyhomogeneity" of Scri, Phil. Trans. Royal Soc. of London A 350, 113-141 (1995).

[6] P.T. Chruściel and T.-T. Paetz, The many ways of the characteristic Cauchy problem, Class. Quantum Grav. 29 (2012), 145006.

[7] P.T. Chruściel, and T.-T. Paetz, Characteristic initial data and smoothness of Scri. I. Framework and results, preprint (2014).

[8] H. Friedrich, Conformal Einstein Evolution, in: The Conformal Structure of Space-Time - Geometry, Analysis, Numerics, J. Frauendiener, H. Friedrich (eds.), Berlin, Heidelberg, Springer, 2002, pp. 1-50.

[9] J. Jezierski, 'Peeling property' for linearized gravity in null coordinates, Class. Quantum Grav. 19 (2002), 2463-2490.

[10] A.D. Rendall, Reduction of the characteristic initial value problem to the Cauchy problem and its applications to the Einstein equations, Proc. Roy. Soc. London A 427 (1990), 221-239.

[11] L.A. Tambourino, and J.H. Winicour, Gravitational fields in finite and conformal Bondi frames, Phys. Rev. 150, 1039-1053 (1966).

[12] R.J. Torrence, and W.E. Couch, Generating fields from data on $\mathscr{H}^{-} \cup \mathscr{H}^{+}$ and $\mathscr{H}^{-} \cup \mathscr{I}^{-}$, Gen. Rel. Grav. 16 (1984), 847-866. 